\title{
Systems of Calogero-Moser Type
}

\author{
S. N. M. Ruijsenaars
}

\begin{abstract}
We survey results on Galilei- and Poincaré-invariant CalogeroMoser and Toda $N$-particle systems, both in the context of classical mechanics and of quantum mechanics. Special attention is given to integrability issues and interconnections between the various models. Action-angle and joint eigenfunction transforms are also considered, and some novel results on $N=2$ eigenfunctions of hyperbolic Askey-Wilson type and of relativistic elliptic type are sketched.
\end{abstract}

\section{Introduction}

This chapter is concerned with a class of finite-dimensional integrable dynamical systems, both at the classical and at the quantum level. The systems model $N$ interacting point particles on a line or a ring. Thus the classical state space is a $2 N$-dimensional symplectic manifold, and the quantum state space a Hilbert space $L^{2}\left(G, d x_{1} \cdots d x_{N}\right)$, with $G$ equal to the classical configuration space.

The systems admit both a nonrelativistic (Galilei-invariant) and a relativistic (Poincaré-invariant) version. The nonrelativistic systems were introduced in the seventies and are known as Calogero-Moser (or CalogeroSutherland) and Toda systems. They can be tied in with the root system $A_{N-1}$ and also admit integrable versions for the remaining root systems. At the classical level all of these systems yield $N$ Poisson commuting independent Hamiltonians with a polynomial dependence on the particle momenta $p_{1}, \ldots, p_{N}$, so that the quantum versions are partial differential operators (PDOs).

The systems just delineated were surveyed in the early eighties by $\mathrm{Ol}$ shanetsky and Perelomov, both in the classical [1] and in the quantium context [2]. These surveys contain extensive lists of references, and are to a large extent concerned with the relations of the systems to group theory, Lie algebra theory, and harmonic analysis on symmetric spaces.

Integrable relativistic generalizations (corresponding once more to the root system $A_{N-1}$ ) were first introduced in Ref. 3 at the classical and in Ref. 4 at the quantum level. The Poisson commuting classical Hamiltoni- 
ans have an exponential dependence on the particle momenta, so that the quantum versions are analytic difference operators (A $\Delta \mathrm{Os})$. The relativistic systems and their relations to the nonrelativistic $A_{N-1}$ systems and various well-known solitonic field theories and spin systems were surveyed in Ref. 5 .

Quite recently, the relativistic systems with pair interactions of the trigonometric type were also shown to admit a generalization to the root systems $B_{N}, C_{N}, D_{N}$, and $B C_{N}$. More precisely, in Ref. 6 van Diejen introduces a quite general integrable quantum system that can be specialized to all of the root systems mentioned above. In further work $[7,8]$ he proposes even more general systems with elliptic interactions, but for these systems integrability has not yet been completely proved. At any rate, the latter systems encompass by specialization virtually all systems of Calogero-Moser and Toda type that are known to be integrable, including external field couplings that go beyond the root system machinery.

We shall deal here exclusively with Galilei- and Poincaré-invariant models. In particular, no external fields and root systems other than $A_{N-1}$ will be treated, and we also omit from consideration thermodynamical aspects, discretizations, $R$-matrix formulations, internal degrees of freedom, and Haldane-Shastry chains. The discussion is addressed principally to theoretical physicists at the graduate student/postdoc level, but we believe it should also be accessible to mathematicians interested in the systems from various viewpoints different from physics. Our emphasis is on expounding the integrability of the systems and their interconnections, and on providing a conceptual understanding of the transforms that diagonalize the commuting dynamics--the action-angle and joint eigenfunction transforms. In doing so, we have tried to use as few ingredients as possible without losing mathematical precision.

We should mention, though, that the models involved can be viewed from a great many angles, and a lot of subfields of mathematics and theoretical physics can be brought to bear on them. Correspondingly, our bare-handed approach will possibly be viewed as a liability rather than an asset by some experts-but these notes are not primarily written for experts. We shall return to various related matters toward the end of this section.

Section 2 is concerned with the nonrelativistic Calogero-Moser and Toda systems in the context of classical mechanics. The mathematics involved in getting a solid grasp of what classical integrability is all about has been particularly well expounded in Refs. 9-11 (in order of increasing sophistication). In Section 2.1 we have summarized some material that can be found (in far greater detail) in these sources. In the process, we introduce various concepts and notation that will reappear later on.

In Section 2.2 we introduce the Calogero-Moser systems. The pair potential characterizing the systems is a rational, hyperbolic, trigonometric, or elliptic function; cf. (2.18)-(2.21) respectively. The former three choices may be viewed as degenerate cases of the latter; cf. (2.22). 
Having in mind readers who are not familiar with elliptic functions, we would like to mention that we use very little of the extensive lore on this subject. Indeed, from a pragmatic viewpoint one may regard (2.63) as a definition of the key function $s\left(x ; \omega, \omega^{\prime}\right)$ in terms of elementary functions. If one now takes (2.64) for granted, and uses (2.50) and (2.52) to introduce the Weierstrass $\mathcal{P}$-function in terms of $s(x)$, then almost all further properties we need readily follow. But if need be, we can highly recommend Ref. 12 to read up on elliptic functions (and, more generally, the classic special functions entering in Section 6.3).

The rational and hyperbolic systems (denoted type I and II, respectively) live on an unambiguous phase space, and each initial state is a scattering state. By contrast, the trigonometric and elliptic systems (denoted type III and IV, respectively) give rise to three distinct phase spaces. The choice of state space depends on whether one views the particles as moving on a line or on a ring, and -in the latter case-on whether one wants to regard the particles as distinguishable or indistinguishable. In all three cases, the internal motion is oscillatory.

In the nonrelativistic case the existence of integrals (conserved quantities) for the defining dynamics is most easily seen via a so-called Lax pair. We sketch the Lax pair formalism in some detail in Section 2.2 and present Lax matrices for each of the four types of pair potentials; cf. (2.32) (type I-III) and (2.51) (type IV). We show how the Lax matrix can be used to deduce that the rational and hyperbolic interactions lead to a scattering of soliton type. That is, the asymptotic momenta are conserved and the position shifts factorize as if independent pair collisions were taking place; cf. (2.47)-(2.48).

In Section 2.3 we introduce the periodic and nonperiodic Toda systems (denoted type V and VI, respectively). In the former, oscillatory motion takes place, whereas the latter lead to soliton scattering. Once more, the integrals can be taken to be power traces or symmetric functions of Lax matrices, given by (2.57) (type V) and (2.59) (type VI). The Toda systems may be viewed as limits of Calogero-Moser systems, as encoded in the connection diagram (2.62). We detail the arrows in this diagram for the various Lax matrices and, therefore, for all of the Poisson commuting integrals at once.

In Section 3 we present and discuss Poincaré-invariant generalizations of the Galilei-invariant systems from Section 2 . Section 3.1 begins by recalling the pertinent space-time symmetry groups and their representation in the classical mechanics description of $N$ free equal mass particles. It is then shown how a natural Ansatz to introduce interactions in the relativistic context can only be made to work provided the "pair potentials" that enter are natural generalizations of the potentials characterizing the CalogeroMoser and Toda systems from Section 2. The structure of the time and space translation generators suggests candidates for Poisson commuting integrals, and these functions (given by (3.19), combined with (3.10), (3.11), 
and (3.15)-(3.17)) are indeed in involution. Thus, integrability follows as a bonus from Poincaré invariance.

In the relativistic case, soliton scattering for the type I, II, and VI systems can be shown without using a Lax matrix; moreover, no Lax matrix is needed to handle the connections in the diagram (2.62)-all of which are detailed in Section 3.1. However, in Section 3.2 we show that the commuting Hamiltonians can be tied in with Lax matrices. These matrices are given by (3.33)-(3.36) (type I-III), (3.41)-(3.44) (type VI), (3.45)-(3.47) (type IV), and (3.53)-(3.58) (type V). Cauchy's identity (3.32) and its elliptic generalization (3.49) are the key to the connection between the Poisson commuting functions (3.19) and the symmetric functions of these matrices.

In the nonrelativistic limit $\beta \rightarrow 0$ (with $\beta=1 / c, c$ denoting the speed of light) the Lax matrices reduce to their counterparts from Section 2, as detailed in (3.38). (Up to diagonal similarity transformations in some cases.) The resulting relation (3.39) between the nonrelativistic and relativistic Hamiltonians yields nonrelativistic integrability as a corollary of the functional equations (3.20) encoding relativistic integrability.

Section 4 deals with the quantum versions of the nonrelativistic and relativistic systems. Section 4.1 has an introductory character. First, we present an algebraic notion of quantum integrability, which is tied to the systems at hand. Specifically, at the nonrelativistic level it amounts to requiring the existence of $N$ commuting independent PDOs, including the defining dynamics. As a PDO the latter is unambiguously determined-in contrast to the $\mathrm{A} \Delta \mathrm{O}$ quantization of the defining relativistic dynamics, which exhibits ordering ambiguities. In the relativistic case, therefore, we speak of an integrable quantization whenever the ordering in the classical Hamiltonians is such that the corresponding quantizations commute as $\mathrm{A} \Delta \mathrm{O}$.

The remainder of Section 4.1 prepares the ground for a reinterpretation (by means of unitary joint eigenfunction transforms) of the commuting PDOs and $A \Delta O$ s as commuting Hilbert space operators. This problem is particularly difficult for the $\mathrm{A} \Delta \mathrm{O}$ s, and only partial solutions have been obtained to date. It is not widely appreciated what is involved here; in fact, even in the commuting PDO case there are no general results ensuring that a unitary joint eigenfunction transform exists. For $A \Delta O$ s this existence problem is greatly aggravated by multiplier ambiguities, as explained in Section 4.1. We have also summarized some of the Hilbert space notions that are essential for a mathematically sound analysis-notions that are, unfortunately, still regarded as outlandish in theoretical physics.

No Hilbert space lore is needed to understand Sections 4.2 and 4.3, however. Here, we address the issue of quantum integrability at the nonrelativistic and relativistic levels, respectively; this issue has an algebraic rather than an analytic character. As it happens, it is actually more straightforward to establish quantum integrability in the relativistic than in the nonrelativistic case. Indeed, with the ordering choice exhibited by (4.35) (type I-IV) and (4.38) (type V and VI) quantum commutativity comes 
down to the functional equations (4.36) and (4.39), respectively. We supplement the direct proofs of nonrelativistic quantum integrability discussed in Section 4.2 with a novel proof at the end of Section 4.3. Here we derive nonrelativistic integrability indirectly, as a corollary of relativistic integrability.

Section 5 is concerned with action-angle transforms-canonical maps $(x, p) \mapsto(\hat{x}, \hat{p})$ that conjugate the Poisson commuting Hamiltonians $H_{k}(x, p)$ to Hamiltonians $H_{k}(\hat{p}), k=1, \ldots, N$. Thus the new Hamiltonians depend only on the actions $\hat{p}_{1}, \ldots, \hat{p}_{N}$. Therefore, they give rise to evolutions of the angles $\hat{x}_{1}, \ldots, \hat{x}_{N}$ that are linear in the respective evolution parameters ("times"). As a result, the commuting dynamics are simultaneously "diagonalized": The action-angle map is the classical analog of the quantum joint eigenfunction transform.

Whenever the commuting Hamiltonians generate complete flows, the Liouville-Arnol'd theorem ensures the existence of action-angle maps on suitable invariant submanifolds of phase space. Unfortunately, this existence theorem is of quite limited practical use, but it does guarantee that one is not wasting time in searching for explicit diagonalizing canonical transformations. The theorem is already sketched at the end of Section 2.1, but for a good understanding of its subject matter it is important to study concrete examples.

We present various elementary examples in Section 5.1. In particular, this enables us to link up action-angle maps and the wave maps from scattering theory, in a very simple setting where phase diagrams can be used. In Section 5.2 we elaborate on this link, showing in particular how the wave map formalism for a large class of repulsive pair potentials entails that all of these potentials give rise to integrable systems. Therefore, it is crucial to single out the systems of type I, II, and VI (for which the wave maps exist on all of phase space) by the extra feature of soliton scattering. (The wave maps are rather well known in quantum mechanics (usually as Møller operators), but not so in classical mechanics. The reader might consult Ref. 10 for the wave map formalism in the latter context. More generally, in Ref. 13 wave maps form the starting point for a great variety of contexts in which scattering takes place, including classical mechanics.)

In Section 5.3 we detail the construction of an action-angle map for the relativistic type II system. This map is, roughly speaking, an interpolation of the incoming and outgoing wave maps. From the construction and its specialization to the nonrelativistic type II system and the type I systems, one readily deduces some highly remarkable duality properties. Specifically, the inverse of the action-angle map serves as an action-angle map for dual systems living on the action-angle phase space, and these dual systems are once more Calogero-Moser systems-as encoded in (5.24). Further spinoffs include a rather explicit picture of an extensive class of evolutions and, as a consequence, a complete elucidation of their long-time asymptotics; cf. $(5.42)-(5.46)$. 
Toward the end of Section 5.3 we also take a brief look at action-angle maps for type III systems. There is much more geometry involved here, since oscillatory motion and partial equilibria are present. On the other hand, the dual systems are once more characterized by a solitonic longtime asymptotics. In this case, however, each of the dual dynamics (of which (5.51) is the simplest representative) gives rise to a codimension-one subvariety containing states that do not have a free asymptotics (due to coinciding velocities, roughly speaking).

In Section 6 we consider eigenfunctions of the PDOs and A $\Delta O$ s at hand, with a bias toward their suitability as kernels of diagonalizing unitaries. This is a subject where many of the key questions are still open. For instance, no eigenfunctions at all are known for the Toda A $\Delta$ Os. Open questions abound for the type II and IV systems, too. Even in the type III case, where transforms in terms of multivariable orthogonal polynomials are known both for the commuting PDOs $[14,15]$ and for the commuting $\mathrm{A} \Delta \mathrm{Os}[16,17]$, the polynomials are not known in a sufficiently explicit way to establish the duality properties expected from the classical level (save for $N=2[5]$ ).

In our survey [5] we have already written on eigenfunctions vs. Hilbert space aspects, and our choice of topics for Section 6 supplements the discussion that can be found there. In particular, we do not reconsider the connections with harmonic analysis on homogeneous spaces associated with classical and quantum groups and algebras-a subject that has mushroomed considerably over the past few years. Instead, we mention a few recent references (among many) that are concerned with this theme from various partly complementary viewpoints, namely Refs. 18-22; further literature can be found there.

Section 6.1 has an introductory character. We specify the PDOs and $\mathrm{A} \Delta \mathrm{Os}$ to be discussed and reappraise the problem of their Hilbert integrability - the question whether and when they can be defined as commuting Hilbert space operators via a unitary joint eigenfunction transform. As simple examples, we present two such transforms for the type I and II PDOs and $\mathrm{A} \triangle \mathrm{Os}$.

In Section 6.2 we sketch how multivariable orthogonal polynomials emerge as joint eigenfunctions for the type III PDOs and $\mathrm{A} \Delta \mathrm{O}$. As we see it, the key idea dates back to Sutherland's paper [14]: All of the operators at issue take a triangular form w.r.t. a suitable partial order on a well-known orthonormal base for the symmetric subspace of $L^{2}\left(\mathbb{T}^{N}\right.$ ) ("free boson eigenstates"), with $\mathbb{T}^{N}$ the $N$-torus. In this case, the multiplier ambiguity for the $\mathrm{A} \Delta \mathrm{O}$ eigenfunctions can be ignored, since any nontrivial multiplier would spoil the polynomial character of the latter. (But when one tries to solve the "band" problem, one needs nonpolynomial eigenfunctions interpolating the polynomials, and so the ambiguity reappears.)

We also use the type III eigenfunctions to illustrate how the nonrelativistic "anyon" particles turn into fermions at the relativistic level. Moreover, 
at the end of Section 6.2 we compare the quantum and classical type III transforms, reading off exactness of semiclassical quantization.

In Section 6.3 we sketch some of our (hitherto unpublished) results on eigenfunctions for the $N=2$ relativistic type II and IV systems. As it turns out, the integral representation for the former which we have found admits a natural generalization to four coupling constants instead of one, and then yields eigenfunctions for the hyperbolic version of the trigonometric Askey-Wilson $\mathrm{A} \Delta \mathrm{O}$ [23]. These novel eigenfunctions have a great many remarkable properties. In particular, they are not only self-dual (in a sense generalizing the self-duality of the relativistic type II system), but they are also simultaneous eigenfunctions for two commuting Askey-Wilson type A $\Delta$ Os acting on the same side of the duality picture.

The Askey-Wilson polynomials can be obtained from these functions by analytic continuation and discretization of one of the two pertinent variables. The self-duality mentioned above already left its footprints for these polynomials, but the second commuting $\mathrm{A} \Delta \mathrm{O}$ for the continuous variable has a trivial action on the polynomials. This is because the latter are periodic, with the period equal to the step size of the relevant $\mathrm{A} \Delta \mathrm{O}$.

The Askey-Wilson polynomials can be tied in with compact quantum groups; more generally, various $q$-special functions correspond to quantum (Hopf) algebras. (See for example Refs. 24-27 and references given there.) It is therefore natural to expect that functions of the type occurring in Section 6.3 are again related to algebraic objects. Candidates include noncompact quantum groups, whose representation theory is still in its infancy.

Section 6.3 is concluded with a description of type IV $N=2$ eigenfunctions corresponding to special coupling constants. The relativistic eigenfunctions generalize the Lamé functions, represented in a form that can be found in Ref. 12. Among other things, we have not yet been able to show that these functions give rise to an orthonormal base for the pertinent Hilbert space, as we do expect. (This is one reason why Ref. 28, already promised in Ref. 5 , has not been published yet.)

We were originally planning an additional section on relations with various well-known infinite-dimensional integrable systems. However, this had to be omitted, so as to keep these lecture notes (and the time spent in writing them) within bounds. Let us, therefore, finish this introduction by just mentioning some of these relations and a few references.

First, as regards the classical versions of the systems, these have already been compared to various soliton field theories and soliton lattices in Ref. 5; cf. also Refs. 3, 29, 30. These infinite-dimensional integrable systems include the Korteweg-de Vries, modified KdV, sine-Gordon, nonlinear Schrödinger, Boussinesq, Hirota-Satsuma and Landau-Lifshitz (XYZ) equations, and the infinite Toda lattice. What emerges from these results is an intimate relation between the $N$-soliton solutions and the $N$-particle relativistic Calogero-Moser systems for special parameter values. More specifically, provided the $N$-soliton scattering maps arising in the infinite sys- 
tems are suitably parametrized, they coincide with the $N$-particle scattering maps of the pertinent Calogero-Moser system. Moreover, for some of the infinite systems (including the $\mathrm{KdV}$, modified $\mathrm{KdV}$, and sine-Gordon equations), the $N$-soliton solutions themselves can be obtained via suitable $N$-particle dynamics, which gives rise to a natural notion of soliton space-time trajectories.

There is meanwhile considerable evidence that the soliton-particle correspondence turns into physical equivalence at the quantum level (i.e., the same scattering and bound-state structure occurs for the quantummechanical particles as for the field- and lattice-theoretic solitons). In particular, it can be shown that the $N=2$ transforms of type II and IV from Section 6.3 have the expected properties on the sine-Gordon and XYZ lines, respectively (cf. also Refs. 5, 31). However, in the absence of explicit $N$-particle relativistic type II and IV eigenfunction transforms with all of the required properties (such as unitarity and factorized scattering), the equivalence remains a conjecture whose plausibility can be disputed.

Finally, we would like to mention a novel theme of more recent vintage than those surveyed in Chapter 4 of Ref. 5. This concerns eigenfunctions of quantum Calogero-Moser models vs. solutions to equations of KnizhnikZamolodchikov type. This relation is currently under active study; most of the relevant literature can be traced from the recent references $[19,20,22$, $32]$.

\section{Classical Nonrelativistic Calogero-Moser and Toda Systems}

\subsection{Background: Classical Mechanics/Symplectic Geometry}

As mentioned previously, the $N$-particle systems at issue describe onedimensional particles. The simplest mathematical description of this physical situation is to let each particle position vary freely over $\mathbb{R}$. An initial state of the system is then encoded in a position vector $x=\left(x_{1}, \ldots, x_{N}\right) \in$ $\mathbb{R}^{N}$ and a momentum vector $p=\left(p_{1}, \ldots, p_{N}\right) \in \mathbb{R}^{N}$, whereas the time evolution is given by Hamilton's equations

$$
\dot{x}_{j}=\frac{\partial H}{\partial p_{j}}, \dot{p}_{j}=-\frac{\partial H}{\partial x_{j}}, \quad j=1, \ldots, N,
$$

with $H(x, p)$ the Hamiltonian (energy function) of the system.

As a first example of this setting, consider a Hamiltonian of the form

$$
H=\sum_{j=1}^{N} \frac{p_{j}^{2}}{2 m_{j}}+U(x)
$$


where $U(x)$ (the potential energy) is a smooth real-valued function on $\mathbb{R}^{N}$. When $U(x)$ vanishes, one is left with the sum of the kinetic energies of $N$ - particles with mass $m_{j}$, and the solution to (2.1) is obviously given by $x_{j}(t)=x_{0, j}+t p_{0, j} / m_{j}, p_{j}(t)=p_{0, j}, j=1, \ldots, N$. Thus, particle $j$ moves with uniform velocity $p_{0, j} / m_{j}$ along the line, without "seeing" the remaining particles. More generally, when $U(x)$ is of the form

$$
U(x)=\sum_{j=1}^{N} V_{j}\left(x_{j}\right)
$$

the particles move independently of each other in external fields. Then the ODE system (2.1) decouples and one is left with solving Newton's ODE $F(y)=m \ddot{y}$, where $F(y)=-V^{\prime}(y)$ is the force field. Since $m \dot{y}^{2} / 2+V(y)$ is time independent, qualitative features of the motion can be read off from a plot of the contour lines $p^{2} / 2 m+V(x)=E$ in the $(x, p)$-plane (phase diagram).

Of most interest for the present lectures is the special case where $U(x)$ is a sum of pair potentials,

$$
U(x)=\sum_{\substack{j, k=1 \\ j<k}}^{N} V_{j k}\left(x_{j}-x_{k}\right) .
$$

This may be viewed as a one-dimensional analog of the three-dimensional gravitational $N$-body problem. In the latter situation the pair potentials $V_{j k}(y), y \in \mathbb{R}^{3}$, are proportional to $1 /|y|$, and so the potential energy diverges when collisions occur. For the Calogero-Moser systems, too, the pair potential is singular at the origin. To avoid such singularities at least for initial states, one should restrict the range of variation of the system position vector $x$. Thus, one chooses initial positions in an open subset $G$ of $\mathbb{R}^{N}$ - the system's configuration space. Then the space of initial states-the system's phase space-is given by the set

$$
\Omega=\left\{(x, p) \in \mathbb{R}^{2 N} \mid x \in G\right\}
$$

As just sketched, the choice of phase space $\Omega$ (the kinematics) depends on the system Hamiltonian $H$ (the dynamics). Assuming from now on that $H$ is a smooth function on $\Omega$, it follows from standard ODE lore that the system (2.1) with initial value $u_{0} \in \Omega$ has a unique solution $u(t) \in \Omega$ for some $t$-interval $\left(-T_{-}, T_{+}\right)$around 0 . But even in the simplest case where $U(x)$ is smooth on $\mathbb{R}^{N}$ and correspondingly one can take $\Omega=\mathbb{R}^{2 N}$, this local solution need not be extendible to a global solution on $(-\infty, \infty)$ : one or more of the particles may escape to infinity in finite time. In the "next simplest" case where a collision set is discarded, it can also happen that collisions do occur after a finite time. 
The first question to answer, therefore, is whether for a given $u_{0} \in \Omega \mathbf{a}$ global solution $u(t) \in \Omega, t \in \mathbb{R}$, exists. If this is the case, one can ask questions about the long-time characteristics of the trajectory, such as whether it stays in bounded subsets of $\Omega$ (corresponding to an $N$-body bound state), or whether it exhibits a free motion

$$
u(t) \sim\left(x^{ \pm}+t v^{ \pm}, p^{ \pm}\right), \quad t \rightarrow \pm \infty,
$$

for asymptotic times (corresponding to a scattering state).

Of course, such questions can be more easily dealt with when one is able to solve Hamilton's equations (2.1) in a sufficiently explicit way. But this appears impossibly difficult for most Hamiltonians of physical interest, and certainly so for the $N$-body gravitational Hamiltonian. Accordingly, the latter context gives rise to simple qualitative questions that are wide open even three centuries after Newton.

The Calogero-Moser and Toda Hamiltonians are notable exceptions to this rule. An important property of these Hamiltonians is that they are integrable - a notion we shall discuss shortly. First, however, we would like to recall some geometric formalism that makes it possible to handle (finitedimensional) classical mechanics in a mathematically precise and concise way.

As a quite general-state space on which Hamiltonian mechanics can be defined, one can take a $2 N$-dimensional differentiable manifold $\Omega$ equipped with a nondegenerate closed 2 -form $\omega$-a symplectic manifold $\langle\Omega, \omega\rangle$. The cotangent bundle of an $N$-dimensional differentiable manifold $G$ can be equipped with such a form in a natural way, and we shall mostly specialize to this setting. In particular, viewing the phase space (2.2) as the cotangent bundle to the open set $G \subset \mathbb{R}^{N}$, this symplectic form reads

$$
\omega=\sum_{j=1}^{N} d x_{j} \wedge d p_{j} .
$$

More generally, fixing a point $u_{0}$ in a symplectic manifold $\langle\Omega, \omega\rangle$, there exist coordinates $(x(u), p(u)) \in \mathbb{R}^{2 N}$ for $u$ in a neighborhood $\mathcal{U}$ of $u_{0}$ such that $\omega$ takes the form (2.3) on $\mathcal{U}$ (Darboux's theorem); such coordinates are referred to as canonical (or symplectic) coordinates.

Since $\omega$ is nondegenerate, a 1 -form $\alpha$ on $\Omega$ gives rise to a vector field $X^{(\alpha)}$ on $\Omega$, uniquely determined by requiring

$$
\omega\left(X^{(\alpha)}, X\right)=\alpha(X)
$$

for arbitrary vector fields $X$. To obtain a dynamics on the symplectic manifold $\Omega$ one can now start from any real-valued smooth function $H$ on $\Omega$ and introduce the associated Hamiltonian vector field $X_{H} \equiv X^{(d H)}$; then the time evolution is governed by

$$
\dot{u}=X_{H}(u), \quad u \in \Omega
$$


and the corresponding flow $\Omega \rightarrow \Omega, u_{0} \mapsto u(t)$ is written $\exp \left(t X_{H}\right)$, or briefly $e^{t H}$. As before, this flow is a priori only locally defined, with the time interval depending on $u_{0}$. When all trajectories stay in $\Omega$ for all times, the flow is called complete. Showing completeness for Hamiltonians of physical interest may be quite difficult. In purely mathematical work, however, one often assumes that $\Omega$ is compact. Then the completeness problem evaporates, since in that case any Hamiltonian flow is complete.

In the special (noncompact) setting (2.2), (2.3), we have $H=H(x, p)$ and

$$
\begin{aligned}
& d H=\sum_{j=1}^{N}\left(\frac{\partial H}{\partial x_{j}} d x_{j}+\frac{\partial H}{\partial p_{j}} d p_{j}\right) \\
& X_{H}=\sum_{j=1}^{N}\left(\frac{\partial H}{\partial p_{j}} \frac{\partial}{\partial x_{j}}-\frac{\partial H}{\partial x_{j}} \frac{\partial}{\partial p_{j}}\right) .
\end{aligned}
$$

Thus, (2.4) can be rewritten as

$$
(\dot{x}, \dot{p})^{t}=\mathcal{S} \nabla H, \quad \mathcal{S} \equiv\left(\begin{array}{cc}
0 & 1_{N} \\
-1_{N} & 0
\end{array}\right),
$$

which amounts to (2.1).

Returning to the general case, we introduce the Poisson bracket

$$
\{\cdot, \cdot\}: C^{\infty}(\Omega) \times C^{\infty}(\Omega) \rightarrow C^{\infty}(\Omega), \quad(F, G) \mapsto\{F, G\} \equiv \omega\left(X_{F}, X_{G}\right) .
$$

(In Sections 2, 3, and 5, we use the symbol $C^{\infty}(M)$ to denote the space of real-valued smooth functions on $M$.) Recalling the above definition of Hamiltonian vector fields, this can also be written

$$
\{F, G\}=d F\left(X_{G}\right)=-d G\left(X_{F}\right)=-X_{F}(G)=X_{G}(F) .
$$

It is easily checked that the Poisson bracket equips $C^{\infty}(\Omega)$ with a Lie algebra structure: $\{\cdot, \cdot\}$ is bilinear, antisymmetric, and satisfies the Jacobi identity. The relation to the Lie bracket $[\cdot, \cdot]$ (anticommutator) on the Hamiltonian vector fields is given by

$$
\left[X_{F}, X_{G}\right]=X_{-\{F, G\}}
$$

In the special case (2.2), (2.3) one can write, more concretely,

$$
\{F, G\}=\sum_{j=1}^{N}\left(\partial_{x_{j}} F \partial_{p_{j}} G-\partial_{p_{j}} F \partial_{x_{j}} G\right)=\nabla F \cdot S \nabla G
$$

whence one obtains the canonical commutation relations

$$
\left\{x_{i}, x_{j}\right\}=\left\{p_{i}, p_{j}\right\}=0, \quad\left\{x_{i}, p_{j}\right\}=\delta_{i j}, \quad i, j=1, \ldots, N .
$$


Fixing a Hamiltonian $H \in C^{\infty}(\Omega)$, one can now characterize the functions $\mathcal{I}_{H} \subset C^{\infty}(\Omega)$ that are conserved under the $H$ flow $e^{t H}$-the so-called integrals: One has

$$
I \in \mathcal{I}_{H} \Longleftrightarrow\{I, H\}=0 \text {. }
$$

Indeed, this follows from

$$
\frac{d I}{d t}=X_{H}(I)=\{I, H\}
$$

where the argument $\exp \left(t X_{H}\right)\left(u_{0}\right)$ is suppressed. From antisymmetry it is immediate that $H \in \mathcal{I}_{H}$. More generally, assuming $I_{1}, \ldots, I_{k} \in \mathcal{I}_{H}$, any $f \in C^{\infty}\left(\mathbb{R}^{k}\right)$ gives rise to a function $f\left(I_{1}, \ldots, I_{k}\right) \in \mathcal{I}_{H}$, as is easily verified. Thus, $\mathcal{I}_{H}$ is an (infinite-dimensional) algebra. Using the Jacobi identity one infers that $\mathcal{I}_{H}$ is also a Lie algebra w.r.t. the Poisson bracket.

We proceed by discussing symplectic maps. First, let us assume that $\Omega$ is an open subset of $\mathbb{R}^{2 N}$ with coordinates $(x, p)$ and symplectic form (2.3). A diffeomorphism

$$
\Phi: \Omega \rightarrow \widehat{\Omega}, \quad(x, p) \mapsto(\hat{x}, \hat{p}),
$$

onto an open subset $\widehat{\Omega}$ of $\mathbb{R}^{2 N}$ is then called a canonical transformation when the functions $\hat{x}_{1}(x, p), \ldots, \hat{p}_{N}(x, p) \in C^{\infty}(\Omega)$ satisfy the canonical commutation relations

$$
\left\{\hat{x}_{i}, \hat{x}_{j}\right\}=\left\{\hat{p}_{i}, \hat{p}_{j}\right\}=0,\left\{\hat{x}_{i}, \hat{p}_{j}\right\}=\delta_{i j}, \quad i, j=1, \ldots, N .
$$

Equivalently, for any $(x, p) \in \Omega$ the Jacobian matrix $(D \Phi)(x, p)$ belongs to the symplectic group $\operatorname{Sp}(2 N, \mathbb{R})$, i.e.,

$$
(D \Phi) \mathcal{S}(D \Phi)^{t}=\mathcal{S} .
$$

A third equivalent definition can be used to introduce a coordinate-free generalization. To state this definition, we equip $\widehat{\Omega}$ with the symplectic form

$$
\widehat{\omega} \equiv \sum_{j=1}^{N} d \hat{x}_{j} \wedge d \hat{p}_{j} .
$$

Then the above map (2.8) is a canonical transformation iff

$$
\Phi^{*} \widehat{\omega}=\omega
$$

A symplectic map (or symplectomorphism) between two symplectic manifolds $\langle\Omega, \omega\rangle$ and $\langle\widehat{\Omega}, \widehat{\omega}\rangle$ is now a diffeomorphism $\Phi$ from $\Omega$ onto $\widehat{\Omega}$ such that 
(2.10) holds true. Equivalently, one can require that $\Phi$ preserve Poisson brackets. That is, letting

$$
F \equiv \Phi^{*} \widehat{F}, \quad \widehat{F} \in C^{\infty}(\widehat{\Omega}),
$$

one should have

$$
\{F, G\}=\{\widehat{F}, \widehat{G}\}, \quad \forall \widehat{F}, \widehat{G} \in C^{\infty}(\widehat{\Omega}) .
$$

A complete Hamiltonian flow is readily shown to yield a 1-parameter group of symplectic maps $\exp (t H):\langle\Omega, \omega\rangle \rightarrow\langle\Omega, \omega\rangle$.

For canonical transformations one often interprets the functions $\hat{x}(x, p)$ and $\hat{p}(x, p)$ as new coordinates on $\Omega$, which have the special property that the symplectic form $\omega$ given by (2.3) can be written as $\sum_{j} d \hat{x}_{j} \wedge d \hat{p}_{j}$; Thus, these coordinates are canonical, just as the coordinates $x, p$; cf. the definition below (2.3). The key property of canonical transformations is that they leave Hamilton's equations invariant. That is, setting

$$
\widehat{H}(\hat{u}) \equiv H(\mathcal{E}(\hat{u})), \quad \mathcal{E} \equiv \Phi^{-1}, \hat{u} \equiv(\hat{x}, \hat{p}),
$$

and assuming $\hat{u}(t)$ solves the Hamilton equations

$$
\frac{d \hat{u}}{d t}=\mathcal{S} \nabla_{\hat{u}} \widehat{H}(\hat{u})
$$

in the new coordinates, one gets a solution $u(t) \equiv \mathcal{E}(\hat{u}(t))$ to the Hamilton equations

$$
\frac{d u}{d t}=\mathcal{S} \nabla_{u} H(u), \quad u=(x, p),
$$

in the original coordinates. (Indeed, this follows from the chain rule and the canonicity property (2.9).) Reformulated in the coordinate-free setting of symplectic maps, this state of affairs amounts to a commutative diagram

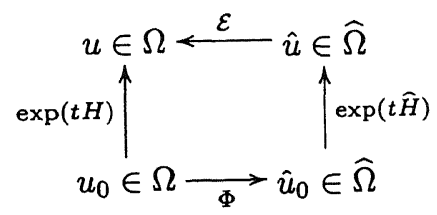

i.e., to the equality

$$
e^{t H}=\mathcal{E} \circ e^{t \widehat{H}} \circ \Phi, \quad \mathcal{E} \equiv \Phi^{-1}, \widehat{H} \equiv \mathcal{E}^{*}(H) .
$$

We are now prepared to discuss the notion of integrability. Fixing $H \in$ $C^{\infty}(\Omega)$, one calls $H$ an integrable Hamiltonian when there exist integrals $I_{1}=H, I_{2}, \ldots, I_{N}$ that are independent and in involution. That is, their 
gradients $d I_{1}, \ldots, d I_{N}$ are linearly independent on an open dense subset of $\Omega$ and they mutually (Poisson) commute.

Let us first illustrate this definition with a trivial, yet instructive example. Consider the Hamiltonian $H(x, p) \equiv p_{1}$ on the phase space (2.2). Then any $I \in C^{\infty}(\Omega)$ that does not depend on $x_{1}$ belongs to $\mathcal{I}_{H}$. In particular, $H$ is integrable in the above sense, since one can choose, for instance, $I_{k}$ equal to $x_{k}$ or $p_{k}$ for $k=2, \ldots, N$. Each of these $2^{N-1}$ choices leads to distinct maximal Abelian subalgebras of $\mathcal{I}_{H}$ (which is itself non-Abelian), consisting of functions $f\left(I_{1}, \ldots, I_{N}\right), f \in C^{\infty}\left(\mathbb{R}^{N}\right)$. (As is readily seen, a symplectic form cannot vanish on a $k$-dimensional subspace for $k>N$; this is why the subalgebras cannot be enlarged without violating commutativity.)

The definition of integrability just exemplified can be widely found in the physics literature. It is however not strong enough to guarantee the applicability of the Liouville-Arnol'd theorem. Moreover, it does not single out Calogero-Moser Hamiltonians among $N$-particle Hamiltonians of the form

$$
H=\frac{1}{2} \sum_{j=1}^{N} p_{j}^{2}+g^{2} \sum_{\substack{j, k=1 \\ 1 j<k}}^{N} V\left(x_{j}-x_{k}\right), \quad g>0
$$

with a repulsive pair potential $V(x)$, since any such Hamiltonian is integrable. In this subsection we do not elaborate on the latter assertion (this is deferred to Section 5.2), but we do want to discuss the Liouville-Arnol'd theorem. Accordingly, we should first sharpen the above definition of integrability.

We shall henceforth call a Hamiltonian Liouville integrable iff (i) it is integrable in the above sense; (ii) the flows generated by $I_{1}, \ldots, I_{N}$ are complete. To appreciate the additional restriction, it is important to be aware of the fact that any Hamiltonian $H$ on a symplectic manifold $\langle\Omega, \omega\rangle$ is integrable in a neighborhood $\mathcal{U}$ of any point $u_{0} \in \Omega$ for which $d H\left(u_{0}\right) \neq 0$. Indeed, it can be proved that there exist canonical coordinates $x(u), p(u)$ on $\mathcal{U}$ such that $H(u)=p_{1}(u)$ on $\mathcal{U}$; therefore, the example just discussed applies. Of course, the crux is that typically $\mathcal{U}$ will not be complete under the flows $\exp \left(t_{j} I_{j}\right)$.

Let us now assume that a given $H \in C^{\infty}(\Omega)$ is Liouville integrable. Fixing a point $u_{0} \in \Omega$ at which the gradients $d I_{1}, \ldots, d I_{N}$ are linearly independent, consider

$$
M\left(u_{0}\right) \equiv\left\{u(t) \mid t \in \mathbb{R}^{N}\right\}, \quad u(t) \equiv \exp \left(t_{1} I_{1}\right) \cdots \exp \left(t_{N} I_{N}\right)\left(u_{0}\right) .
$$

This is a well-defined subset of $\Omega$, since the flows are complete on $\Omega$. Moreover, linear independence of the gradients entails that $M\left(u_{0}\right)$ is an $N$-dimensional submanifold. Since the flows commute, the set $\left\{t \in \mathbb{R}^{N}\right.$ $\left.u(t)=u_{0}\right\}$ is a discrete subgroup of $\mathbb{R}^{N}$. From this one infers that $M\left(u_{0}\right)$ 
is diffeomorphic to $\mathbb{T}^{k} \times \mathbb{R}^{N-k}$ for some $k \in\{0,1, \ldots, N\}$. (In particular, when $M\left(u_{0}\right)$ is compact, one must have $k=N$.)

These somewhat sketchy remarks are the starting point for the LiouvilleArnol' $^{d}$ theorem. This theorem asserts that on suitable disjoint, open and connected submanifolds $\Omega_{i}, i=1,2, \ldots$ there exist canonical coordinates $x(u), p(u)$ such that the commuting Hamiltonians $I_{1}=H, I_{2}, \ldots, I_{N}$ depend solely on $p_{1}, \ldots, p_{N}$ (the so-called action variables), whereas the angle variables $x_{1}, \ldots, x_{N}$ vary over $\mathbb{R}$ or the torus $\mathbb{T}^{1}$. Thus the flow $\exp \left(t_{j} I_{j}\right), j \in\{1, \ldots, N\}$, amounts to a translation of the angle variables that is linear in the evolution parameter $t_{j}$.

Of course, our description of this theorem is incomplete as long as we do not define the qualifier "suitable." We shall have more to say about this in Section 5. For the time being we mention that "suitable" includes first of all the assumption that the gradients $d I_{1}, \ldots, d I_{N}$ are linearly independent on $\Omega_{i}$ and that the commuting flows are complete on $\Omega_{i}$. This assumption already entails that $\Omega_{i}$ is a union of $N$-dimensional submanifolds of the form (2.14). As a second assumption, these submanifolds of $\Omega_{i}$ should all be diffeomorphic.

For our purposes it is convenient to reinterpret the existence of actionangle coordinates on the submanifold $\Omega_{i}$ as the existence of a symplectic map

$$
\Phi_{i}:\left\langle\Omega_{i}, \omega\right\rangle \rightarrow\left\langle\widehat{\Omega}_{i}, \hat{\omega}_{i}\right\rangle, \quad u \mapsto(\hat{x}, \hat{p})
$$

onto a new manifold of the form

$$
\widehat{\Omega}_{i}=M_{i} \times A_{i}
$$

with

$$
M_{i} \equiv \mathbb{T}^{k_{i}} \times \mathbb{R}^{N-k_{i}}, \quad k_{i} \in\{0,1, \ldots, N\},
$$

and $A_{i}$ an open connected subset of $\mathbb{R}^{N}$; the symplectic form on $\widehat{\Omega}_{i}$ reads

$$
\widehat{\omega}_{i}=\sum_{j=1}^{N} d \hat{x}_{j} \wedge d \hat{p}_{j} .
$$

Here, $\hat{p}$ varies over $A_{i}, \hat{x}_{1}, \ldots, \hat{x}_{k_{i}}$ over $(-\pi, \pi]$ (so $\mathbb{T}^{1}$ is viewed as $\mathbb{R} / 2 \pi \mathbb{Z}$ ) and $\hat{x}_{k_{i}+1}, \ldots, x_{N}$ over $\mathbb{R}$. This reinterpretation is notationally and conceptually useful, since our starting point differs considerably from the abstract, coordinate-free setting of the theorem. Indeed, our Hamiltonian $H$ is typically given as a concrete function of canonical coordinates $x, p$, whose range of variation serves to define $\Omega$. Whenever one can show that $H(x, p)$ is Liouville integrable, one should try and concretize the submanifolds $\Omega_{i}$ and action-angle maps $\Phi_{i}$, and obtain in particular explicit functions of $\hat{p}$ for the transformed integrals. In Section 5 we shall illustrate this general program with several concrete examples. 


\subsection{Calogero-Moser Systems}

The Calogero-Moser systems are dynamical systems defined by $N$-particle Hamiltonians of the form (2.13) with a special choice of pair potential $V(x)$. One can distinguish four different types, denoted by I-IV:

I.

II.

III.

IV.

Here, $\mathcal{P}$ is the Weierstrass function, a doubly periodic meromorphic function with primitive periods $2 \omega, 2 \omega^{\prime}$ and double poles at the period lattice. The type I-III systems can be viewed as limiting cases of the type IV system, since one has

$$
\mathcal{P}\left(x ; \omega, \omega^{\prime}\right)= \begin{cases}1 / x^{2}, & \omega=\infty, \omega^{\prime}=i \infty \\ \nu^{2} / 3+\nu^{2} / \operatorname{sh}^{2} \nu x, & \omega=\infty, \omega^{\prime}=i \pi / 2 \nu \\ -\nu^{2} / 3+\nu^{2} / \sin ^{2} \nu x, & \omega=\pi / 2 \nu, \omega^{\prime}=i \infty\end{cases}
$$

Consider first the type I and II cases. Discarding the collision sets $x_{i}=x_{j}$ from $\mathbb{R}^{N}$, one obtains an open set $\mathbb{R}_{\neq}^{N}$ with $N$ ! connected components, corresponding to the various particle orderings. On $\mathbb{R}_{\neq}^{N}$ the potential energy is a smooth function. Fixing an initial point $\left(x_{0}, p_{0}\right) \in \mathbb{R}_{\neq}^{N} \times \mathbb{R}^{N}$, the energy $E_{0}=H\left(x_{0}, p_{0}\right)$ is conserved along the corresponding orbit $(x(t), p(t))$. Since the potential is positive, this leads to an upper bound on $|p(t)|$ and a nonzero lower bound on the particle distances. Therefore, no collisions can occur, and escape to infinity in finite time is excluded, since $\left|\dot{x}_{j}(t)\right|=$ $\left|p_{j}(t)\right| \leq C$ along the orbit. Hence the flow is complete, and we may as well restrict attention to the phase space

$$
\Omega \equiv G \times \mathbb{R}^{N}, \quad G \equiv\left\{x \in \mathbb{R}^{N} \mid x_{N}<\cdots<x_{1}\right\},
$$

with its canonical form (2.3).

Next, consider the system of type III, often called the Sutherland system. In this case one can distinguish three different versions of the Hamiltonian. First, one can again avoid the singularities of the potential by deleting the sets $x_{i} \equiv x_{j}(\bmod 2 \pi / \mu)$ from $\mathbb{R}^{N}$. This yields an infinite number of connected components, each giving rise to phase spaces on which the Hamiltonian flow is complete. (This follows in the same way as before from energy conservation.) We restrict attention to one of these,

$$
\widetilde{\Omega} \equiv G \times \mathbb{R}^{N}, \quad G \equiv\left\{\begin{array}{l|l}
x \in \mathbb{R}^{N} & \begin{array}{c}
x_{N}<\cdots<x_{1} \\
x_{1}-x_{N}<2 \pi / \mu
\end{array}
\end{array}\right\}
$$


This choice amounts to a fixed ordering and minimal distances between the particles. We equip $\widetilde{\Omega}$ with the form (2.3), and so obtain once more a symplectic manifold of the cotangent bundle type.

The choice of phase space just delineated is mathematically acceptable, but appears unnatural from a physical standpoint. One would rather like to view the $x_{j}$ 's as encoding angular positions, so that the type III Hamiltonian describes $N$ particles on a ring that interact via an $1 / d^{2}$ potential, where $d$ is the distance between particles as measured in the plane of the ring.

This interpretation can be mathematically implemented as follows. Consider the map

$$
\begin{aligned}
& \Gamma:\left(x_{1}, \ldots, x_{N}, p_{1}, \ldots, p_{N}\right) \\
& \mapsto\left(x_{N}+\frac{2 \pi}{\mu}, x_{1}, \ldots, x_{N-1}, p_{N}, p_{1}, \ldots, p_{N-1}\right) .
\end{aligned}
$$

Restricted to $\widetilde{\Omega}$, this map is bijective, has no fixed points, and is symplectic. As such, it generates a symplectic $\mathbb{Z}$-action on $\widetilde{\Omega}$, and we may divide out this action to obtain a new symplectic manifold

$$
\Omega \equiv \widetilde{\Omega} / \mathbb{Z}
$$

equipped with the quotient form, again denoted by $\omega$.

A simple choice of coordinates for $\Omega$ reads

$$
\Omega \simeq\left\{(x, p) \in \mathbb{R}^{2 N} \mid x \in F_{N}\right\}
$$

where $F_{N}$ is defined by

$$
F_{N} \equiv\left\{x \in \mathbb{R}^{N} \mid-\frac{\pi}{\mu}<x_{N}<\cdots<x_{1} \leq \frac{\pi}{\mu}\right\} .
$$

This choice is in accordance with the above-mentioned physical picture of particles occupying distinct positions on a ring, with the particles viewed as indistinguishable. Indeed, an initial state of this physical system can be uniquely encoded in $N$ phase factors $\exp \left(i x_{j} / \mu\right), j=1, \ldots, N, x \in F_{N}$, regarded as positions on the ring $S^{1} \subset \mathbb{C} \simeq \mathbb{R}^{2}$, and in associated momenta $p_{1}, \ldots, p_{N} \in \mathbb{R}$.

From a mathematical point of view the choice (2.26) and coordinatization (2.27) are also natural. Indeed, the type III Hamiltonian on $\tilde{\Omega}$ is invariant under the $\mathbb{Z}$-action generated by $\Gamma$ (i.e., it takes the same values on orbits of $\Gamma)$. Thus, its (complete) flow on $\widetilde{\Omega}$ descends to a well-defined and complete flow on $\Omega$. Moreover, $F_{N} \times \mathbb{R}^{N}$ is a fundamental set for the $\mathbb{Z}$-action on $\widetilde{\Omega}$. That is, for a given $(\tilde{x}, \tilde{p}) \in \widetilde{\Omega}$, there exist uniquely determined $x \in F_{N}$, $l \in\{1, \ldots, N\}$ and $m \in \mathbb{Z}$ such that

$$
x_{1}=\tilde{x}_{l}+\frac{2 \pi m}{\mu}
$$




$$
\begin{gathered}
\vdots \\
x_{N-l+1}=\tilde{x}_{N}+\frac{2 \pi m}{\mu} \\
x_{N-l+2}=\tilde{x}_{1}+\frac{2 \pi(m-1)}{\mu} \\
\vdots \\
x_{N}=\tilde{x}_{l-1}+\frac{2 \pi(m-1)}{\mu}
\end{gathered}
$$

and then $p$ is given by

$$
\begin{gathered}
p_{1}=\tilde{p}_{l} \\
\vdots \\
p_{N-l+1}=\tilde{p}_{N} \\
p_{N-l+2}=\tilde{p}_{1} \\
\vdots \\
p_{N}=\tilde{p}_{l-1} .
\end{gathered}
$$

At this point it should be emphasized that the coordinatization (2.27), (2.28) is a set-theoretic one: each $\Gamma$-orbit in $\widetilde{\Omega}$ is labeled by a unique $(x, p) \in$ $F_{N} \times \mathbb{R}^{N}$. There are no globally defined smooth coordinates on $\Omega$, just as no global smooth coordinate function exists on $\mathbb{T}^{1}$. Of course, on the open dense coordinate patch $\left\{x_{1}<\pi / \mu\right\}$ the coordinates are smooth, and on this patch the quotient form is given by (2.3). However, one cannot simply discard the subset $\left\{x_{1}=\pi / \mu\right\}$, since the $H$ flow is not complete on the submanifold $\left\{x_{1}<\pi / \mu\right\}$.

Alternatively, one can view the particles on the ring as distinguishable. This can be encoded mathematically by employing the phase space

$$
\Omega^{\prime} \equiv \widetilde{\Omega} / \mathbb{Z}^{\prime}
$$

where $\mathbb{Z}^{\prime}$ denotes the $\mathbb{Z}$-action generated by

$$
\Gamma^{N}:(x, p) \mapsto\left(x_{1}+\frac{2 \pi}{\mu}, \ldots, x_{N}+\frac{2 \pi}{\mu}, p\right) .
$$

One way to coordinatize $\Omega^{\prime}$ is to take

$$
\Omega^{\prime} \simeq\left\{(x, p) \in \mathbb{R}^{2 N} \mid x \in F_{N}^{\prime}\right\}
$$

where

$$
F_{N}^{\prime} \equiv\left\{x \in G \mid \sum_{j=1}^{N} x_{j} \in\left(-\frac{\pi N}{\mu}, \frac{\pi N}{\mu}\right]\right\}
$$


Indeed, for a given $(\tilde{x}, \tilde{p}) \in \widetilde{\Omega}$ one can take

$$
x_{j}=\tilde{x}_{j}+\frac{2 \pi m}{\mu}, p_{j}=\tilde{p}_{j}, \quad j=1, \ldots, N,
$$

where $m \in \mathbb{Z}$ is uniquely determined.

These three interpretations of the type III Hamiltonian illustrate that the canonical coordinates $x$ and $p$ in Hamilton's equations (2.1) may have several meanings, depending on what one intends to model physically. The same ambiguity occurs for the type IV systems, since the graph of $\mathcal{P}(x)$ on $(0,2 \omega)$ has the same features as that of $1 / \sin ^{2}(\pi x / 2 \omega)$. Thus, replacing $\mu$ by $\pi / \omega$ in the above, one obtains three mathematically and physically distinct versions of the elliptic system.

For all of the above Hamiltonians $H(x, p)$, the function

$$
P \equiv \sum_{j=1}^{N} p_{j}
$$

belongs to $\mathcal{I}_{H}$; cf. (2.7). This expresses conservation of total momentum under the $H$ flow. (Reciprocally, the conservation of $H$ under the $P$ flow

$$
e^{y P}(x, p)=(x+(y, \ldots, y), p)
$$

amounts to translational invariance of the potential energy.) Thus the Hamiltonian $H$ is integrable for $N=2$, but this clearly holds true for any Hamiltonian of the form

$$
H\left(x_{1}, x_{2}, p_{1}, p_{2}\right)=\frac{p_{1}^{2}}{2}+\frac{p_{2}^{2}}{2}+U\left(x_{1}-x_{2}\right) .
$$

The Calogero-Moser Hamiltonians, however, remain integrable for $N>$ 2. The existence of $N$ independent integrals can be most easily seen via a so-called Lax pair formulation of the Hamilton equations. This concept is also crucial in the context of infinite-dimensional integrable systems, such as the sine-Gordon field theory. We continue by describing it in a rather general form that can be applied to the latter context, too.

Specifically, suppose that $L(t), M(t), t \in \mathbb{R}$, are two families of (linear) operators on a (separable, complex) Hilbert space $\mathcal{H}$ such that

$$
\dot{L}=[M, L], \quad \forall t \in \mathbb{R} .
$$

(When $\operatorname{dim} \mathcal{H}=\infty$ and $L(t), M(t)$ are unbounded operators, one needs additional assumptions. Such technicalities will be ignored here.) Then the spectrum of $L(t)$ is constant in $t$. This assertion can be quickly proved by using linear ODE methods, as follows.

We begin by noting that the ODE $\dot{U}=M U$ has a unique solution $U(t)$ such that $U(0)=1$. Specifically, iteration of the corresponding integral 
equation

$$
U(t)=1+\int_{0}^{t} d s M(s) U(s)
$$

yields the solution

$$
U(t)=1+\sum_{n=1}^{\infty} \int_{0}^{t} d s_{1} \cdots \int_{0}^{s_{n-1}} d s_{n} M\left(s_{1}\right) \cdots M\left(s_{n}\right) .
$$

(Depending on subcultures, this formula is referred to variously as the Volterra expansion/variation of constants formula/Dyson expansion/timeordered exponential/product integral.) Defining now the operator family

$$
K(t) \equiv U(t) L(0) U(t)^{-1}
$$

we see that $K(t)$ is similar to $L(0)$, and so its spectrum equals that of $L(0)$. To complete the proof, it therefore remains to show that $K(t)$ equals $L(t)$. Since this is true for $t=0$, we need only prove $\dot{K}=[M, K]$. (Indeed, this linear ODE again has a solution that is uniquely determined by its initial value.) But this readily follows from (2.32) by using $0=\left(U U^{-1}\right)^{\circ}=$ $U\left(U^{-1}\right)^{\cdot}+M$.

For a finite-dimensional Hilbert space $\mathcal{H}=\mathbb{C}^{l}$ a second proof applies; this proof will also lead to the conserved Hamiltonians we are looking for. Let us define the power traces

$$
H_{k}=\frac{1}{k} \operatorname{Tr} L^{k}, \quad k=1,2, \ldots
$$

Using cyclicity of the trace, we then get

$$
\dot{H}_{k}=\operatorname{Tr}\left(\dot{L} L^{k-1}\right)=\operatorname{Tr}\left(M L^{k}-L M L^{k-1}\right)=0
$$

so that the traces are $t$-independent. Now write

$$
\operatorname{det}\left(1_{l}+\lambda L\right)=\sum_{k=0}^{\infty} \lambda^{k} S_{k}
$$

Thus, $S_{k}$ equals the sum of all $k$ th order principal minors of $L$ for $k \leq l$ and vanishes for $k>l$. These symmetric functions of $L$ are polynomials in the power traces $H_{k}$ (and vice versa), so we may conclude $\dot{S}_{k}=0$. Consequently, $L(t)$ has $t$-independent spectrum, as claimed.

We mention in passing that the polynomial relations between the $S_{k}$ and the $H_{k}$ boil down to the well-known Newton identities that connect the coefficients of a polynomial with the sums of its root powers. The relations can be explicitly determined from the formal power series identity

$$
1+\lambda S_{1}+\lambda^{2} S_{2}+\lambda^{3} S_{3}+\cdots=\exp \left(\lambda H_{1}-\lambda^{2} H_{2}+\lambda^{3} H_{3}-\cdots\right) .
$$


(To see that this holds for an arbitrary $l \times l$ matrix $L$, first take $L=$ $\operatorname{diag}\left(\lambda_{1}, \ldots, \lambda_{l}\right)$; then (2.34) is clear from the Taylor series $\ln (1+x)=$ $-\sum(-x)^{j},|x|<1$. But then (2.34) follows for diagonalizable $L$, since traces and determinants are invariant under similarity. Now diagonalizable $L$ are dense in $M_{l}(\mathbb{C})$, so the polynomial identities obtained by equating powers of $\lambda$ indeed hold true for arbitrary $l \times l$ matrices.)

To apply this isospectrality result to a Hamiltonian $H$ on a symplectic manifold $\langle\Omega, \omega\rangle$, it suffices to construct $M_{l}(\mathbb{C})$-valued functions $L$ and $M$ on $\Omega$ such that the pair $K(t) \equiv K(u(t)), K=L, M$, satisfies (2.31). (Of course, $u(t)$ denotes the $H$ flow; the abuse of notation occurring here is standard.) Indeed, whenever this can be done, the power traces of $L$ (or, equivalently, its symmetric functions) will generate a subalgebra of $\mathcal{I}_{H}$ (cf. the paragraph containing (2.7)). At this point it should be emphasized that there are no general methods for obtaining Lax pairs: They have to be pulled out of a hat. Moreover, when they exist, they are highly nonunique.

For the above Calogero-Moser Hamiltonians Lax pairs were found some twenty years ago. As it has turned out, a suitable choice for the Lax matrix $L$ not only yields $N$ integrals in involution, but also yields a key tool for constructing action-angle maps. We continue by detailing such a choice of $L$ and an associated $M$ for the hyperbolic case, and then exploit $L$ to derive various results of physical interest. Subsequently, the three remaining cases will be discussed briefly.

Our type II Lax pair is defined by taking $l=N$ and

$$
\begin{gathered}
L_{j k}=\delta_{j k} p_{j}+i g\left(1-\delta_{j k}\right) \frac{\mu}{2 \operatorname{sh} \mu\left(x_{j}-x_{k}\right) / 2}, \quad j, k=1, \ldots, N, \\
M_{j k}=\frac{i g \mu^{2}}{4}\left(-\delta_{j k} \sum_{l \neq j} \frac{1}{\operatorname{sh}^{2} \mu\left(x_{j}-x_{l}\right) / 2}\right. \\
\left.+\left(1-\delta_{j k}\right) \frac{\operatorname{ch} \mu\left(x_{j}-x_{k}\right) / 2}{\operatorname{sh}^{2} \mu\left(x_{j}-x_{k}\right) / 2}\right) .
\end{gathered}
$$

To verify that $(2.31)$ is obeyed for this choice, we first calculate

$$
\begin{aligned}
\dot{L}_{j j}=\dot{p}_{j} & =-\partial_{x_{j}} H \\
{[M, L]_{j j}=\sum_{k \neq j}\left(M_{j k} L_{k j}-L_{j k} M_{k j}\right) } & =\frac{g^{2} \mu^{2}}{4} \sum_{k \neq j} \frac{\operatorname{ch} \mu\left(x_{j}-x_{k}\right) / 2}{\operatorname{sh}^{3} \mu\left(x_{j}-x_{k}\right) / 2} .
\end{aligned}
$$

Recalling (2.13) and (2.19), we see that the two right-hand sides are indeed equal. The off-diagonal elements yield

$$
\dot{L}_{j k}=\frac{-i g \mu^{2}}{4} \frac{\operatorname{ch} \mu\left(x_{j}-x_{k}\right) / 2}{\operatorname{sh}^{2} \mu\left(x_{j}-x_{k}\right) / 2}\left(\dot{x}_{j}-\dot{x}_{k}\right)
$$

which should be compared to 


$$
\begin{aligned}
{[M, L]_{j k} } & \equiv M_{j k} L_{k k}-L_{j j} M_{j k}+R_{j k} \\
& =\frac{i g \mu^{2}}{4} \frac{\operatorname{ch} \mu\left(x_{j}-x_{k}\right) / 2}{\operatorname{sh}^{2} \mu\left(x_{j}-x_{k}\right) / 2}\left(p_{k}-p_{j}\right)+R_{j k} .
\end{aligned}
$$

From this one infers that it remains to show $R_{j k}=0$. To this end, one can combine the terms with summation index $l \neq j, k$ and use the elementary functional equation

$$
(\operatorname{ch} a \operatorname{sh} b-\operatorname{sh} a \operatorname{ch} b) \operatorname{sh}(a+b)+\operatorname{sh}^{2} a-\operatorname{sh}^{2} b=0
$$

with $a=\mu\left(x_{j}-x_{l}\right) / 2$ and $b=\mu\left(x_{l}-x_{k}\right) / 2$.

The upshot is, that the power traces $H_{k}$ and symmetric functions $S_{k}$ of the Lax matrix (2.35) are conserved under the $H$ flow. Obviously, we have

$$
H_{1}=\operatorname{Tr} L=P, \quad H_{2}=\frac{1}{2} \operatorname{Tr} L^{2}=H,
$$

and

$$
H_{k}=\frac{1}{k} \sum_{j=1}^{N} p_{j}^{k}+U_{k}(x, p), \quad k>2,
$$

where $U_{k}$ has degree $<k$ in $p_{1}, \ldots, p_{N}$.

From this one readily infers that the gradients of $H_{1}, \ldots, H_{N}$ are linearly independent on an open dense subset of $\Omega$. (Fixing $\lambda>0$, the determinant of the $N \times N$ matrix with rows $\lambda^{-k+1}\left(\nabla_{p} H_{k}\right)(x, \lambda p)$ is a real-analytic function on $\Omega$. Hence it either vanishes identically or on a closed nowhere dense subvariety of $\Omega$. Assuming the first case applies, one obtains a contradiction: taking $\lambda \rightarrow \infty$, the determinant converges to a Vandermonde determinant, which is nonzero for $p_{1}, \ldots, p_{N}$ distinct.) In fact, the gradients are independent on all of $\Omega$, as we will see later on.

Next, we turn to showing involutivity of the integrals. One way to prove this property proceeds via the long-time asymptotics of the flow. The first step of the argument (which is in essence due to Moser [33]) consists in showing that for any initial point $\left(x_{0}, p_{0}\right) \in \Omega$ one gets

$$
x_{j}(t) \sim x_{j}^{ \pm}+t p_{j}^{ \pm}, p_{j}(t) \sim p_{j}^{ \pm}, \quad t \rightarrow \pm \infty,
$$

with

$$
p_{N}^{-}>\cdots>p_{1}^{-}, \quad p_{N}^{+}<\cdots<p_{1}^{+} .
$$

In view of the repulsive character of the interparticle forces, this result is very plausible. The complete proof (which is quite subtle) exploits this physical intuition, and for brevity we skip it.

The second step is easy. Recalling first

$$
C_{k l} \equiv\left\{H_{k}, H_{l}\right\} \in \mathcal{I}_{H}
$$


(cf. the paragraph containing (2.7)), we obtain

$$
C_{k l}\left(x_{0}, p_{0}\right)=C_{k l}(x(t), p(t)), \quad \forall t \in \mathbb{R} .
$$

Now each term in $C_{k l}$ contains at least one factor $1 / \operatorname{sh}\left[\mu\left(x_{i}-x_{j}\right) / 2\right]$, so taking $t \rightarrow \infty$ in (2.45) and using (2.42), we deduce $C_{k l}=0$, as desired.

Combining the asymptotics (2.42) with the properties of the Lax matrix, we can easily arrive at further conclusions of physical interest. First, using (2.35) we deduce

$$
L(x(t), p(t)) \sim \operatorname{diag}\left(p_{1}^{ \pm}, \ldots, p_{N}^{ \pm}\right), \quad t \rightarrow \pm \infty .
$$

Second, combining (2.46) and isospectrality, we obtain

$$
p_{j}^{+}=p_{N-j+1}^{-}, \quad j=1, \ldots, N \text { (conservation of momenta). }
$$

Third, let us assume that the scattering map

$$
S:\left(x^{-}, p^{-}\right) \mapsto\left(x^{+}, p^{+}\right)
$$

is a canonical transformation. (This is plausible, since it amounts to a limit of $t$-dependent canonical transformations; cf. Section 5.2.) If we then define $\Delta_{j}$ by setting

$$
x_{N-j+1}^{+}=x_{j}^{-}+\Delta_{j}
$$

and use (2.47), it follows that $\partial \Delta_{j} / \partial x_{k}^{-}=0$. Thus, $\Delta_{j}$ depends only on $p^{-}$and can be determined by choosing $x_{1}^{-}, \ldots, x_{N}^{-}$such that the collisions take place approximately pairwise. But then one clearly gets

$$
\Delta_{j}\left(p^{-}\right)=\sum_{k \neq j} \delta\left(p_{j}^{-}, p_{k}^{-}\right) \text {(factorization) }
$$

where $\delta\left(p, p^{\prime}\right)$ denotes the position shift incurred in a 2-particle collision with asymptotic momenta $p, p^{\prime}$.

The two properties (2.47) and (2.48) are the hallmark of soliton scattering: the Calogero-Moser particles scatter just as the soliton solutions to various two-dimensional integrable PDEs (such as the Korteweg-de Vries and sine-Gordon equations). In Section 5 we shall elaborate on the above findings, and show in particular that the maps $(x, p) \mapsto\left(x^{ \pm}, p^{ \pm}\right)$may be viewed as action-angle maps. Here, we only add that completeness of the flow $\exp \left(t_{k} H_{k}\right)$ (and hence Liouville integrability of $H$ ) is easily established. Indeed, conservation of $H$ yields an upper bound on $\left|p\left(t_{k}\right)\right|$ and a nonzero lower bound on particle distances. Using these bounds one infers that $\left|d x_{j} / d t_{k}\right|$ is bounded above, so escape to infinity in finite time cannot occur either.

Next, we take a brief look at the remaining cases. First, the above discussion applies verbatim to the type I system: One need only send $\mu$ to 0 
in the equations (2.35), (2.36), and (2.38)-(2.39). Taking now $\mu \rightarrow i \mu$ in these equations, they clearly apply when $H$ is taken to be the Sutherland Hamiltonian (2.13), (2.20), and $\widetilde{\Omega}(2.24)$ is chosen as phase space. Changing (2.40) accordingly, it follows once more that one is dealing with an isospectral flow. As is easily checked, the trigonometric Lax matrix satisfies

$$
L(\Gamma(x, p))=S_{-}^{t} L(x, p) S_{-}, \quad(x, p) \in \widetilde{\Omega},
$$

where $\Gamma$ is the generator (2.25) and $S_{-}$the antiperiodic shift,

$$
S_{-} \equiv\left(\begin{array}{cccc}
0 & 1 & \ldots & 0 \\
\vdots & \vdots & \ddots & \vdots \\
0 & 0 & \ldots & 1 \\
-1 & 0 & \ldots & 0
\end{array}\right)
$$

From this similarity relation we deduce that the power traces on $\widetilde{\Omega}$ descend to smooth functions on the quotient manifolds $\Omega$ (2.26) and $\Omega^{\prime}(2.29)$. Independence and involutivity of $H_{1}, \ldots, H_{N}$ on all three phase spaces now follow by analytic continuation in $\mu$ from the hyperbolic case.

Of course, no scattering takes place in the Sutherland case. Choosing $\widetilde{\Omega}$ as phase space, the center of mass

$$
\frac{1}{N} \sum_{j=1}^{N} x_{j} \equiv X \in C^{\infty}(\widetilde{\Omega})
$$

moves uniformly along the line under the $H$ flow. The distances between the particles are bounded above by $2 \pi / \mu$, and one is therefore dealing with an oscillatory motion (classical $N$-particle "molecule").

The function $X$ is not invariant under $\Gamma$, so it does not descend to a smooth function on $\Omega$ and $\Omega^{\prime}$. (The physical picture of particles on a ring does not allow for an unambiguous center of mass position on the ring either.) The $X$ flow on $\widetilde{\Omega}$, given by

$$
e^{a X}(x, p)=\left(x, p-\left(\frac{a}{N}, \ldots, \frac{a}{N}\right)\right)
$$

is invariant under $\Gamma$, however. Therefore, it descends to a smooth symplectic flow on $\Omega$ and $\Omega^{\prime}$. Accordingly, the associated vector fields are locally, but not globally Hamiltonian. This reflects the fact that the quotient manifolds are not simply connected. More specifically, (2.26) and (2.29) entail $\pi_{1}(\Omega)=\pi_{1}\left(\Omega^{\prime}\right)=\mathbb{Z}$, since $\widetilde{\Omega}$ is convex and hence simply connected.

Replacing $2 \pi / \mu$ by $2 \omega$, the last two paragraphs also apply to the type IV system. In this case, too, a Lax pair formulation exists. Here, however, it turns out to be crucial to let $L$ and $M$ depend on an additional spectral parameter $\lambda \in \mathbb{C}$. Such a Lax pair was introduced by Krichever [34], who 
used the Lax matrix for constructing an action-angle map. His Lax matrix is expressed in terms of the Weierstrass $\sigma$-function $\sigma\left(x ; \omega, \omega^{\prime}\right)$, but both here and later on it is convenient to trade $\sigma(x)$ for the $2 \omega$-antiperiodic function $s(x)$ defined by

$$
s\left(x ; \omega, \omega^{\prime}\right) \equiv \sigma\left(x ; \omega, \omega^{\prime}\right) \exp \left(-\eta\left(\omega, \omega^{\prime}\right) \frac{x^{2}}{2 \omega}\right) .
$$

Moreover, we only detail the Lax matrix: We choose

$$
L_{j k}=\delta_{j k} p_{j}+i g\left(1-\delta_{j k}\right) \frac{s\left(x_{j}-x_{k}+\lambda\right)}{s(\lambda) s\left(x_{j}-x_{k}\right)}, \quad j, k=1, \ldots, N .
$$

This matrix is a similarity transform of Krichever's Lax matrix, so it yields the same symmetric functions and power traces. In particular, using the well-known identity

$$
\frac{\sigma(x+\lambda) \sigma(x-\lambda)}{\sigma^{2}(x) \sigma^{2}(\lambda)}=\mathcal{P}(\lambda)-\mathcal{P}(x)
$$

we obtain

$$
H_{2}=\frac{1}{2} \operatorname{Tr} L^{2}=H-\frac{1}{2} g^{2} N(N-1) \mathcal{P}(\lambda) .
$$

Moreover, in this case one readily verifies

$$
L(\Gamma(x, p))=S_{+}^{t} L(x, p) S_{+}, \quad(x, p) \in \widetilde{\Omega},
$$

where $\Gamma$ is given by (2.25) with $\mu \rightarrow \pi / \omega$ and $S_{+}$is the periodic shift,

$$
S_{+} \equiv\left(\begin{array}{cccc}
0 & 1 & \ldots & 0 \\
\vdots & \vdots & \ddots & \vdots \\
0 & 0 & \ldots & 1 \\
1 & 0 & \ldots & 0
\end{array}\right) .
$$

Therefore, the functions $H_{k} \in C^{\infty}(\widetilde{\Omega})$ descend to smooth functions on $\Omega$ and $\Omega^{\prime}$.

From our later account of the relativistic version of the type IV system it will transpire that the symmetric functions $S_{k}(\lambda)$ of $L(2.51)$ (and hence its power traces, too) are polynomials in $g, p_{j}$ and $\mathcal{P}\left(x_{j}-x_{k}\right), j, k=1$, $\ldots, N$, with $\lambda$-dependent coefficients that are real for $\lambda$ purely imaginary and not equal to $2 k \omega^{\prime}, k \in \mathbb{Z}$; furthermore, $S_{k}\left(\lambda_{1}\right)$ and $S_{l}\left(\lambda_{2}\right)$ commute also when $\lambda_{1} \neq \lambda_{2}$. At this point, this is very far from obvious, of course. But the linear independence of $d H_{1}, \ldots, d H_{N}$ on open dense subsets of the phase spaces $\widetilde{\Omega}, \Omega$ and $\Omega^{\prime}$ follows in the same way as before, and once we know $H$ is integrable, we can deduce its Liouville integrability (viz., completeness of the $H_{k}$ flows, $\left.k=1, \ldots, N\right)$ just as for the type III case. 
As we have seen above, the type I-III Hamiltonians $H$ may be viewed as specializations of the type IV Hamiltonian $H$; cf. (2.22). The relation of $L($ IV) $(2.51)$ to $L($ II $)(2.35)$ and its associated type III $(\mu \rightarrow i \mu)$ and type I $(\mu \rightarrow 0)$ versions remains to be explained, however. It is convenient to do so at the end of the next subsection.

\subsection{Toda Systems}

The Toda systems are defined by Hamiltonians of the form

$$
H=\frac{1}{2} \sum_{j=1}^{N} p_{j}^{2}+U(x)
$$

with $U(x)$ given by

$$
\begin{array}{ll}
\text { V. } a^{2}\left(\sum_{j=2}^{N} e^{\mu\left(x_{j}-x_{j-1}\right)}+e^{\mu\left(x_{1}-x_{N}\right)}\right), a, \mu>0 \quad \text { (periodic Toda) } \\
\text { VI. } \sum_{j=2}^{N} e^{\mu\left(x_{j}-x_{j-1}\right)}, \mu>0
\end{array}
$$

In both cases $U(x)$ is smooth on $\mathbb{R}^{N}$, so as phase space we should take

$$
\Omega \equiv G \times \mathbb{R}^{N}, \quad G \equiv \mathbb{R}^{N}
$$

Since $U(x)$ is positive, energy conservation yields an upper bound on $|p(t)|$, and so escape to infinity in finite time cannot occur. Hence, the $H$ flows of type $\mathrm{V}$ and VI are complete on $\Omega$. In the former case energy conservation also yields an upper bound on particle distances, so one gets an oscillatory motion for arbitrary initial states ("Toda molecule"). In the latter case the interparticle forces are repulsive, and so each initial state is a scattering state.

The existence of $N$ independent integrals can be established once more via a Lax pair formulation of the $H$ flow. Here, we only detail the Lax matrix $L$. For the periodic Toda system it is important to let $L$ depend on a spectral parameter $w \in \mathbb{C}^{*}$. Specifically, one can take

$$
\begin{aligned}
L(\mathrm{~V})_{j k}= & \delta_{j k} p_{j}+\delta_{j, k-1}+a^{2} \delta_{j, k+1} \exp \left(\mu\left(x_{j}-x_{j-1}\right)\right) \\
& -(i a)^{N} w \delta_{j N} \delta_{k 1}-a^{2}(i a)^{-N} w^{-1} \delta_{j 1} \delta_{k N} \exp \left(\mu\left(x_{1}-x_{N}\right)\right)
\end{aligned}
$$

so that

$$
H_{1}=\operatorname{Tr} L=P, \quad H_{2}=\frac{1}{2} \operatorname{Tr} L^{2}=H,
$$

(When $N=2$, one should add the constant $a^{2}(w+1 / w)$ to $H$.) For the nonperiodic case we choose

$$
L(\mathrm{VI})_{j k}=\delta_{j k} p_{j}+\delta_{j, k-1}+\delta_{j, k+1} \exp \left(\mu\left(x_{j}-x_{j-1}\right)\right)
$$


and then (2.58) holds true again.

Using the argument below (2.41), one deduces once more that the power traces $H_{1}, \ldots, H_{N}$ are independent. Taking for granted that $H_{3}, \ldots, H_{N} \in$ $\mathcal{I}_{H}$, one also infers completeness of the flows $\exp \left(t_{k} H_{k}\right)$ from $H$ being conserved. For the nonperiodic Toda case one can again exploit the repulsive character of the forces to prove (2.42) and (2.43), and then involutivity and the soliton scattering properties (2.47) and (2.48) follow in the same way as for the hyperbolic case.

Next, we substitute

$$
x_{j} \rightarrow x_{j}-2 j \mu^{-1} \ln a, \quad j=1, \ldots, N,
$$

in the Lax matrix $L(\mathrm{~V})(x, p)$, yielding a new matrix-valued function $L^{(a)}(x, p)$ on $\Omega$. Since (2.60) may be viewed as a canonical transformation on $\Omega$, the power traces of $L^{(a)}$ still Poisson commute. Now we clearly have

$$
\lim _{a \rightarrow 0} L^{(a)}=L(\mathrm{VI})
$$

so the latter Hamiltonians converge to the power traces of $L(\mathrm{VI})$. Therefore, the nonperiodic Toda system may be obtained as a limit of the periodic one.

To conclude this section, we detail similar limit relations between the Lax matrices for the six types of systems introduced above. These can be encoded in the following hierarchy:

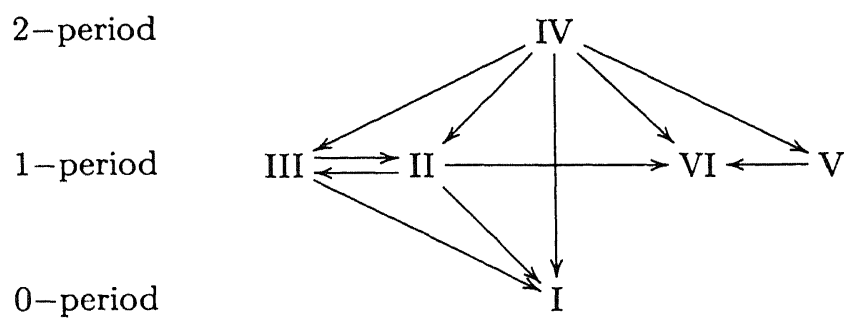

The transition V $\rightarrow$ VI was specified in the previous paragraph. Moreover, the relations between the type I-III Lax matrices were already described in the previous subsection: $L$ (II) and $L$ (III) (and hence $H$ and its commuting integrals) are related by analytic continuation in $\mu$, and taking $\mu \rightarrow 0$ yields $L(\mathrm{I})$. The relation between $H(\mathrm{IV})$ and $H(\mathrm{I})-H(\mathrm{III})$ can be read off from (2.22), but the transition $L(\mathrm{IV}) \rightarrow L(\mathrm{I})-L(\mathrm{III})$ is not obvious from (2.51) and (2.35) - if only because (2.51) depends on a spectral parameter and (2.35) does not. To elucidate this transition (and also for later purposes) we need more information on $s(x)$.

First, using the product representations for the Weierstrass $\sigma$-function, Legendre's relation, and $(2.50)$, we obtain

$$
s\left(x ; \omega, \omega^{\prime}\right)=\frac{2 \omega}{\pi} \sin \left(\frac{\pi x}{2 \omega}\right) \prod_{l=1}^{\infty} \frac{\left(1-q^{2 l} \exp (i \pi x / \omega)\right)(x \rightarrow-x)}{\left(1-q^{2 l}\right)^{2}},
$$




$$
\begin{aligned}
s\left(x ; \omega, \omega^{\prime}\right)=\frac{2 \omega^{\prime}}{i \pi} & \exp \left(-\frac{i \pi x^{2}}{4 \omega \omega^{\prime}}\right) \operatorname{sh}\left(\frac{i \pi x}{2 \omega^{\prime}}\right) \\
& \times \prod_{l=1}^{\infty} \frac{\left(1-\tilde{q}^{2 l} \exp \left(i \pi x / \omega^{\prime}\right)\right)(x \rightarrow-x)}{\left(1-\tilde{q}^{2 l}\right)^{2}}
\end{aligned}
$$

where

$$
q \equiv \exp \left(\frac{i \pi \omega^{\prime}}{\omega}\right), \quad \tilde{q} \equiv \exp \left(-\frac{i \pi \omega}{\omega^{\prime}}\right)
$$

Hence we have

$$
s\left(x ; \omega, \omega^{\prime}\right)= \begin{cases}x, & \omega=\infty, \omega^{\prime}=i \infty \\ \operatorname{sh}(\nu x) / \nu, & \omega=\infty, \omega^{\prime}=i \pi / 2 \nu, \\ \sin (\nu x) / \nu, & \omega=\pi / 2 \nu, \omega^{\prime}=i \infty .\end{cases}
$$

Thus, we obtain a hyperbolic Lax matrix depending on a spectral parameter $\lambda$ by substituting $\omega^{\prime}=i \pi / \mu$ in (2.51) and sending $\omega$ to $\infty$. If we now take $\operatorname{Re} \lambda \rightarrow \infty$ in this matrix, we obtain a similarity transform of (2.35). Substituting $\omega=\pi / \mu$ and letting $\omega^{\prime} \rightarrow i \infty$ yields a trigonometric Lax matrix depending on $\lambda$; taking $\operatorname{Im} \lambda \rightarrow \infty$ in the latter yields a similarity transform of the previous $L$ (III). Finally, putting $\omega^{\prime}=i \omega$ and letting $\omega \rightarrow \infty$ leads to a rational Lax matrix depending on $\lambda$; taking $\lambda \rightarrow \infty$, one gets the previous $L(\mathrm{I})$.

The transition IV $\rightarrow \mathrm{V}$ is quite nonobvious already at the level of the defining Hamiltonian $H$. To our knowledge, for $H$ this transition was first pointed out by Inozemtsev [35]. Here, we detail a more general limit $L(\mathrm{IV}) \rightarrow L(\mathrm{~V})$, which dates back to Ref. 5 . To begin with, $\omega^{\prime}$ should be replaced by $i \pi / \mu$, and a position shift

$$
x_{j} \rightarrow x_{j}-\frac{2 j \omega}{N}, \quad j=1, \ldots, N,
$$

should be made in (2.51). Then the power traces of the new Lax matrix (denoted by $L^{(s)}$ ) are smooth Poisson commuting functions on the shifted phase space

$$
\widetilde{\Omega}^{(s)} \equiv G^{(s)} \times \mathbb{R}^{N}
$$

where

$$
G^{(s)} \equiv\left\{\begin{array}{l|l}
x \in \mathbb{R}^{N} & \begin{array}{l}
x_{N}-2(N-1) \frac{\omega}{N}<\cdots<x_{2}-2 \frac{\omega}{N}<x_{1} \\
x_{1}-x_{N}<2 \frac{\omega}{N}
\end{array}
\end{array}\right\}
$$

Now we substitute

$$
\begin{aligned}
& g \rightarrow a \mu^{-1} \exp \left(\frac{\mu \omega}{N}\right), \\
& \lambda \rightarrow \omega-\frac{\delta}{\mu}, \quad \delta \equiv-i \pi+\ln w
\end{aligned}
$$


in $L^{(s)}$. Then it follows from a long, but straightforward calculation using (2.64) that the resulting matrix (still denoted $L^{(s)}$ ) satisfies

$$
\lim _{\omega \rightarrow \infty} L_{j k}^{(s)}=L(\mathrm{~V})_{j k}\left(i a \exp \left(\frac{\delta}{N}\right)\right)^{k-j} \exp \left(-\frac{\mu\left(x_{j}-x_{k}\right)}{2}\right) .
$$

(The infinite product in (2.64) may be omitted for $k-j<N / 2$, and contributes at most one factor otherwise.)

Note that the shifted configuration space $G^{(s)}(2.67)$ converges to the Toda configuration space $\mathbb{R}^{N}$ for $\omega \rightarrow \infty$. From a physical point of view, the new positions can be regarded as deviations from equilibrium positions. The distance $2 \omega / N$ between successive equilibrium positions is taken to $\infty$, and the coupling strength is simultaneously taken to $\infty$ in such a way that a finite interaction persists in the limit.

Next, we specify the transition II $\rightarrow$ VI. To this end we substitute

$$
\begin{aligned}
x_{j} & \rightarrow x_{j}+2 j \mu^{-1} \ln \epsilon, \quad j=1, \ldots, N, \\
g & \rightarrow \frac{1}{\mu \epsilon},
\end{aligned}
$$

in $L(\mathrm{II})(x, p)(2.35)$, yielding a new matrix $L^{(\epsilon)}(x, p)$ on a shifted phase space whose definition will be clear from the previous transition. Then one easily checks

$$
\lim _{\epsilon \rightarrow 0} L_{j k}^{(\epsilon)}=L(\mathrm{VI})_{j k}(-i)^{j-k} \exp \left(-\frac{\mu\left(x_{j}-x_{k}\right)}{2}\right) .
$$

(For the defining Hamiltonians this transition was already pointed out by Sutherland [36], as we recently learned. Independently, we obtained the more general transition (2.71) and its relativistic generalization in 1985.)

Finally, we describe how (a similarity transform of) $L(\mathrm{VI})$ can be reached directly from $L(I V)$ : Substituting

$$
\begin{aligned}
x_{j} & \rightarrow x_{j}-2 j\left(\frac{\omega}{N}-\frac{\ln \omega}{\mu}\right), \quad j=1, \ldots, N, \\
g & \rightarrow \frac{\exp (\mu \omega / N)}{\mu \omega}, \\
\lambda & \rightarrow \omega
\end{aligned}
$$

in $L(\mathrm{IV})$, the resulting matrix $\tilde{L}^{(s)}$ obeys

$$
\lim _{\omega \rightarrow \infty} \tilde{L}_{j k}^{(s)}=L(\mathrm{VI})_{j k}(-i)^{j-k} \exp \left(-\frac{\mu\left(x_{j}-x_{k}\right)}{2}\right) .
$$

(Once more, this can be verified by using the product representation (2.64) for $s(x)$.) 


\section{Relativistic Versions at the Classical Level}

\subsection{The Defining Dynamics and its Commuting Integrals}

In two space-time dimensions the nonrelativistic (Galilei) and relativistic (Poincaré) symmetry groups are semidirect products of boosts

$$
(t, x) \mapsto \begin{cases}(t, x+v t) & \text { (Galilei) } \\ \left(\left(t+\frac{v x}{c^{2}}\right)\left(1-\frac{v^{2}}{c^{2}}\right)^{-1 / 2},(x+v t)\left(1-\frac{v^{2}}{c^{2}}\right)^{-1 / 2}\right) & \text { (Lorentz) }\end{cases}
$$

(where $c$ denotes the speed of light) and space-time translations

$$
(t, x) \mapsto\left(t+a_{0}, x+a_{1}\right) .
$$

Clearly, the translations are 1-parameter diffeomorphism groups generated by vector fields

$$
X_{t} \equiv \partial_{t}, \quad X_{s} \equiv \partial_{x}
$$

whereas the Galilei boosts can be written

$$
\exp \left(v X_{b}\right)(t, x), \quad X_{b} \equiv t \partial_{x}
$$

To obtain a 1-parameter group of Lorentz boost diffeomorphisms, one should introduce the rapidity $\theta \in \mathbb{R}$ by setting

$$
\frac{v}{c}=\operatorname{th}\left(\frac{\theta}{c}\right)
$$

Then the Lorentz boost (3.1) reads

$$
(t, x) \mapsto\left(t \operatorname{ch}\left(\frac{\theta}{c}\right)+\frac{x}{c} \operatorname{sh}\left(\frac{\theta}{c}\right), x \operatorname{ch}\left(\frac{\theta}{c}\right)+c t \operatorname{sh}\left(\frac{\theta}{c}\right)\right)
$$

and so can be rewritten

$$
\exp \left(\theta X_{b}\right)(t, x), \quad X_{b} \equiv \frac{x}{c^{2}} \partial_{t}+t \partial_{x} .
$$

(Lorentz)

The vector fields $X_{t}, X_{s}$, and $X_{b}$ give rise to the space-time Lie algebras

$$
\left[X_{t}, X_{s}\right]=0, \quad\left[X_{t}, X_{b}\right]=X_{s}, \quad\left[X_{s}, X_{b}\right]= \begin{cases}0, & \text { (Galilei) } \\ X_{t} / c^{2} . & \text { (Poincaré) }\end{cases}
$$

Thus, the Galilei group and its Lie algebra may be viewed as deformations of the Poincaré group and its Lie algebra: The former result by taking $c \rightarrow \infty$ in the latter. 
Next, we observe that for any potential $V(x)$ the Hamiltonians

$$
\begin{aligned}
H & \equiv \frac{1}{2 m} \sum_{j=1}^{N} p_{j}^{2}+\sum_{1 \leq j<k \leq N} V\left(x_{j}-x_{k}\right), \\
P & \equiv \sum_{j=1}^{N} p_{j} \\
B & \equiv-m \sum_{j=1}^{N} x_{j}
\end{aligned}
$$

represent the Galilei Lie algebra. More precisely, one has

$$
\{H, P\}=0, \quad\{H, B\}=P, \quad\{P, B\}=N m,
$$

so that one obtains a central extension. But constant Hamiltonians generate trivial flows (the corresponding Hamiltonian vector field vanishes; cf. (2.6)), so we do obtain a faithful representation at the group level (assuming $H$, $P$, and $B$ generate complete flows).

Consider now the functions

$$
\begin{aligned}
& H \equiv m c^{2} \sum_{j=1}^{N} \operatorname{ch}\left(\frac{p_{j}}{m c}\right), \\
& P \equiv m c \sum_{j=1}^{N} \operatorname{sh}\left(\frac{p_{j}}{m c}\right), \\
& B \equiv-m \sum_{j=1}^{N} x_{j} .
\end{aligned}
$$

Clearly, these satisfy the Poincaré Lie algebra

$$
\{H, P\}=0, \quad\{H, B\}=P, \quad\{P, B\}=\frac{H}{c^{2}} .
$$

Physically, they describe a system of $N$ relativistic free (equal rest mass) particles in a slightly unorthodox way. Namely, instead of using the customary 1-particle momentum $k$ (in terms of which the kinetic energy reads $\left.\left(k^{2} c^{2}+m^{2} c^{4}\right)^{1 / 2}\right)$ and its canonically conjugate position $y$, we are using the rapidity variable $p / m$ and the variable $x$ canonically conjugate to $p$. The variables $p$ and $x$ then have the dimension of momentum and position, respectively.

Now recall this century's most widely known formula, $E=M c^{2}$. In words, this formula says that $M$ is determined not only by rest mass, but also by kinetic energy and any other form of energy due to interactions. 
Therefore, a quite simple way to take particle interactions into account for the above energy function is to replace it by

$$
H=\sum_{j=1}^{N} M_{j} c^{2}, \quad M_{j} \equiv m \operatorname{ch}\left(\frac{p_{j}}{m c}\right) V_{j}(x),
$$

with

$$
V_{j}(x) \equiv \prod_{k \neq j} f\left(x_{j}-x_{k}\right)
$$

Indeed, the function $V_{j}(x)$ (potential) then encodes the change in mass of particle $j$ due to its interaction with the remaining particles, just as the function $\operatorname{ch}\left(p_{j} / m c\right)$ encodes the change in mass due to its motion.

We should now ensure that the altered $H$ is still the time translation generator of (a phase space representation of) the Poincaré group. In contrast to the Galilei case (3.2)-(3.4), this is no longer true when both $P$ and $B$ are left unchanged. In fact, already translation invariance (viz., $\{H, P\}=0$ ) is violated when $P$ is left unchanged and $f(x)$ is not constant. The simplest choice is, therefore, to keep $B$ unchanged and change $P$ accordingly: It must read

$$
P=m c \sum_{j=1}^{N} \operatorname{sh}\left(\frac{p_{j}}{m c}\right) V_{j}(x)
$$

to yield the desired Poisson brackets $\{H, B\}=P$ and $\{P, B\}=H / c^{2}$.

But now we still have to satisfy the translation invariance constraint $\{H, P\}=0$. Assuming $f(x)$ is an even function, and calculating the Poisson bracket for (3.5) and (3.7), we see that it vanishes iff $f(x)$ satisfies the functional equation

$$
\sum_{j=1}^{N} \partial_{j} \prod_{k \neq j} f^{2}\left(x_{j}-x_{k}\right)=0
$$

Since $f(x)$ is assumed to be even, this yields no constraint for $N=2$. But for $N=3$ (3.8) can be rewritten

$$
\left|\begin{array}{ccc}
f^{2}(u) & f(u) f^{\prime}(u) & 1 \\
f^{2}(v) & f(v) f^{\prime}(v) & 1 \\
f^{2}(u+v) & -f(u+v) f^{\prime}(u+v) & 1
\end{array}\right|=0
$$

and this functional equation is known to be valid iff

$$
f^{2}(x)=a+b \mathcal{P}(x), \quad a, b \in \mathbb{C},
$$

where $\mathcal{P}$ is the Weierstrass function encountered in the previous sections. 
More generally, (3.8) turns out to be valid for $N>3$ when (3.9) holds [3]. (For $N>3$ it is not known, however, whether other solutions to (3.8) exist.) Therefore, we obtain relativistic analogs of the Hamiltonians of type I-IV when we take

$$
f(x)=\left(s^{2}\left(\frac{i g}{m c}\right)\left[\mathcal{P}\left(\frac{i g}{m c}\right)-\mathcal{P}(x)\right]\right)^{1 / 2}, \quad g>0 .
$$

(The radicand is positive, and we take positive square roots whenever this is the case.) In view of (2.22) and (2.65) this specializes to

$$
f(x)= \begin{cases}\left(1+g^{2} / m^{2} c^{2} x^{2}\right)^{1 / 2}, & (\text { I) } \\ \left(1+\sin ^{2}(\nu g / m c) / \operatorname{sh}^{2}(\nu x)\right)^{1 / 2}, & \text { (II), } \\ \left(1+\operatorname{sh}^{2}(\nu g / m c) / \sin ^{2}(\nu x)\right)^{1 / 2}, & \text { (III) } .\end{cases}
$$

We shall henceforth take $m=1$, just as we did in the nonrelativistic setting (2.13). For type I-III it is then routine to verify that one has

$$
\begin{gathered}
\lim _{c \rightarrow \infty}\left(H_{\mathrm{rel}}-N c^{2}\right)=H_{\mathrm{nr}}, \\
\lim _{c \rightarrow \infty} P_{\mathrm{rel}}=P_{\mathrm{nr} .}
\end{gathered}
$$

Here, $H_{\mathrm{nr}}$ denotes the nonrelativistic Hamiltonian (2.13) with $V(x)$ given by $(2.18)(2.20)$, and $H_{\text {rel }}$ denotes the relativistic Hamiltonian (3.5), with $V_{j}\left(x_{1}, \ldots, x_{N}\right)$ and $f(x)$ given by (3.6) and (3.11), respectively; similarly, $P_{\mathrm{nr}}$ and $P_{\text {rel }}$ denote (3.3) and (3.7), respectively. To obtain the corresponding limits for type IV, one needs

$$
\mathcal{P}(\epsilon)=\epsilon^{-2}+O\left(\epsilon^{2}\right), s(\epsilon)=\epsilon-\frac{\epsilon^{3} \eta}{2 \omega}+O\left(\epsilon^{5}\right), \quad \epsilon \rightarrow 0 .
$$

Then it is clear that (3.13) still holds, whereas (3.12) should be replaced by

$$
\begin{aligned}
\lim _{c \rightarrow \infty}\left(H_{\mathrm{rel}}-N c^{2}\right) & =\frac{1}{2} \sum_{j=1}^{N} p_{j}^{2}+g^{2} \sum_{\substack{j, k=1 \\
j<k}}^{N}\left(\mathcal{P}\left(x_{j}-x_{k}\right)+\frac{\eta}{\omega}\right) \\
& =H_{\mathrm{nr}}+\frac{g^{2} N(N-1) \eta}{2 \omega} .
\end{aligned}
$$

(To check that this is consistent with the type I-III limits (3.12), one should use

$$
\begin{aligned}
& \lim _{\omega \rightarrow \infty} \frac{\eta(\omega, i \pi / 2 \nu)}{\omega}=-\frac{\nu^{2}}{3} \\
& \lim _{\omega^{\prime} \rightarrow i \infty} \frac{\eta\left(\pi / 2 \nu, \omega^{\prime}\right)}{\omega}=\frac{\nu^{2}}{3}
\end{aligned}
$$


cf. also (2.22).)

Next, let us assume that the potential $V_{j}(x)$ in (3.5) and (3.7) has a "nearest neighbor" structure,

$$
V_{j}(x)=f_{T}\left(x_{j+1}-x_{j}\right) f_{T}\left(x_{j}-x_{j-1}\right)
$$

so as to mimic the interactions in the Toda systems. Then one easily verifies that one has $\{H, P\}=0$ iff

$$
\sum_{j=1}^{N} \partial_{j}\left(f_{T}^{2}\left(x_{j+1}-x_{j}\right) f_{T}^{2}\left(x_{j}-x_{j-1}\right)\right)=0 .
$$

Now it is not an easy matter to verify the functional equations (3.8), even for the rational case. In contrast, it is very simple to check that (3.14) is satisfied when one takes

$$
f_{T}^{2}(x)=a+b e^{\mu x}, \quad a, b \in \mathbb{C},
$$

with the convention

$$
\begin{array}{ll}
x_{0} \equiv x_{N}, & x_{N+1} \equiv x_{1} \\
x_{0} \equiv \infty, & x_{N+1} \equiv-\infty
\end{array}
$$

Indeed, the terms in (3.14) simply cancel in pairs. Choosing

$$
f_{T}(x) \equiv\left(1+a^{2} c^{-2} e^{\mu x}\right)^{1 / 2}, \quad \begin{cases}a>0 & (\mathrm{~V}) \\ a=1 & (\mathrm{VI})\end{cases}
$$

it is also easy to verify (3.12) and (3.13).

The mathematical upshot of the above physical reasoning is that insistence on the structure (3.5)-(3.7) for the Hamiltonians $H$ and $P$, together with the requirement $\{H, P\}=0$, has led us to a 1-parameter generalization of the commuting Hamiltonians $H$ and $P$ from Section 2. The former Hamiltonians can be rewritten

$$
H=\frac{S_{1}+S_{-1}}{2 \beta^{2}}, \quad P=\frac{S_{1}-S_{-1}}{2 \beta},
$$

where we have introduced a (more convenient) new parameter

$$
\beta \equiv \frac{1}{c}
$$

and the functions

$$
\begin{aligned}
S_{ \pm k} \equiv & \sum_{\substack{I \subset\{1, \ldots, N\} \\
|I|=k}} \exp \left( \pm \beta \sum_{i \in I} p_{i}\right) \\
& \cdot \begin{cases}\prod_{\substack{i \in I \\
j \notin I}} f\left(x_{i}-x_{j}\right) & (\mathrm{I}-\mathrm{IV}) \\
\prod_{\substack{i \in I \\
i+1 \notin I}} f_{T}\left(x_{i+1}-x_{i}\right) \prod_{\substack{i \in I \\
i-1 \notin I}} f_{T}\left(x_{i}-x_{i-1}\right) & (\mathrm{V}, \mathrm{VI})\end{cases}
\end{aligned}
$$


with $k=1, \ldots, N$. Since $H$ and $P$ commute, the "light cone Hamiltonians" $S_{1}$ and $S_{-1}$ commute as well.

Next, choosing $|I|=N$ in (3.19), we clearly get Hamiltonians

$$
S_{ \pm N}=\exp \left( \pm \beta\left(p_{1}+\cdots+p_{N}\right)\right)
$$

that commute with $S_{ \pm k}, k<N$, since only position differences occur in (3.19). It is therefore natural to conjecture that all of the functions $S_{k}$, $\pm k \in\{1, \ldots, N\}$, Poisson commute. Now with some perseverance, it can be verified that the commutators $\left\{S_{k}, S_{l}\right\}$ vanish for any $k, l \in\{ \pm 1, \ldots, \pm N\}$ and $N>1$ iff the functions $f$ and $f_{T}$ satisfy the functional equations

$$
\begin{aligned}
0= & \sum_{I \subset\{1, \ldots, N\}}\left(\sum_{i \in I} \partial_{i}\right) \\
& \cdot \begin{cases}\prod_{\substack{i \in I \\
j \notin I}} f^{2}\left(x_{i}-x_{j}\right) & (\mathrm{I}-\mathrm{IV}) \\
\prod_{\substack{i \in I \\
i+1 \notin I}} f_{T}^{2}\left(x_{i+1}-x_{i}\right) \prod_{\substack{i \in I \\
i \in 1 \notin I}} f_{T}^{2}\left(x_{i}-x_{i-1}\right) & (\mathrm{V}, \mathrm{VI})\end{cases}
\end{aligned}
$$

for any $k \in\{1, \ldots, N\}$ and $N>1$. For $k=1$ these equations clearly reduce to the functional equations (3.8) and (3.14) encoding relativistic invariance. But even for the Toda case, it is not easy to prove directly that these functional equations are satisfied for $k>1$, too. A direct proof that the equations (3.20) are valid in the Toda case can be found in Appendix A of [37]. As we shall see in Section 4.3, they are valid for the $\mathcal{P}$-function, too, but in that case no direct proof is known.

Let us now restrict attention to $S_{1}, \ldots, S_{N}$. (One easily verifies that

$$
S_{-k}=\frac{S_{N-k}}{S_{N}}, \quad k=1, \ldots, N .
$$

In particular, $H$ and $P$ may be viewed as functions of $S_{1}, \ldots, S_{N}$.) These functions yield smooth Poisson commuting Hamiltonians on the type-dependent phase spaces described in Section 2.2. (Notice that for type III and IV the functions (3.19) are manifestly invariant under the map (2.25) and its type IV analog.) In all cases the functions $V_{j}(x)$ are bounded away from 0 , just as the functions $\operatorname{ch}\left(\beta p_{j}\right)$. Therefore, conservation of $H$ yields an upper bound on $|p|$ and a nonzero lower bound on particle distances, and so completeness of the flows $\exp \left(t_{j} S_{j}\right), j=1, \ldots, N$, follows in the same way as in the nonrelativistic setting. However, the scaling argument below (2.41) no longer applies, so independence of $S_{1}, \ldots, S_{N}$ must be shown by other means.

A simple argument yielding independence for all cases at once now follows. View the determinant of the $N \times N$ matrix with rows $\nabla_{p} S_{k}$ as a polynomial in $e_{j}=\exp \left(\beta p_{j}\right), j=1, \ldots, N$, with $x$-dependent coefficients. Expanding the determinant, the product of the diagonal elements yields 
in particular a monomial $e_{1}^{N} e_{2}^{N-1} \cdots e_{N}$ with a positive coefficient. A moment's thought now shows that none of the remaining $N !-1$ products can yield such a monomial. Therefore, the determinant cannot vanish identically, and independence results.

From the preceding three paragraphs we deduce that the light cone Hamiltonian $S_{1}$ is Liouville integrable. (Of course, this is true for $H$, too, but it is more convenient to focus on $S_{1}$.) Since we know its commuting integrals explicitly, it might seem irrelevant to try and find a Lax pair formulation for the flow it generates. It turns out, however, that a special choice of Lax matrix $L$ makes it possible to derive some crucial results, as will become clear later on. (We do not need a Lax pair, however. Note in this connection that $M$ depends on the dynamics $S_{k}$ one selects, whereas $L$ encodes all dynamics of interest simultaneously.)

Before we detail Lax matrices, we would like to settle two issues for which a Lax matrix is not needed-in contrast to the nonrelativistic situation. First, specializing to the systems of type I, II, and VI, the repulsive character of the interparticle potentials $f\left(x_{j}-x_{k}\right)$ and $f_{T}\left(x_{j}-x_{k}\right)$, respectively, can be exploited to prove that the $S_{1}$ dynamics leads to soliton scattering. Specifically, Moser's argument can be adapted to the $S_{1}$ flow [3], yielding here

$$
x_{j}(t) \sim x_{j}^{ \pm}+\beta t \exp \left(\beta p_{j}^{ \pm}\right), p_{j}(t) \sim p_{j}^{ \pm}, \quad t \rightarrow \pm \infty,
$$

and, once more, (2.43). Therefore, the functions $s_{k}: t \mapsto S_{k}(x(t), p(t))$ reduce to symmetric functions of matrices $L^{ \pm} \equiv \operatorname{diag}\left(\exp \left(\beta p_{1}^{ \pm}\right), \ldots, \exp \left(\beta p_{N}^{ \pm}\right)\right)$ for $t \rightarrow \pm \infty$. Since $s_{k}(t)$ is $t$-independent, the roots of the polynomial $\lambda \mapsto\left|L^{-}-\lambda 1_{N}\right|$ equal those of $\lambda \mapsto\left|L^{+}-\lambda 1_{N}\right|$. Hence, conservation of momenta results; more in detail, (2.47) follows upon using (2.43). Then the factorization (2.48) of the asymptotic position shifts follows in the same way as before.

We point out in passing that (3.22) also entails that involutivity of $S_{1}$, $\ldots, S_{N}$ is a consequence of $S_{1}, \ldots, S_{N}$ being integrals of the $S_{1}$ flow. (Equivalently, for the systems of type I, II, and VI-and, by analytic continuation, for type III, too-the functional equations (3.20) follow once the special cases (3.8) and (3.14), respectively, are proved.) Indeed, the argument in the paragraph containing $(2.44)$ can easily be adapted to the systems involved.

The second issue we wish to address is the connection diagram (2.62), with regard to the functions $S_{1}, \ldots, S_{N}$. First of all, the connections between the type I-IV functions are obvious from (2.22) and (2.65); cf. (3.10) and (3.11). Second, we detail the transition V $\rightarrow$ VI: once again, one need only substitute (2.60) and take $a \rightarrow 0$ to obtain the nonperiodic Toda $S_{k}$ from the periodic ones.

Third, we consider the transition II $\rightarrow$ VI. To this end, we note first that the choice $\beta \mu g \in(0,2 \pi)$ is not the only one yielding a positive potential 


$$
f(x)=\left(1+\frac{\sin ^{2}(\beta \mu g / 2)}{\operatorname{sh}^{2}(\mu x / 2)}\right)^{1 / 2} .
$$

Indeed, we can allow $\beta \mu g=\pi-i d, d \in \mathbb{R}$, so as to let $\sin ^{2}(\beta \mu g / 2)$ vary over $(1, \infty)$, too. Taking now

$$
\beta \mu g \rightarrow \pi-2 i \ln \left(\frac{\beta}{\epsilon}\right)
$$

and substituting (2.69) in $f\left(x_{j}-x_{k}\right)$, we obtain a function $f_{j k}$ with limits

$$
f_{j k} \rightarrow \begin{cases}1, & |j-k|>1, \\ \left(1+\beta^{2} \exp \left[\mu\left(x_{j}-x_{j-1}\right)\right]\right)^{1 / 2}, & k=j-1 \\ \left(1+\beta^{2} \exp \left[\mu\left(x_{j+1}-x_{j}\right)\right]\right)^{1 / 2}, & k=j+1\end{cases}
$$

for $\epsilon \rightarrow 0$. Hence, these substitutions ensure that the hyperbolic $S_{k}$ converge to the nonperiodic Toda $S_{k}$ for $\epsilon \rightarrow 0$.

Fourth, we study the IV $\rightarrow \mathrm{V}$ limit. To this end we take $\omega^{\prime} \equiv i \pi / \mu$ and consider the elliptic potential, rewritten as

$$
f(x)=\left(\frac{s(x+i \beta g) s(x-i \beta g)}{s^{2}(x)}\right)^{1 / 2}
$$

cf. (3.10), (2.50), and (2.52). Using (2.64) this becomes

$$
f(x)=\rho(g) f_{\mathrm{II}}(x)\left(\prod_{l=1}^{\infty} \frac{\left(1-2 \tilde{q}^{2 l} \operatorname{ch} \mu(x+i \beta g)+\tilde{q}^{4 l}\right)(g \rightarrow-g)}{\left(1-2 \tilde{q}^{2 l} \operatorname{ch} \mu x+\tilde{q}^{4 l}\right)^{2}}\right)^{1 / 2}
$$

where $f_{\mathrm{II}}$ denotes the hyperbolic potential (3.23), and where

$$
\rho(g) \equiv \exp \left(\frac{\mu \beta^{2} g^{2}}{4 \omega}\right), \quad \tilde{q} \equiv \exp (-\mu \omega) .
$$

Now let us substitute (2.66) and

$$
i \beta g \rightarrow \frac{2 \omega}{N}+\frac{1}{\mu}(i \pi+2 \ln (a \beta))
$$

in $f\left(x_{j}-x_{k}\right)$, and study the three factors that arise from (3.27) for $\omega \rightarrow \infty$. First, the calculation in the previous paragraph (taking $\epsilon=\exp (-\mu \omega / N)$ ) shows that the $f_{\mathrm{II}}$ factor yields (3.25) with $\beta$ replaced by $a \beta$. Second, the infinite product converges to 1 unless $\{j, k\}=\{1, N\}$; in the latter case only the $l=1$ factor in the numerator does not converge to 1 , but rather to $1+a^{2} \beta^{2} \exp \mu\left(x_{1}-x_{N}\right)$.

Third, consider the constant prefactor $\rho(g)$ (3.28) with the substitution (3.29) in force. Clearly, the resulting factor is not real, and it goes to 0 for $\omega \rightarrow \infty$. However, this is easily remedied: We need only replace $f(x)$ by

$$
f_{r}(x) \equiv \frac{f(x)}{\rho(g)}
$$


to obtain renormalized functions $S_{r, k}$ with the desired type V limits when (2.66) and (3.29) are substituted. (These functions are, moreover, positive for $\beta \mu g=\pi-i d, d \in \mathbb{R}$.) This entails, in particular, that the involutivity of the periodic Toda $S_{k}$ may be viewed as a corollary of the involutivity of the elliptic $S_{k}$.

Finally, we specify the IV $\rightarrow$ VI transition. To this purpose we substitute (2.72) and

$$
i \beta g \rightarrow \frac{2 \omega}{N}+\frac{\kappa}{\mu}, \quad \kappa \equiv i \pi+2 \ln \left(\frac{\beta}{\omega}\right),
$$

in the above $S_{r, k}$ and take $\omega \rightarrow \infty$. This is readily verified to yield the type VI $S_{k}$; in this case the infinite product in (3.27) converges to 1 , and taking $\epsilon=\omega \exp (-\mu \omega / N)$ one can invoke (3.25) once again.

\subsection{Lax Matrices and Their Interrelationships}

Next, we turn to explicit Lax matrices, as promised above. Among other things, these will enable us to clarify the connection between the relativistic $S_{k}$ and their nonrelativistic counterparts.

We begin by specializing to the hyperbolic case Ii. Here, the key ingredient to arrive at the desired Lax matrix is an identity due to Cauchy, which is useful in various other contexts. It reads

$$
\operatorname{det}\left(\frac{1}{w_{j}-z_{k}}\right)_{j, k=1}^{N}=\prod_{j=1}^{N} \frac{1}{w_{j}-z_{j}} \prod_{\substack{j, k=1 \\ j<k}}^{N} \frac{\left(w_{j}-w_{k}\right)\left(z_{j}-z_{k}\right)}{\left(w_{j}-z_{k}\right)\left(z_{j}-w_{k}\right)}
$$

and can be proved, e.g., via induction on $N$. Substituting

$$
w_{j} \rightarrow \exp \mu\left(x_{j}+\frac{i \beta g}{2}\right), z_{k} \rightarrow \exp \mu\left(x_{k}-\frac{i \beta g}{2}\right), \quad \beta, \mu, g>0
$$

in the matrix occurring at the left-hand side of (3.32) yields a matrix $C$ with elements

$$
C_{j k}=\exp \left(-\mu \frac{x_{j}+x_{k}}{2}\right) \frac{\operatorname{sh}(i \beta \mu g / 2)}{\operatorname{sh} \mu\left(x_{j}-x_{k}+i \beta g\right) / 2}
$$

and determinant

$$
|C|=\exp \left(-\mu \sum_{j} x_{j}\right) \prod_{j<k} f^{-2}\left(x_{j}-x_{k}\right)
$$

where $f$ is given by (3.23).

If we now set

$$
L_{j k} \equiv e_{j} C_{j k} e_{k}
$$


where

$$
e_{j} \equiv \exp \left(\frac{\mu x_{j}+\beta p_{j}}{2}\right) \prod_{l \neq j} f\left(x_{j}-x_{l}\right)^{1 / 2},
$$

then it follows from (3.34) that the principal minor with indices $\left\{i_{1}, \ldots, i_{k}\right\}$ $\equiv I$ is given by

$$
\begin{aligned}
L(I) & =\left(\prod_{i \in I} e_{i}^{2}\right) C(I) \\
& =\left(\prod_{i \in I} \exp \left(\beta p_{i}\right)\right)\left(\prod_{i \in I} \prod_{\substack{l \neq i\\
}} f\left(x_{i}-x_{l}\right)\right) \prod_{\substack{j<k \\
j, k \in I}} f^{-2}\left(x_{j}-x_{k}\right) \\
& =\left(\prod_{i \in I} \exp \left(\beta p_{i}\right)\right) \prod_{\substack{i \in I \\
l \notin I}} f\left(x_{i}-x_{l}\right) .
\end{aligned}
$$

Therefore, the $k$ th symmetric function of $L$ reads

$$
S_{k}=\sum_{|I|=k} \exp \left(\beta \sum_{i \in I} p_{i}\right) \prod_{\substack{i \in I \\ j \notin I}} f\left(x_{i}-x_{j}\right) .
$$

Thus, it equals the above type II Hamiltonian $S_{k}(3.19)$, as anticipated by our notation.

Recalling now the nonrelativistic hyperbolic Lax matrix (2.35), we obtain, using obvious notation,

$$
L_{\mathrm{rel}}=1_{N}+\beta L_{\mathrm{nr}}+O\left(\beta^{2}\right), \quad \beta \rightarrow 0 .
$$

Taking $\beta$ to 0 in the determinant of the matrix $\beta^{-1}\left(L_{\text {rel }}-1_{N}\right)-\lambda 1_{N}$, we deduce

$$
S_{k, \mathrm{nr}}=\lim _{\beta \rightarrow 0} \beta^{-k} \sum_{l=0}^{k}(-)^{k+l}\left(\begin{array}{c}
N-l \\
N-k
\end{array}\right) S_{l, \text { rel }}
$$

Thus, the involutivity of the nonrelativistic type II functions follows from that of their relativistic counterparts. (Note that $L_{\text {rel }}$ is holomorphic at $\beta=0$, so that the $\beta \rightarrow 0$ limit may be interchanged with the partials in the Poisson brackets involved.)

Of course, we can now obtain type III and I Lax matrices from (3.35) by taking $\mu \rightarrow i \mu$ and $\mu \rightarrow 0$, respectively; then (3.38) and (3.39) hold true again. We can also derive a type VI Lax matrix from (3.35) by substituting (3.24) and (2.69) in the similarity transform

$$
\tilde{L}_{j k} \equiv \beta^{k-j} e_{j}^{2} C_{j k}
$$


and taking $\epsilon \rightarrow 0$. Indeed, using (3.25), this limit is easily calculated, yielding a matrix

$$
L(\mathrm{VI})_{j k}=\beta^{k-j} b_{j} E_{j k}
$$

where

$$
\begin{gathered}
b_{j} \equiv \exp \left(\beta p_{j}\right)\left(1+\beta^{2} \exp \left[\mu\left(x_{j+1}-x_{j}\right)\right]\right)^{1 / 2} \\
\times\left(1+\beta^{2} \exp \left[\mu\left(x_{j}-x_{j-1}\right)\right]\right)^{1 / 2}, \\
E_{j k} \equiv \begin{cases}1, & k-j=N-1, \ldots, 1,0, \\
a_{j}, & k-j=-1, \\
0, & k-j=-2, \ldots,-N+1,\end{cases} \\
a_{j} \equiv \beta^{2} \exp \left[\mu\left(x_{j}-x_{j-1}\right)\right]\left(1+\beta^{2} \exp \left[\mu\left(x_{j}-x_{j-1}\right)\right]\right)^{-1},
\end{gathered}
$$

(with the convention (3.16) in effect). It follows from the above that the symmetric functions of $L(\mathrm{VI})$ are indeed the type VI functions already defined. (It is not hard to verify this directly.) Comparing (3.41)-(3.44) and (2.59), it is also clear that (3.38) holds true once more, and so (3.39) follows for the nonperiodic Toda case, too.

We proceed by introducing a type IV Lax matrix: We choose

$$
L_{j k} \equiv d_{j} D_{j k}
$$

where

$$
\begin{gathered}
d_{j} \equiv \exp \left(\beta p_{j}\right) \prod_{l \neq j} f\left(x_{j}-x_{l}\right), f(x) \equiv\left(\frac{s(x+i \beta g) s(x-i \beta g)}{s^{2}(x)}\right)^{1 / 2} \\
D_{j k} \equiv \frac{s\left(x_{j}-x_{k}+\lambda\right)}{s(\lambda)} \frac{s(i \beta g)}{s\left(x_{j}-x_{k}+i \beta g\right)}
\end{gathered}
$$

It is immediate that this matrix is again related to its nonrelativistic analog (2.51) via (3.38), and that its trace equals the above elliptic Hamiltonian $S_{1}$. It is not at all immediate, but true, that all of its symmetric functions $\Sigma_{k}$ are proportional to the above elliptic $S_{k}$ (3.19). Specifically, we have

$$
\Sigma_{k}=s(\lambda)^{-k} s(\lambda-i \beta g)^{k-1} s(\lambda+(k-1) i \beta g) S_{k}, \quad k=1, \ldots, N .
$$

This assertion follows from a calculation similar to (3.37) and an identity generalizing Cauchy's identity (3.32), viz.,

$$
\begin{array}{rl}
\operatorname{det}\left(\frac{s\left(q_{j}-r_{k}+\lambda\right)}{s\left(q_{j}-r_{k}+\gamma\right)}\right)_{j, k=1}^{N} & s(\lambda-\gamma)^{N-1} s\left(\lambda+(N-1) \gamma+\sum_{j}\left(q_{j}-r_{j}\right)\right) \\
& \cdot \prod_{i<j} s\left(q_{i}-q_{j}\right) s\left(r_{j}-r_{i}\right) \prod_{i, j} s\left(q_{i}-r_{j}+\gamma\right)^{-1}
\end{array}
$$


This identity follows from (2.50) and Theorem B2 in Ref. 4. (Substantially the same identity was already proved (in a different way) by Frobenius [38]. We learned this fact from a paper by Raina [39], who supplies yet a third proof.)

Since (3.38) holds true with $L_{\text {rel }}$ given by (3.45) and $L_{\text {nr }}$ given by (2.51), we may conclude that (3.39) holds true, with $S_{l, \text { rel }}$ equal to $\Sigma_{l}(3.48)$. Now the $x$-dependence of the $\Sigma_{l}$ occurs solely in factors $\mathcal{P}\left(x_{j}-x_{k}\right)$ (recall (3.10)), and the $\lambda$-dependent prefactor in (3.48) is real for $\lambda$ purely imaginary and not equal to $2 l \omega^{\prime}, l \in \mathbb{Z}$. (This is because $s(x)$ is purely imaginary for $x$ purely imaginary; cf. (2.63).) Therefore, the assertions concerning the nonrelativistic $S_{k}$ made below (2.53) readily follow from (3.39).

Specializing the Lax matrix (3.45) to the rational, hyperbolic and trigonometric contexts, we obtain Lax matrices of type I, II, and III depending on a spectral parameter $\lambda \in \mathbb{C}$. Clearly, the previous Lax matrices (cf. (3.35)) result by taking $\lambda \rightarrow \infty, \operatorname{Re} \lambda \rightarrow \infty$ and $\operatorname{Im} \lambda \rightarrow \infty$, respectively (up to diagonal similarity factors).

Next, we use the above elliptic Lax matrix to arrive at a periodic Toda Lax matrix. Recall we already analyzed the IV $\rightarrow \mathrm{V}$ transition for the functions $S_{k}$; cf. (3.26)-(3.30). From these findings we infer that we should start from a renormalized Lax matrix

$$
L_{r} \equiv \rho(g)^{-N+1} L
$$

with the substitutions $\omega^{\prime}=i \pi / \mu,(2.66)$ and (3.29). The symmetric functions of $L_{r}$ are then given by

$$
\Sigma_{r, k}=\frac{s(\lambda-i \beta g)^{k-1} s(\lambda+(k-1) i \beta g)}{\rho(g)^{k(k-1)} s(\lambda)^{k}} S_{r, k}, \quad k=1, \ldots, N,
$$

with $S_{r, k}$ defined below (3.30). Now the latter substitutions ensure that $S_{r, k}$ has the desired type V limit $S_{k}(3.19)$ for $\omega \rightarrow \infty$. To get a finite limit for the prefactor in (3.51), we also substitute

$$
\lambda \rightarrow \frac{2 \omega}{N}+\frac{2}{\mu} \ln (a \beta)-\frac{N}{\mu} \ln (i a \beta)-\frac{1}{\mu} \ln w .
$$

Using (2.64) with $\omega^{\prime}=i \pi / \mu$, we see that then the limit $\omega \rightarrow \infty$ of $(3.51)$ is given by

$$
\Sigma_{k}= \begin{cases}\left(1+(i a \beta)^{N} w\right)^{k-1} S_{k}, & k=1, \ldots, N-1, \\ \left(1+(i a \beta)^{N} w\right)^{N-1}\left(1+(i a \beta)^{N} w^{-1}\right) S_{N}, & k=N .\end{cases}
$$

(The infinite products do not contribute, save for the $l=1$ term coming from the factor $s(\lambda+(k-1) i \beta g)$ when $k=N$.)

The above substitutions also ensure that $L_{r}$ (3.50) has a finite limit for $\omega \rightarrow \infty$. Specifically, the limit can be written

$$
\lim _{\omega \rightarrow \infty} L_{r, j k}=L(\mathrm{~V})_{j k}\left(i a \exp \left[\frac{i \pi+\ln w}{N}\right]\right)^{k-j} .
$$


The relativistic periodic Toda Lax matrix thus obtained reads explicitly

$$
L(\mathrm{~V})_{j k}=\beta^{k-j} b_{j} E_{j k}
$$

Here, one has

$$
\begin{gathered}
b_{j}=\exp \left(\beta p_{j}\right)\left(1+a^{2} \beta^{2} \exp \left[\mu\left(x_{j+1}-x_{j}\right)\right]\right)^{1 / 2} \\
\times\left(1+a^{2} \beta^{2} \exp \left[\mu\left(x_{j}-x_{j-1}\right)\right]\right)^{1 / 2} \\
E_{1 N}=\frac{1-(i a \beta)^{-N} w^{-1} a^{2} \beta^{2} \exp \left[\mu\left(x_{1}-x_{N}\right)\right]}{1+a^{2} \beta^{2} \exp \left[\mu\left(x_{1}-x_{N}\right)\right]} \\
E_{j k}=1, \quad k-j=N-2, \ldots, 1,0 \\
E_{j, j-1}=\frac{-(i a \beta)^{N} w+a^{2} \beta^{2} \exp \left[\mu\left(x_{j}-x_{j-1}\right)\right]}{1+a^{2} \beta^{2} \exp \left[\mu\left(x_{j}-x_{j-1}\right)\right]} \\
E_{j k}=-(i a \beta)^{N} w, \quad k-j=-2, \ldots,-N+1
\end{gathered}
$$

with the convention (3.15) in force. Its symmetric functions are given by (3.52) - a fact that is quite hard to see directly from (3.53)-(3.58).

It is immediate from the latter formulas that (3.38) holds true; cf. (2.57). From (3.39) it then follows that the nonrelativistic periodic Toda functions $S_{k}$ are $w$-independent for $k<N$, whereas for $k=N$ the $w$-dependence is given by an additive term $(i a)^{N}\left(w^{-1}+(-)^{N} w\right)$. It is also plain that when we substitute $(2.60)$ in $(3.53)-(3.58)$, yielding a new matrix $L^{(a)}$, then (2.61) holds true once more; cf. (3.41)-(3.44).

To complete our account of the connection diagram (2.62) at the level of relativistic Lax matrices, it remains to specify the direct transition IV $\rightarrow$ VI. To this end we take $\omega^{\prime}$ equal to $i \pi / \mu$ and substitute (2.72), (3.31), and

$$
\lambda \rightarrow \frac{2 \omega}{N}
$$

in $L_{r}$. Then the resulting matrix $\tilde{L}_{r}$ fulfils

$$
\lim _{\omega \rightarrow \infty} \tilde{L}_{r, j k}\left(\exp \left(\frac{\kappa}{N}\right)\right)^{j-k}=L(\mathrm{VI})_{j k} \beta^{j-k}
$$

where $\kappa$ is defined by (3.31).

We conclude this subsection with some remarks. First, we would like to point out that the function $s$ may be replaced by the function $\tilde{s}$ obtained from the right-hand side of (2.64) by omitting the exponential factor. Of course, this makes no difference for the type I-III systems, since $\tilde{\mathcal{s}}\left(x ; \omega, \omega^{\prime}\right)$ obeys $(2.65)$, too. Moreover, for the nonrelativistic elliptic Lax matrix (2.51) the substitution $s \rightarrow \tilde{s}$ amounts to a similarity transformation, so that the symmetric functions do not change. But in (3.45) this substitution leads to a similarity transform of $L_{r}$, and not of $L$; cf. (3.46), 
(3.47) and (3.50). Therefore, no renormalization is needed to obtain Toda limits; moreover, several similarity factors become simpler. Nevertheless, we have opted for using $s$, since this is the simplest choice at the quantum level. (Note that $\tilde{s}\left(x ; \omega, \omega^{\prime}\right)$ is not $2 \omega$-antiperiodic, in contrast to $s\left(x ; \omega, \omega^{\prime}\right)$.)

Second, we recall that our account of limit transitions has dealt with the connections encoded in the diagram (2.62) both for the nonrelativistic and for the relativistic systems. Viewing the two diagrams as stacked on top of each other, we have also detailed the vertical limits $\mathcal{S}_{\text {rel }} \rightarrow \mathcal{S}_{\text {nr }}, \mathcal{S}=$ IVI. We have, however, not analyzed skew limits, thus far. One of these is of particular interest (especially at the quantum level), viz., the limit $\mathrm{II}_{\text {rel }} \rightarrow \mathrm{VI}_{\mathrm{nr}}$. In contrast to the detours via the $\mathrm{II}_{\mathrm{nr}}$ or $\mathrm{VI}_{\text {rel }}$ systems, one should keep $\beta g$ fixed; specifically, setting

$$
\begin{gathered}
\frac{\beta \mu g}{2} \equiv \tau \in(0, \pi), \\
x_{j} \rightarrow x_{j}+2 j \mu^{-1} \ln \left(\frac{\beta}{2 \sin \tau}\right),
\end{gathered}
$$

in $\tilde{L}(3.40)$, one obtains a matrix $L^{(\tau)}$ for which one easily verifies

$$
L^{(\tau)}=1_{N}+\beta L^{(\tau)}\left(\mathrm{VI}_{\mathrm{nr}}\right)+O\left(\beta^{2}\right), \quad \beta \rightarrow 0,
$$

where

$$
L^{(\tau)}\left(\mathrm{VI}_{\mathrm{nr}}\right)_{j k} \equiv L\left(\mathrm{VI}_{\mathrm{nr}}\right)_{j k}\left(1-e^{-2 i \tau}\right)^{k-j}
$$

Consequently, the type II relativistic $S_{k}$ (with (3.59) and (3.60) in force) give rise to the type VI nonrelativistic $S_{k}$ as detailed by (3.39).

Third, we would like to point out that there exist additional systems (denoted $\mathrm{III}_{\mathrm{b}}$ and $\mathrm{IV}_{\mathrm{b}}$ ) that are related to the above $\mathrm{III}_{\text {rel }}$ and $\mathrm{IV}_{\text {rel }}$ systems via the substitution $\beta \rightarrow i \beta$. To obtain real-valued Hamiltonians, one should consider, e.g.,

$$
I_{k} \equiv \frac{S_{k}+S_{-k}}{2}, \quad k=1, \ldots, N
$$

Moreover, taking $\omega \equiv \pi / \mu$ so as to handle both cases at once, one should work with a configuration space

$$
G \equiv\left\{\begin{array}{l|l}
x \in \mathbb{R}^{N} & \begin{array}{l}
x_{j}-x_{j+1}>\beta g, j=1, \ldots, N-1, \\
x_{1}-x_{N}<2 \pi / \mu-\beta g
\end{array}
\end{array}\right\} . \quad\left(\mathrm{III}_{\mathrm{b}}, \mathrm{IV}_{\mathrm{b}}\right)
$$

This is necessary to keep the potentials $f\left(x_{j}-x_{k}\right)$ positive, since one now has

$$
f(x)=\left(s^{2}(\beta g)[\mathcal{P}(\beta g)-\mathcal{P}(x)]\right)^{1 / 2}, \quad \beta, g>0 .
$$


Consequently, one needs to require

$$
N<\frac{2 \pi}{\beta \mu g}
$$

for $G$ to be nonempty.

The Hamiltonians $I_{1}, \ldots, I_{N}$ on the phase space $\widetilde{\Omega} \equiv G \times \mathbb{R}^{N}$ are not only invariant under the $\mathbb{Z}$-action generated by $\Gamma(2.25)$, but also under the $\mathbb{Z}^{N}$-action $p \mapsto p+2 \pi k / \beta, k \in \mathbb{Z}^{N}$. Quotienting out the resulting action of $\mathbb{Z} \ltimes \mathbb{Z}^{N}$ on $\widetilde{\Omega}$ yields a second phase space $\Omega$; alternatively, one can consider quotients by proper subgroups, yielding phase spaces interpolating between the maximal and minimal phase spaces $\widetilde{\Omega}$ and $\Omega$, respectively. The flows generated by the commuting Hamiltonians $I_{1}, \ldots, I_{N-1}$ and their quotients are not complete, but this can be remedied. Specifically, a suitable interpolating phase space $\Omega^{c}$ can be densely embedded in a phase space of the form $\mathbb{R}^{2} \times \mathbb{P}^{N-1}$, on which all flows are complete. Here, $\mathbb{P}^{k}$ denotes complex projective space, viewed as a compact symplectic manifold by equipping it with the symplectic form derived from the Fubini-Study metric. Using (2.63), it is readily verified that this completion for the $\mathrm{III}_{\mathrm{b}}$ case (which is detailed in Ref. 40) is also appropriate for the $\mathrm{IV}_{\mathrm{b}}$ system. (Of course, inclusion of these systems in the diagram (2.62) would lead to a further proliferation of arrows.)

Last but not least, one can obtain new systems of considerable physical interest via analytic continuation of the positions. A detailed study of these soliton-antisoliton systems can be found in Ref. 30 .

\section{Quantum Calogero-Moser and Toda Systems}

\subsection{Background: Quantum Mechanics/Hilbert Space Theory}

We choose as our starting point a classical phase space $\Omega$ of the cotangent bundle type (2.2), and a smooth real-valued Hamiltonian $H(x, p)$ on $\Omega \simeq G \times \mathbb{R}^{N}$. Then the state space of the associated quantum system consists of the unit vectors in the Hilbert space $L^{2}(G, d x)$ of square-integrable complex-valued functions; when $\psi$ is a unit vector, the integral of $|\psi(x)|^{2}$ over a subset $B$ of $G$ is interpreted as the probability to find the system position vector in the set $B$.

The quantization of the dynamics $H(x, p)$ is obtained by means of the canonical quantization substitution

$$
p_{j} \rightarrow-i \hbar \frac{\partial}{\partial x_{j}} \equiv \hat{p}_{j}, \quad j=1, \ldots, N,
$$

where $\hbar>0$ denotes Planck's constant. Since the quantum operators $\hat{p}_{j}$ and $x_{j}$ do not commute, this substitution may lead to ordering ambiguities. 
For the Calogero-Moser and Toda Hamiltonians (2.13), (2.18)-(2.21) and (2.54)-(2.56) this is not the case, however: (4.1) yields an unambiguous (linear) partial differential operator (PDO) of the form

$$
H=\frac{1}{2} \sum_{j=1}^{N} \hat{p}_{j}^{2}+U(x)
$$

with $U(x)$ real-analytic on the classical configuration space $G$.

We shall say that a PDO of the form (4.2) is integrable when there exist independent PDOs $I_{1}=H, I_{2}(x, \hat{p}), \ldots, I_{N}(x, \hat{p})$ that commute pairwise. Here, independent means by definition that there are no polynomial relations between $I_{1}, \ldots, I_{N}$. Moreover, the notation $I_{k}(x, \hat{p})$ is meant to indicate that a specific ordering in the so-called symbol $I_{k}(x, p)$ has been chosen whenever ordering ambiguities are present. It should be observed that this definition does not involve any Hilbert space notion: Commutativity of PDOs is an algebraic issue. (Of course, one does need some smoothness conditions on the coefficients.) Presently, we shall introduce a stronger notion of Hilbert integrability, which is tied to Hilbert space theory.

Next, consider the Hamiltonians (3.18)-(3.19), taking $f(x)=f_{T}(x)=$ 1 at first (free particles). Then the substitution (4.1) yields exponential dependence on $\hat{p}_{j}$. The obvious way to interpret this is in the sense of analytic difference operators (A $\Delta \mathrm{Os}$ ). Thus, for instance,

$$
\left(\exp \left(a \hat{p}_{j}\right) \psi\right)\left(x_{1}, \ldots, x_{N}\right) \equiv \psi\left(x_{1}, \ldots, x_{j}-i \hbar a, \ldots, x_{N}\right), \quad a \in \mathbb{R}
$$

where one insists that $\psi$ admit analytic continuation off the real axis--just as PDOs should act on differentiable functions.

Consider now the interacting case $f, f_{T} \neq 1$. Then one still has $S_{ \pm N}=$ $\exp \left( \pm \beta\left(p_{1}+\cdots+p_{N}\right)\right)$, so the quantum versions of $S_{ \pm N}$ are the same A $\triangle$ Os as before. But for $|k|<N$ an interpretation of $S_{k}=\sum_{|I|=|k|} T_{I}$ as an $\mathrm{A} \Delta \mathrm{O}$ is ambiguous: the $\mathrm{A} \Delta \mathrm{O} T_{I}(x, \hat{p})$ depends on the ordering of $x$ - and $p$ dependent factors specified for its symbol $T_{I}(x, p)$. In particular, the $\mathrm{A} \Delta \mathrm{O}$ $H(x, \hat{p})$ associated with the Hamiltonian $H$ that defines the relativistic version of the model at hand is not uniquely determined-by contrast to the PDO (4.2).

In view of this state of affairs we shall say that the Hamiltonians $S_{k}$, $k= \pm 1, \ldots, \pm N$, defined by (3.19) admit an integrable quantization when there exist pairwise commuting A $\Delta \mathrm{Os} S_{k}(x, \hat{p})$ with symbols $S_{k}(x, p)$ equal to $S_{k}$ (as functions on the classical phase space). Whenever this is the case, we shall use (3.18) to include quantum versions of $H$ and $P$ in the commutative A $\Delta \mathrm{O}$ algebra generated by $S_{ \pm 1}(x, \hat{p}), \ldots, S_{ \pm N}(\hat{p})$. As we will see in Section 4.3, all of the type I-VI systems do admit integrable quantizations. (Anticipating the outcome, we mention that one should first choose an ordering different from the one in (3.19) before substituting (4.1).) 
Once more, these definitions do not involve Hilbert space: With appropriate analyticity assumptions on the $x$-dependent coefficients understood, commutativity of $\mathrm{A} \Delta \mathrm{Os}$ is an algebraic issue. In Sections 4.2 and 4.3 we deal with (nonrelativistic) PDOs and (relativistic) A $\Delta O$ s in a purely algebraic way, and the remainder of this subsection has no relevance for the issues addressed there.

The following is, however, crucial for Section 6, where we aim to understand the PDOs and A $\Delta O$ s as well-defined Hilbert space operators. Let us begin by recalling some standard fare. For a given quantum Hamiltonian $H$ on the Hilbert space $L^{2}\left(\mathbb{R}^{N}, d x\right)$ (say) the time evolution is encoded in the time-dependent Schrödinger equation

$$
i \hbar \dot{\Psi}=H \Psi .
$$

To solve this, one first reduces it to the time-independent Schrödinger equation

$$
H \psi=E \psi
$$

by setting

$$
\Psi(t, x)=\exp \left(-\frac{i t E}{\hbar}\right) \psi(x) .
$$

Solutions of (4.5) for which $\psi$ is square-integrable correspond to bound states; when no such solutions exist, one should try and find bounded solutions and construct square-integrable solutions to (4.4) by building wave packets.

For a Hamiltonian of the form (4.2), the simplest example of the latter situation is the free case $U=0$. Taking first $N=1$ and $E>0$, there exist two linearly independent solutions to (4.5), viz., the plane waves $\exp ( \pm i p x / \hbar)$, with $p^{2} / 2=E$. These are bounded, in contrast to the two solutions of this form for $E<0$ : then $p$ is purely imaginary and the solution increases exponentially for $x \rightarrow \infty$ or $x \rightarrow-\infty$.

The Hilbert space $L^{2}(\mathbb{R}, d x)$ can be spanned by the former solutions, in the sense that wave packet superpositions

$$
\psi(x) \equiv \int_{-\infty}^{\infty} d p \exp \left(\frac{i p x}{\hbar}\right) \phi(p), \quad \phi \in C_{0}^{\infty}(\mathbb{R}),
$$

are dense. (At the quantum level we are dealing with complex-valued functions. Accordingly, $C^{\infty}$ stands for smooth and complex-valued in Sections 4 and 6-in contrast with our convention at the classical level. Recall the subscript 0 means compact support.) Then one has

$$
(H \psi)(x)=\int_{-\infty}^{\infty} d p \exp \left(\frac{i p x}{\hbar}\right) \frac{p^{2}}{2} \phi(p)
$$


and the time-dependent equation (4.4) is solved by

$$
\left(\exp \left(-\frac{i t H}{\hbar}\right) \psi\right)(x)=\int_{-\infty}^{\infty} d p \exp \left(\frac{i p x}{\hbar}-\frac{i E t}{\hbar}\right) \phi(p), \quad E \equiv \frac{p^{2}}{2}
$$

Moreover, a suitable normalization ensures that one obtains a unitary operator from the spectral representation space $L^{2}(\mathbb{R}, d p)$ onto $L^{2}(\mathbb{R}, d x)$. Specifically, one should choose

$$
(\mathcal{E} \phi)(x) \equiv(2 \pi \hbar)^{-1 / 2} \int_{-\infty}^{\infty} d p \exp \left(\frac{i p x}{\hbar}\right) \phi(p)
$$

so as to obtain a unitary $\mathcal{E}$. This operator is a simple example of an eigenfunction transform - a unitary operator that diagonalizes $H$ via suitably normalized (not necessarily square-integrable) eigenfunctions.

For $N>1$ and $U=0$ one need only work with tensor products to obtain the corresponding quantities. The resulting transform amounts to Fourier transformation in $N$ variables,

$$
(\mathcal{E} \phi)(x)=(2 \pi \hbar)^{-N / 2} \int d p \exp \left(i p \cdot \frac{x}{\hbar}\right) \phi(p) .
$$

(From now on, we omit the integration region when it equals $\mathbb{R}^{k}$ for some $k \in \mathbb{N}$.) It diagonalizes not only PDOs with constant coefficients, but also constant coefficient A $\Delta$ Os. Indeed, from (4.3) one gets

$$
\exp \left(a \hat{p}_{j}\right) \exp \left(i p \cdot \frac{x}{\hbar}\right)=\exp \left(a p_{j}\right) \exp \left(i p \cdot \frac{x}{\hbar}\right) \text {. }
$$

Now functions of the form (4.7) with $\phi \in C_{0}^{\infty}\left(\mathbb{R}^{N}\right)$ extend to entire functions in $x_{1}, \ldots, x_{N}$, and so the quantum versions

$$
S_{k} \equiv \sum_{|I|= \pm k} \exp \left( \pm \beta \hat{p}_{I}\right), \quad \pm k=1, \ldots, N
$$

of the functions (3.19) with $f=f_{T}=1$ satisfy

$\left(S_{k} \mathcal{E} \phi\right)(x)=(2 \pi \hbar)^{-N / 2} \int d p \exp \left(i p \cdot \frac{x}{\hbar}\right) e_{k}(p) \phi(p), \phi \in C_{0}^{\infty}\left(\mathbb{R}^{N}\right)$

with

$$
e_{k}(p) \equiv \sum_{|I|= \pm k} \exp \left( \pm \beta p_{I}\right)
$$

The above is quite elementary and well known, but it differs in spirit and notation from the Dirac approach employed in most quantum mechanics textbooks. We shall have more to say about these differences shortly, but 
first we would like to introduce and discuss the notion of Hilbert integrability. Roughly speaking, we shall say that commuting PDOs and $\mathrm{A} \Delta \mathrm{O}$ s (of the above-defined integrable kind) are Hilbert integrable when they can be simultaneously diagonalized as real-valued multiplication operators.

To be specific, let us first assume that $I_{1}=H(x, \hat{p}), x \in G$, is an integrable PDO with commuting PDOs $I_{2}(x, \hat{p}), \ldots, I_{N}(x, \hat{p})$. Then we call $H$ Hilbert integrable when there exist joint eigenfunctions

$$
I_{k} E(x, p)=M_{k}(p) E(x, p), \quad p \in \Lambda, k=1, \ldots, N,
$$

(with $\Lambda$ a subset of $\mathbb{R}^{N}$ and $M_{k}$ a real-valued continuous function) and a unitary operator

$$
\begin{gathered}
\mathcal{E}: \hat{\mathcal{H}} \equiv L^{2}(\Lambda, \mu) \rightarrow \mathcal{H} \equiv L^{2}(G, d x), \\
\phi(p) \mapsto \int_{\Lambda} d \mu(p) E(x, p) \phi(p),
\end{gathered}
$$

where $\mu$ is a measure on $\Lambda$. Similarly, starting from an integrable quantization $S_{ \pm 1}, \ldots, S_{ \pm N}$ of the type I-VI Hamiltonians (3.19) (as defined above), we shall say that the commuting $\mathrm{A} \Delta \mathrm{O}$ s are Hilbert integrable when there exist joint eigenfunctions

$$
S_{k} E(x, p)=M_{k}(p) E(x, p), \quad p \in \Lambda, \pm k=1, \ldots, N,
$$

(with $\Lambda \subset \mathbb{R}^{N}$, and $M_{k}$ real-valued and continuous) and a unitary of the form (4.12). In either case, we define the commuting PDOs/A $\Delta$ Os as commuting Hilbert space operators by pulling back the multiplication operators $M_{k}$ to $\mathcal{H}$ via $\mathcal{E}$.

Starting from integrable PDOs $I_{1}, \ldots, I_{N}$ or A $\Delta$ Os $S_{ \pm 1}, \ldots, S_{ \pm N}$, there is no simple method to establish whether they are Hilbert integrable-and if they are, the unitary $\mathcal{E}$ and the resulting commuting operators on $\mathcal{H}$ need not be unique, even in the free case. In this connection, a key question is: which solutions to the above time-independent Schrödinger equations are relevant in the Hilbert space context?

For PDOs much is known about this question, whereas for $\mathrm{A} \Delta \mathrm{O}$ s of the type occurring in our models very little is known. The different character of the two classes of operators can already be gleaned from simple operators $H$ on $L^{2}(\mathbb{R})$. Taking $H=\hat{p}$, the solution of (4.5) (unique up to a multiplicative constant) contributes to the eigenfunction transform (4.6) for all $E=p \epsilon$ $\mathbb{R}$. For $H=\hat{p}^{2}$, however, we should restrict attention to $E=p^{2}>0$, anc then we need the two linearly independent solutions $\exp \left( \pm i E^{1 / 2} x / \hbar\right)$ fo: the eigenfunction transform, which is again given by (4.6). (The constan solution for $E=0$ is bounded, too, but its contribution to (4.6) may b ignored.) Now take $H=\hat{p}^{2 k}, k>1$. Then we need again $E=p^{2 k}>1$ to get the two bounded solutions making up the diagonalizing transforn (4.6), but now we have to discard $2 k-2$ linearly independent unbounder solutions for all $E>0$. 
For the $\mathrm{A} \Delta \mathrm{O} H=\exp (a \hat{p})$ the situation is essentially different, though. Of course, the plane wave $\exp (i p x / \hbar)$ is the only solution to (4.5) for $E=$ $e^{a p}>0$ that is needed to construct a unitary eigenfunction transform (4.6). Here, however, one is throwing away an infinite-dimensional vector space of bounded solutions to (4.5)! Indeed, one can multiply the plane wave by any function that is entire (say), has period $i a \hbar$, and is bounded on $\mathbb{R}$. The functions $\exp (i q \operatorname{ch}(2 \pi x / a \hbar+r)), q, r \in \mathbb{R}$, have all of these properties, for example. One can also construct bound states by taking instead $q \in i(0, \infty)$, or allow meromorphic multipliers of the form $\operatorname{ch}(2 \pi x / a \hbar+i r) /$ c.c.,$r \in \mathbb{R}$.

A closely related phenomenon can be illustrated by the type II A $\Delta$ Os. As we will see in Section 4.3 , their coefficients have period $2 i \pi / \mu$ in $x_{1}$, $\ldots, x_{N}$, just as the classical functions (3.19). Therefore, they commute with the free $\mathrm{A} \Delta \mathrm{O}$ s

$$
\tilde{S}_{k} \equiv \sum_{|I|= \pm k} \exp \left( \pm 2 \pi(\mu \hbar)^{-1} \hat{p}_{I}\right), \quad \pm k=1, \ldots, N .
$$

(Worse yet, they commute with any A $\Delta \mathrm{O}$ obtained from $\widetilde{S}_{k}$ via multiplication of the shift monomials by arbitrary $x$-dependent coefficients, as long as the latter have period $i \beta \hbar$ in $x_{1}, \ldots, x_{N}$ !) But the plane waves that diagonalize the $\widetilde{S}_{k}$ are not in any sense eigenfunctions of the type II A $\Delta$ Os (unless the latter have constant coefficients, too).

From these simple observations it can already be seen that it is quite difficult to interpret the relevant $\mathrm{A} \Delta \mathrm{O}$ s as well-defined commuting Hilbert space operators. We shall return to this problem in Section 6. This subsection will be concluded by sketching some material from Hilbert space theory, which puts the above in its proper mathematical context. (The notions to be introduced will reappear in Section 6.) Before doing so, we insert a sociological aside.

All of what follows can already be found in von Neumann's classic [41], which was written more than sixty years ago. The subject matter summarized below should have become bread and butter for theoretical physicists, but is actually still widely ignored-if not taboo. Indeed, almost all of the standard textbooks on quantum mechanics still contain a brand of Hilbert space theory that is considered antediluvian by functional analysts-to put it kindly. Up-to-date accounts oriented toward theoretical physics can be found for instance in Refs. 13, 42-45, and with such lucid and elegant sources available, one need not spend undue effort in learning the basics. From a purely pragmatic standpoint, too, the analyst's mathematical framework for quantum mechanics is most useful, as it pins down the essential difficulties and prevents tilting at windmills.

With the preaching and advertising out of the way, let us turn to less contentious matters. First of all, it should be recalled that quantum mechanical Hamiltonians are typically unbounded operators, whose definition must include the specification of a dense subspace of the Hilbert space as a 
domain to act on. (As a consequence of unboundedness, they do not admit a continuous linear extension to all of Hilbert space.) Now for any (linear) operator $H$ defined on a dense domain $\mathcal{D}$ in the (separable, complex) Hilbert space $\mathcal{H}$ one can define the adjoint $H^{*}$ on a definition domain $\mathcal{D}^{*}$, as follows: A vector $\phi \in \mathcal{H}$ belongs to $\mathcal{D}^{*}$ iff there exists a vector $\phi^{*} \in \mathcal{H}$ such that

$$
\left(\phi^{*}, \psi\right)=(\phi, H \psi), \quad \forall \psi \in \mathcal{D} .
$$

Then the action of $H^{*}$ on $\phi$ is defined by

$$
H^{*} \phi \equiv \phi^{*} \text {. }
$$

The definition domain $\mathcal{D}^{*}$ of the adjoint $H^{*}$ need not be dense in $\mathcal{H}$; in fact, it may even consist solely of the zero vector. From now on we assume that the operator $H$ is symmetric, i.e., that we have

$$
(\phi, H \psi)=(H \phi, \psi), \quad \forall \phi, \psi \in \mathcal{D} .
$$

Then $\mathcal{D}^{*}$ obviously contains $\mathcal{D}$, and so is dense in $\mathcal{H}$; moreover, on $\mathcal{D}$ the adjoint $H^{*}$ coincides with $H$. For instance, the type I-VI defining Hamiltonians (4.2) are symmetric on the dense subspace $C_{0}^{\infty}(G) \subset L^{2}(G)$-as is readily checked.

Next, we turn to an example that we will use to motivate the notions of self-adjointness and self-adjoint extensions. Specifically, we start from

$$
\mathcal{H}=L^{2}([0, \pi]), \quad \mathcal{D}=C_{0}^{\infty}((0, \pi)), \quad H=-\frac{d^{2}}{d x^{2}} .
$$

(This setting actually arises for the center-of-mass, $g=0, N=2$, type III and IV Hamiltonians; we are putting $\hbar=1$ for the remainder of this subsection.) Obviously, $H$ is symmetric. Here, $\mathcal{D}^{*}$ contains the space $C^{2}([0, \pi])$, and on this space $H^{*}$ acts again as $-d^{2} / d x^{2}$. (Use the above definition and integration by parts to check this.) In particular, the functions $\psi_{p} \equiv$ $\exp (i x p), p \in \mathbb{C}$, belong to $C^{2}([0, \pi])$, and one has $H^{*} \psi_{p}=p^{2} \psi_{p}$. Therefore, the adjoint has spectrum $\mathbb{C}$.

Now this is bad news for physics, since the spectrum should correspond to the physically measurable energies of the system-which are real. This is why one should work with Hamiltonians that are not just symmetric, but self-adjoint (s.a.) or at least essentially self-adjoint (e.s.a.). By definition, a symmetric $H$ is s.a. iff $\mathcal{D}^{*}$ equals $\mathcal{D}$, and is e.s.a. iff $H^{*}$ is s.a. If $H$ is symmetric, but not e.s.a., one should try and extend $H$ to an operator $H_{e}$ on a definition domain $\mathcal{D}_{e}$ satisfying $\mathcal{D} \subset \mathcal{D}_{e} \subset \mathcal{D}^{*}$, such that $H_{e}$ is s.a.

A symmetric operator need not have any s.a. extensions, but when it commutes with a conjugation (i.e., an anti-unitary with square the identity), then it does admit s.a. extensions. When $H$ is e.s.a., it also admits s.a. extension, viz., to the operator $H^{*}$; in this case the s.a. extension is unique. 
Returning to our example (4.15), we see that $H$ commutes with complex conjugation, so it admits s.a. extensions. These are not unique, however, so that $H$ is not e.s.a. Indeed, three distinct s.a. extensions of $H$ are well known: We can enlarge the definition domain by allowing smooth functions with $\psi(0)=\psi(\pi), \psi^{\prime}(0)=\psi^{\prime}(\pi)$ (periodic boundary conditions), or with $\psi(0)=\psi(\pi)=0$ (Dirichlet b.c.), or with $\psi^{\prime}(0)=\psi^{\prime}(\pi)=0$ (Neumann b.c.). These extensions yield e.s.a. operators with a complete set of bound state eigenfunctions, namely, linear combinations of $\exp ( \pm i p x)$ for appropriate $p \in[0, \infty)$. In this way one obtains three distinct real point spectra. Thus, the physics depends on what s.a. extension is chosen.

To summarize, one should insist on quantum-mechanical Hamiltonians being s.a. or at least e.s.a., so as to ensure reality of the spectrum. Selfadjointness is also sufficient for applicability of the spectral theorem, to which we now turn. This theorem can be presented in several guises (cf. Ref. 42, Chapters VII, VIII), but in our context of quantum-integrable systems one of these is particularly useful. Crudely speaking, it says that any s.a. operator $H$ with dense definition domain $\mathcal{D}$ in an abstract Hilbert space $\mathcal{H}$ can be unitarily transformed to a real-valued multiplication operator on a concrete space of square-integrable functions. More precisely, the multiplication operators arising in this way can be defined as follows. Let $\widehat{\mathcal{H}}$ be the Hilbert space

$$
\hat{\mathcal{H}} \equiv \bigoplus_{j=1}^{m} L^{2}\left(\mathbb{R}, \mu_{j}\right), \quad m \leq \infty,
$$

where $\mu_{1}, \mu_{2}, \ldots$ are measures on the real line. Then the operator $M: \widehat{\mathcal{D}} \rightarrow$ $\widehat{\mathcal{H}}$ with domain

$$
\widehat{\mathcal{D}} \equiv\left\{\psi \in \widehat{\mathcal{H}} \mid\left(E \psi_{1}(E), E \psi_{2}(E), \ldots\right) \in \widehat{\mathcal{H}}\right\}
$$

and action

$$
(M \psi)_{j}(E) \equiv E \psi_{j}(E), \quad \psi \in \widehat{\mathcal{D}}, j=1,2, \ldots,
$$

is s.a. Now the spectral theorem says that for any s.a. operator $H: \mathcal{D} \rightarrow \mathcal{H}$ there exists a space $\widehat{\mathcal{H}}$ of the above form and a unitary $\mathcal{E}$ from $\widehat{\mathcal{H}}$ onto $\mathcal{H}$ satisfying

$$
\mathcal{E}(\hat{\mathcal{D}})=\mathcal{D}, \quad H \mathcal{E}=\mathcal{E} M .
$$

Thus, $H$ is diagonalized by the unitary $\mathcal{E}$.

Of course, the choice of spectral representation space $\widehat{\mathcal{H}}$ is highly nonunique: parts of the measures in (4.16) can be reshuffled among the summands, and the normalization of the measures is not fixed either. But the closure of the union of the supports of the measures is unique: It is the spectrum of $H$. More generally, the measures encode all spectral properties 
of $H$. For example, whenever one of the measures $\mu_{j}$ assigns nonzero weight to a point $E_{0} \in \mathbb{R}, H$ has a bound state with eigenvalue $E_{0}$; continuous parts of the spectrum correspond to continuous parts of the measures; the spectral multiplicity of $E \in \mathbb{R}$ equals the limit of the number of measures assigning nonzero weight to the interval $(E-\epsilon, E+\epsilon)$ as $\epsilon \downarrow 0$, etc.

As a matter of fact, it is often more convenient to diagonalize $H$ as a nonlinear function of a spectral variable; we have already seen several concretizations of this more general form of the spectral theorem. If need be, one can easily convert this to the previous form. For instance, the Fourier transformation (4.6) diagonalizes all operators $\hat{p}^{l}, l \in \mathbb{N}^{*}$, and $\exp (a \hat{p})$, $a \in \mathbb{R}^{*}$, simultaneously; then one should transform to $L^{2}([0, \infty), d E) \oplus$ $L^{2}([0, \infty), d E)$ for even $l$ and to $L^{2}(\mathbb{R}, d E)$ otherwise.

The spectral theorem makes it possible to define and work with functions of $H$. In particular, one way to obtain the quantum time evolution is by pulling back the unitary multiplication operator $\exp (-i t E)$ on $\hat{\mathcal{H}}$ :

$$
\exp (-i t H)=\mathcal{E} \exp (-i t E) \mathcal{E}^{-1}
$$

More generally, one can define bounded functions of $H$, and these functions form an Abelian algebra. Whenever one can choose $m=1$ in (4.16) (so that the (global) spectral multiplicity of $H$ equals 1 ), this algebra is maximal Abelian: It cannot be enlarged without losing the commutativity property.

The spectral theorem has a generalization to several commuting s.a. operators $H_{1}, H_{2}, \ldots, H_{N}$. Here, commuting means by definition that the corresponding evolutions $\exp \left(-i t_{j} H_{j}\right), j=1, \ldots, N$, commute. (For bounded $H_{1}, \ldots, H_{N}$, this is equivalent to $\left[H_{k}, H_{l}\right]=0$. But for unbounded operators, the commutator need not be densely defined, and even if it is and vanishes, this does not entail that the evolutions commute.) Again, it is often simpler to transform to a representation where $H_{1}, \ldots, H_{N}$ become nonlinear functions of several variables (instead of multiplication by $E_{1}$, $\ldots, E_{N}$, respectively). The free A $\Delta O$ s (4.8) are a case in point; cf. (4.9), (4.10). Note that they all have spectrum $[0, \infty)$ with infinite multiplicity. The Abelian algebra generated by the bounded functions of $S_{1}, \ldots, S_{N}$ (say) is, however, almost multiplicity-free (maximal): Its commutant is generated by the permutation operators

$$
\left(P_{\sigma} \psi\right)(x) \equiv \psi\left(\sigma^{-1}(x)\right), \quad \sigma \in S_{N}, \psi \in L^{2}\left(\mathbb{R}^{N}, d x\right)
$$

where $\tau(x)$ stands for $\left(x_{\tau(1)}, \ldots, x_{\tau(N)}\right)$.

\subsection{The Nonrelativistic Case: Commuting PDOs}

The quantization prescription (4.1) applied to the Hamiltonian (2.13) yields the PDO 


$$
H=\frac{1}{2} \sum_{j=1}^{N} \hat{p}_{j}^{2}+g^{2} \sum_{\substack{j, k=1 \\ j<k}}^{N} V\left(x_{j}-x_{k}\right), \quad g>0 .
$$

Specializing $V(x)$ to the type I-IV cases (2.18)-(2.21), the first question to answer is now, whether there exist $N$ independent PDOs (including $H$ ) that commute pairwise. Equivalently, one should try and establish whether $H$ is an integrable PDO; cf. the first few paragraphs of Section 4.1.

There are two sets of obvious candidates for these commuting PDOs. Clearly, the quantization $P \equiv \sum_{j} \hat{p}_{j}$ of the first power trace $I_{1} /$ symmetric function $S_{1}$ of the respective Lax matrices commutes with $H$; also, $H$ is the unambiguous quantization of $I_{2}$ (or equivalently $\frac{1}{2} S_{1}^{2}-S_{2}$ ) up to an (irrelevant) $\lambda$-dependent constant for type IV. For $N>2$ one can now try to continue either with $I_{k}(x, \hat{p}), k=3, \ldots, N$, or with $S_{k}(x, \hat{p}), k=3$, $\ldots, N$. But for the power traces ordering problems arise: in summands contributing to $I_{k}(x, p)$ for $k>2$ the quantities $x_{j}$ and $p_{j}$ can occur simultaneously, so the ordering is ambiguous. By contrast, whenever $p_{j}$ occurs in a monomial contributing to some principal minor of $L(x, p)$, the position $x_{j}$ cannot occur, so that the expression $S_{k}(x, \hat{p})$ yields an unambiguous PDO for all $k \in\{1, \ldots, N\}$. Thus one need only show that $S_{1}(\hat{p}), \ldots, S_{N}(x, \hat{p})$ commute. (If so, one can define $I_{k}(x, \hat{p}), k>2$, unambiguously via the Newton identities (2.34).)

We have no doubt that these PDOs indeed commute. But as we have learned, there appears to be no complete proof in the published literature. In Ref. 2, Olshanetsky and Perelomov do present a proof, but upon scrutiny this proof turns out to be incomplete on two counts-as we shall detail shortly.

Fortunately, there are complete proofs that $H$ (4.18) is integrable for all of the systems of type I-VI; only the connection between the higherorder commuting PDOs and the quantized $S_{k}(L)$ has not been completely clarified yet. As we see it now, the first complete proof for systems of type I-III was given by Heckman [15], Opdam [46, 47], and Heckman and Opdam [48], and for systems of type IV by Oshima and H. Sekiguchi [49]. In our survey [5] we presented independent proofs of integrability for the nonrelativistic quantum systems of type I, II, III, V, and VI-as a corollary of integrability at the relativistic level, proved first in Ref. 4. We shall return to the latter strategy in Section 4.3 .

We proceed by discussing the partial proof in Ref. 2 and the complete proof of Ref. 49. The starting point of Ref. 2 is a classical Hamiltonian of the form

$$
J_{N}(x, p) \equiv \exp \left(\sum_{\substack{j, k=1 \\ j<k}}^{N} h\left(x_{j}-x_{k}\right) \partial_{p_{j}} \partial_{p_{k}}\right) p_{1} \cdots p_{N} .
$$

Expanding the exponential, this can be written 


$$
\begin{aligned}
J_{N}=\sum_{l=0}^{[N / 2]} \frac{1}{2^{l} l !(N-2 l) !} \\
\quad \times \sum_{\sigma \in S_{N}} \sigma\left(h\left(x_{1}-x_{2}\right) \cdots h\left(x_{2 l-1}-x_{2 l}\right) p_{2 l+1} \cdots p_{N}\right)
\end{aligned}
$$

Next, defining recursively

$$
J_{k-1} \equiv \frac{\left\{\sum_{j=1}^{N} x_{j}, J_{k}\right\}}{N-k+1}, \quad k=N, N-1, \ldots, 1
$$

and using (4.20), one obtains

$$
\begin{aligned}
J_{k}=\frac{1}{(N-k) !} & \sum_{l=0}^{[k / 2]} \frac{1}{2^{l} l !(k-2 l) !} \\
& \times \sum_{\sigma \in S_{N}} \sigma\left(h\left(x_{1}-x_{2}\right) \cdots h\left(x_{2 l-1}-x_{2 l}\right) p_{2 l+1} \cdots p_{k}\right) .
\end{aligned}
$$

In particular, this yields

$$
\begin{aligned}
J_{0}=1, \quad J_{1} & =\sum_{j} p_{j}, \quad J_{2}=\sum_{j<k} p_{j} p_{k}+\sum_{j<k} h\left(x_{j}-x_{k}\right), \\
J_{3} & =\sum_{i<j<k} p_{i} p_{j} p_{k}+\sum_{\substack{j<k \\
i \neq j, k}} h\left(x_{j}-x_{k}\right) p_{i},
\end{aligned}
$$

so that

$$
\frac{1}{2} J_{1}^{2}-J_{2}=\frac{1}{2} \sum_{j} p_{j}^{2}-\sum_{j<k} h\left(x_{j}-x_{k}\right) \equiv H .
$$

Hence, the choice

$$
h(x)=-g^{2} \mathcal{P}(x)-C
$$

yields the classical type IV Hamiltonian (2.13), (2.21) when one takes $C=0$. More generally, one easily checks

$$
J_{k}(h+C)=\frac{1}{(N-k) !} \sum_{j=0}^{[k / 2]} \frac{C^{j}}{2^{j} j !}(N-k+2 j) ! J_{k-2 j}(h) .
$$

In words, shifting $h$ by a constant amounts to a linear reshuffling of $J_{1}$, $\ldots, J_{N}$.

Evidently, all of these quantities have unambiguous quantizations, and the proof of Ref. 2 is concerned with the commutativity of $J_{1}, \ldots, J_{N}$ given by the quantizations of (4.19), (4.21). Their proof uses classical input, which 
we now sketch. The Hamiltonian $J_{N}(x, p)(4.19)$ was introduced by Sawada and Kotera [50] for the type I case, where $h(x)=-g^{2} / x^{2}$. They showed that $J_{N}$ Poisson commutes with $H(4.23)$ and observed that this entails that the Hamiltonians $J_{k}$ defined via (4.21) commute with $H$, as well. (Indeed, this easily follows from the Jacobi identity [50].) Subsequently, Wojciechowski [51] generalized $J_{N}(4.19)$ to the type IV case and showed that when $J_{k}$ is defined via the recurrence relation (4.21), then $J_{1}, \ldots, J_{N}$ are in involution.

Now Olshanetsky and Perelomov do prove that the quantum versions of $H$ and $J_{N}$ commute as well, by showing that the additional terms in the commutator (compared to the terms in the Poisson bracket) sum to zero; cf. Ref. 2, p. 336. Then it follows just as in the classical case that $H$ also commutes with $J_{N-1}, \ldots, J_{1}$. But in Ref. 2 it is not proved (or even made plausible) that the additional terms in arbitrary commutators $\left[J_{k}, J_{l}\right]$ sum to zero.

The latter gap is closed in the work by Oshima and Sekiguchi [49]. Specifically, they prove (among other things) that the quantizations of $J_{k}(4.22)$ with (4.24) in force commute without using any classical input (so that involutivity of $J_{1}(x, p), \ldots, J_{N}(x, p)$ follows as a corollary). They also prove a most useful uniqueness result, which we shall have occasion to invoke in Section 4.3. More precisely, we only need a slightly weaker version of Theorem 5.2 in Ref. [49], which we now state.

Suppose $G_{k}(x, \hat{p}), k=1, \ldots, N, x \in \mathbb{R}^{N}$, are $N$ commuting PDOs with meromorphic dependence on $x_{1}, \ldots, x_{N}$. Suppose that the PDOs are invariant under arbitrary permutations in $S_{N}$ and that they are of the form

$$
\begin{gathered}
G_{1}=\sum_{j} \hat{p}_{j}, \quad G_{2}=\sum_{j<k} \hat{p}_{j} \hat{p}_{k}-g_{2}(x), \\
G_{k}=\sum_{i_{1}<\cdots<i_{k}} \hat{p}_{i_{1}} \cdots \hat{p}_{i_{k}}-g_{k}(x, \hat{p}), k=3, \ldots, N,
\end{gathered}
$$

with $g_{k}$ of degree $<k-1$ in $\hat{p}$. Then the commutative PDO algebra generated by $G_{1}, \ldots, G_{N}$ coincides with the algebra generated by the above $J_{1}, \ldots, J_{N}$. In particular, one must have

$$
g_{2}(x)=C_{1} \sum_{j<k} \mathcal{P}\left(x_{j}-x_{k} ; \omega, \omega^{\prime}\right)+C_{2}
$$

Thus far, we have restricted ourselves to the type I-IV systems. Let us now turn to the Toda systems. Choosing

$$
h(x)=-g^{2}(\mathcal{P}(x)-\mathcal{P}(\lambda))=-g^{2} \frac{s(\lambda+x) s(\lambda-x)}{s^{2}(\lambda) s^{2}(x)}, \quad \omega^{\prime} \equiv \frac{i \pi}{\mu},
$$

with $\lambda \equiv \omega+i \pi / \mu$, and substituting (2.66) and (2.68) in $J_{N}$ (4.19), it follows from previous calculations that the limit $\omega \rightarrow \infty$ exists and yields 
(with the convention (3.15) in effect)

$$
J_{N}=\exp \left(-a^{2} \sum_{j=1}^{N} \exp \mu\left(x_{j}-x_{j-1}\right) \partial_{p_{j}} \partial_{p_{j-1}}\right) p_{1} \cdots p_{N}
$$

The limit $\omega \rightarrow \infty$ can also be taken in the recurrence relation (4.21), and so one winds up with commuting PDOs $J_{1}(x, \hat{p}), \ldots, J_{N}(x, \hat{p})$. In particular, one gets in this way

$$
J_{1}=\sum_{j} \hat{p}_{j}, \quad J_{2}=\sum_{j<k} \hat{p}_{j} \hat{p}_{k}-a^{2} \sum_{j=1}^{N} \exp \mu\left(x_{j}-x_{j-1}\right),
$$

so that

$$
\frac{1}{2} J_{1}^{2}-J_{2}=\sum_{j=1}^{N}\left(\frac{1}{2} \hat{p}_{j}^{2}+a^{2} \exp \mu\left(x_{j}-x_{j-1}\right)\right)=H(\mathrm{~V}) .
$$

Substituting (2.60) and taking $a \rightarrow 0$ now yields the nonperiodic Toda analogs, in particular

$$
J_{N}=\exp \left(-\sum_{j=2}^{N} \exp \mu\left(x_{j}-x_{j-1}\right) \partial_{p_{j}} \partial_{p_{j-1}}\right) p_{1} \ldots p_{N}
$$

Alternatively, one can obtain the commuting type VI PDOs from the hyperbolic PDOs by substituting (2.69) and (2.70) in $J_{1}, \ldots, J_{N}$ with

$$
h(x)=-\frac{g^{2} \mu^{2}}{4 \operatorname{sh}^{2}(\mu x / 2)}
$$

and taking $\epsilon \rightarrow 0$. To complete the connection diagram (2.62) at the quantum nonrelativistic level, it remains to specify the direct IV $\rightarrow$ VI transition. To this end we use once more (4.28), but now with $\lambda \equiv \omega$. Substituting then (2.72) and (2.73) in $J_{N}(4.19)$, it follows that $J_{N}$ converges to (4.30); taking successively $k=N-1, \ldots, 1$, one deduces that $J_{k}$ converges to the type VI $J_{k}$.

To conclude this subsection, we elaborate on the relation between $J_{1}$, $\ldots, J_{N}$ and the symmetric functions $S_{1}, \ldots, S_{N}$ of the type II Lax matrix (2.35) (and its type I and III versions), its $\lambda$-dependent type IV generalization (2.51), and the type V and VI Lax matrices (2.57), (2.59), respectively. First, it should be noted that whenever $J_{N}$ and $S_{N}$ are equal, equality of $J_{k}$ and $S_{k}$ for all $k \in\{1, \ldots, N\}$ follows. Indeed, the functions $S_{1}, \ldots, S_{N}$ also satisfy the recurrence relation (4.21). (The coefficient of $p_{j}$ in $S_{k}$ equals the sum of all principal minors of order $k-1$ not containing the index $j$. Each such minor does not contain $N-k+1$ indices, so it occurs $N-k+1$ times in the Poisson bracket at the right-hand side.) 
Consequently, if $J_{N}(x, p)$ were equal to $S_{N}(x, p)$, the connection diagram (2.62) for the quantum versions would be immediate from the classical connections detailed in Section 2.3. Now when we choose $h(x)$ equal to (4.28), we must choose the same $\lambda$ in (2.51) to obtain equality for $N=2$. But then we do not get equality for $N=3$, unless

$$
\lambda \equiv \omega_{i}, \quad i=1,2,3, \quad \omega_{1} \equiv \omega, \quad \omega_{2} \equiv-\omega-\omega^{\prime}, \quad \omega_{3} \equiv \omega^{\prime}
$$

(modulo the period lattice), and even for these three choices, it is quite likely that one does not get equality for arbitrary $N$.

Turning now to the assertions of Ref. 1, it is claimed on p. 326 that their $J_{N}$ (eq. (4.8) in l.c.) equals the determinant of Lax matrices specified on pp. 322-323. However, no proof of this claim is presented. Instead, the reader is referred to the papers by Sawada and Kotera [50] and Wojciechowski [51] already mentioned. But in these papers equality of $J_{N}$ to a determinant is neither proved nor claimed to be valid.

Translated into our notation, the above claim says in particular that when one takes $h(x)$ in $J_{N}(4.19)$ equal to (4.31), then $J_{N}$ equals $S_{N}(L)$, with $L$ given by (2.35). Undoubtedly this is true, and we have checked equality for $N \leq 4$. However, we are not aware of a complete proof. Changing $1 /$ sh to coth in (4.31) and in (2.35), Ref. 1 claims once more equality of $J_{N}$ and $S_{N}$. (This choice amounts to the $\lambda=i \pi / \mu$ specialization of the $\lambda$-dependent type II Lax matrix, i.e., (2.51) with $\omega=\infty$.) This claim is false: $J_{4}$ and $S_{4}$ differ by a constant, so $J_{N}$ and $S_{N}$ differ for all $N \geq 5$, too.

In the elliptic case Ref. 1 allows three choices for $h$ and the Lax matrix. These choices amount to the above choices (4.32). Again, the claim that $J_{N}$ equals $S_{N}$ is incorrect in general: $J_{4}$ and $S_{4}$ differ by a constant that depends on the choice, so that nontrivial differences for $N \geq 5$ result. We conjecture that the correct result for arbitrary $\lambda \in \mathbb{C}$ in (2.51) reads

$$
S_{N}(\lambda)=J_{N}(h)+\sum_{k=0}^{N-2} c_{k, N}(\lambda) J_{k}(h), \quad h(x)=-g^{2} \mathcal{P}(x)
$$

where $J_{1}(h), \ldots, J_{N}(h)$ are given by (4.22). (We have checked (4.33) for $N \leq 3$.) For the periodic Toda case we conjecture that

$$
S_{N}(w)=J_{N}+(i a)^{N}\left(w^{-1}+(-)^{N} w\right)
$$

with $S_{N}(w)$ the determinant of (2.57) and $J_{N}$ given by (4.29). If so, equality of $S_{k}$ and $J_{k}$ for $k<N$ and equality of the type VI $S_{k}$ and $J_{k}$ for $k \leq N$ would follow, of course.

\subsection{The Relativistic Case: Commuting A $\Delta O s$}

As already discussed in Section 4.1, we run into ordering problems when we perform the canonical quantization (4.1) in the Poisson commuting Hamil- 
tonians $S_{k}(x, p)(3.19), \pm k \in\{1, \ldots, N-1\}$. More precisely, in writing down $S_{k}(x, p)$, we have automatically opted for an ordering, but for this ordering the prescription (4.1) does not necessarily yield commuting A $\Delta O$ s.

As a model for the ambiguity at hand, let us look at a Hamiltonian of the form $h(x, p) \equiv e^{p} f(x)$. Writing it as $f(x) e^{p}$ yields a different $\mathrm{A} \Delta \mathrm{O}$ upon quantization (unless $f(x)$ happens to have period $i \hbar$ ). As it turns out, both of these orderings in (3.19) give rise to noncommuting $\mathrm{A} \Delta \mathrm{OS}$. (Taking $N>2$ and generic $f, f_{T}$.) Now for these two choices the resulting $\mathrm{A} \Delta \mathrm{Os}$ are not even formally symmetric, so one can try next the orderings symbolized by $f(x)^{1 / 2} e^{p} f(x)^{1 / 2}$ and $e^{p / 2} f(x) e^{p / 2}$ (which do yield formally symmetric $\mathrm{A} \triangle \mathrm{Os}$ ). Again, these choices spoil commutativity, though. At this point it should be emphasized that no general results are known from which an ordering choice preserving commutativity would follow.

Such a choice does exist, however. Specializing first to the type I case, where $f(x)=\left(1+\beta^{2} g^{2} / x^{2}\right)^{1 / 2}$ (with $f(x)>0$ for $x \in \mathbb{R}^{*}$ ), it can be symbolized by writing the model Hamiltonian $h(x, p)$ as $f_{-}(x) e^{p} f_{+}(x)$, where $f_{ \pm}(x) \equiv(1 \pm i \beta g / x)^{1 / 2}$. Recalling $\beta, g>0$, the square-root branches may and will be fixed by requiring $f_{ \pm}(x) \rightarrow 1$ for $g \downarrow 0$. (To be quite precise, we require this for $x \in \mathbb{R}^{*}$. Since the $x$-shifts involved are in the imaginary direction, the branch points off the real axis are not encountered.)

We now turn to the corresponding ordering choice for the type IV Hamiltonians: It is obtained by choosing

$$
f_{ \pm}(x) \equiv\left(\frac{s(x \pm i \beta g)}{s(x)}\right)^{1 / 2}, \quad g \downarrow 0 \Longrightarrow f_{ \pm}(x) \rightarrow 1 .
$$

Written out, the corresponding $A \Delta O$ s read

$$
\begin{aligned}
S_{ \pm k}(x, \hat{p}) \equiv \sum_{\substack{I \subset\{1, \ldots, N\} \\
|I|=k}} \prod_{\substack{i \in I \\
j \notin I}} f_{\mp}\left(x_{i}-x_{j}\right) \\
\times \exp \left( \pm \beta \sum_{i \in I} \hat{p}_{i}\right) \prod_{\substack{i \in I \\
j \notin I}} f_{ \pm}\left(x_{i}-x_{j}\right) . \quad(\mathrm{I}-\mathrm{IV})
\end{aligned}
$$

Thus, their classical symbols yield the same functions as (3.19); moreover, (3.21) holds true again. (To check this, use $f_{\delta}(-x)=f_{-\delta}(x)$.) Using (3.18), we also get $\mathrm{A} \Delta \mathrm{Os} H(x, \hat{p})$ and $P(x, \hat{p})$ belonging to the algebra generated by the $\mathrm{A} \Delta \mathrm{Os}$ (4.35). It can be shown that the commutativity of this algebra boils down to the functional equations

$$
\sum_{\substack{I \subset\{1, \ldots, N\} \\|I|=k}}\left(\prod_{\substack{i \in I \\ j \notin I}} \frac{s\left(x_{i}-x_{j}-\gamma\right) s\left(x_{i}-x_{j}+\gamma-\rho\right)}{s\left(x_{i}-x_{j}\right) s\left(x_{i}-x_{j}-\rho\right)}-(x \rightarrow-x)\right)=0
$$

which hold true for all $N>1, k \in\{1, \ldots, N\}, x \in \mathbb{C}^{N}, \gamma, \rho \in \mathbb{C}$. These results can be found in Ref. 4, with $s(x)$ replaced by the Weierstrass $\sigma$ function; in view of the relation (2.50), this difference is inconsequential. 
The functional equations (4.36) encapsulate the integrability of all of the models considered in these lectures. We continue by elaborating on this assertion. First, dividing by $\rho$ and sending $\rho$ to 0 , one obtains the functional equations

$$
\sum_{\substack{I \subset\{1, \ldots, N\} \\|I|=k}} \sum_{i \in I} \partial_{i} \prod_{\substack{i \in I \\ j \notin I}} \frac{s\left(x_{i}-x_{j}-\gamma\right) s\left(x_{i}-x_{j}+\gamma\right)}{s\left(x_{i}-x_{j}\right)^{2}}=0 .
$$

These amount to the functional equations (3.20) that express involutivity of the classical Hamiltonians $S_{1}, \ldots, S_{N}$ [3]. Now we have shown in Section 3 that the latter commutativity result entails integrability for all of the relativistic and nonrelativistic classical systems of type I-Vi. Thus, it remains to explain how (4.36) entails integrability for their quantum versions.

We begin with the relativistic level. Of course, then we need only consider the Toda systems, since the type I-III systems are included in type IV via (2.65). In fact, we are going to detail the transitions in the diagram (2.62) at the quantum relativistic level. To this end we adapt the reasoning followed at the classical level to the A $\Delta \mathrm{Os} S_{1}, \ldots, S_{N}$, rewritten as

$$
S_{l}=\sum_{\substack{I \subset\{1, \ldots, N\} \\|I|=l}} \prod_{\substack{j \in I \\ k \notin I}} f_{-}\left(x_{j}-x_{k}\right) f_{+}\left(x_{j}-x_{k}-i \hbar \beta\right) \exp \left(\beta \hat{p}_{I}\right) ;
$$

cf. (4.35). (The corresponding transitions for $S_{-1}, \ldots, S_{-N}$ can be dealt with by taking $\beta \rightarrow-\beta$.)

First, we consider the transition II $\rightarrow$ VI. Substituting (3.24) and (2.69) in $f_{-}\left(x_{j}-x_{k}\right) f_{+}\left(x_{j}-x_{k}-i \hbar \beta\right)$, we obtain a function $f_{j k}$ with limits

$$
f_{j k} \rightarrow \begin{cases}1, & |j-k|>1 \\ \left(1+\beta^{2} \exp \left[\mu\left(x_{j}-i \hbar \beta-x_{j-1}\right)\right]\right)^{1 / 2}, & k=j-1 \\ \left(1+\beta^{2} \exp \left[\mu\left(x_{j+1}-x_{j}\right)\right]\right)^{1 / 2}, & k=j+1\end{cases}
$$

for $\epsilon \rightarrow 0$. Hence, the limit of $S_{l}(4.37)$ exists and can be rewritten

$$
S_{k}=\sum_{\substack{I \subset\{1, \ldots, N\} \\|I|=k}} \prod_{\substack{j \in I \\ j+1 \notin I}} f_{T}\left(x_{j+1}-x_{j}\right) \exp \left(\beta \hat{p}_{I}\right)
$$

with the convention (3.16) in effect.

Second, we handle the IV $\rightarrow \mathrm{V}$ limit, starting again from (4.37). To this end we use the analog of the factorization (3.27) for the functions $f_{+}$and $f_{-}$, and mimic the reasoning in the classical case. Thus we introduce

$$
S_{r, k} \equiv \frac{S_{k}}{\rho(g)^{k(N-k)}}, \quad \rho(g) \equiv \exp \left(\frac{\mu \beta^{2} g(g-\hbar)}{4 \omega}\right),
$$


and substitute (2.66) and (3.29). Then the limit $\omega \rightarrow \infty$ exists and can be rewritten as (4.38), but now with (3.15) in force. (Just as in the classical case, the infinite products supply the extra boundary terms, as compared to the nonperiodic case.)

Third, the IV $\rightarrow$ VI transition can be made by substituting (2.72) and (3.31) in the A $\Delta$ Os $S_{r, k}$ and taking $\omega \rightarrow \infty$. To complete the diagram (2.62) it remains to specify the $\mathrm{V} \rightarrow \mathrm{VI}$ limit. As before, it suffices to substitute (2.60) in the type V A $\Delta \mathrm{Os} S_{k}$ (4.38) and take $a \rightarrow 0$ to obtain the type VI $S_{k}$.

It can also be shown directly that the Toda $A \Delta O$ s (4.38) commute. This can be reduced to the functional equations

$$
\begin{aligned}
\sum_{\substack{I \subset\{1, \ldots, N\} \\
|I|=k}} & \prod_{\substack{i \in I \\
i-1 \notin I}} f_{T}^{2}\left(x_{i-1}-x_{i}\right) \prod_{\substack{i \in I \\
i+1 \notin I}} f_{T}^{2}\left(x_{i}-x_{i+1}+\lambda\right) \\
= & \sum_{\substack{I \subset\{1, \ldots, N\} \\
|I|=k}} \prod_{\substack{i \in I \\
i-1 \notin I}} f_{T}^{2}\left(x_{i-1}-x_{i}+\lambda\right) \prod_{\substack{i \in I \\
i+1 \notin I}} f_{T}^{2}\left(x_{i}-x_{i+1}\right)
\end{aligned}
$$

which hold true for all $N>1, k \in\{1, \ldots, N\}, x \in \mathbb{C}^{N}, \lambda \in \mathbb{C}[37]$. (Note this yields the classical functional equations (3.20) when one divides by $\lambda$ and takes $\lambda$ to 0 .)

We now consider the nonrelativistic limit $\beta \rightarrow 0$, handling first the elliptic case. We start from commuting $\mathrm{A} \Delta \mathrm{O}$ s

$$
A_{k}(\beta) \equiv \sum_{l=0}^{k}(-)^{k+l}\left(\begin{array}{c}
N-l \\
N-k
\end{array}\right) c_{l}(\lambda, i \beta g) S_{l}, \quad k=1, \ldots, N
$$

where

$$
c_{k}(\lambda, \alpha) \equiv s(\lambda)^{-k} s(\lambda-\alpha)^{k-1} s(\lambda+(k-1) \alpha), \quad k=1, \ldots, N .
$$

The point of this definition is that the classical versions of these $A \Delta O$ s, after division by $\beta^{k}$, converge to the Hamiltonians $S_{k}\left(L_{\mathrm{nr}}\right)$ for $\beta \rightarrow 0$, where $L_{\mathrm{nr}}$ is the type IV Lax matrix (2.51). (To see this, recall (3.38), (3.39), and (3.45)-(3.48).)

Expanding $c_{l}$ and $S_{l}$ in powers of $\beta$, we now write

$$
A_{k}(\beta)=\sum_{m=0}^{\infty} A_{k, m} \beta^{m}
$$

(This expansion is meant in the sense of formal power series.) Then we calculate

$$
A_{1,0}=0, \quad A_{1,1}=\sum_{j} \hat{p}_{j}
$$




$$
\begin{gathered}
A_{2,0}=A_{2,1}=0, \\
A_{2,2}=\sum_{j<k}\left(\hat{p}_{j} \hat{p}_{k}-g(g-\hbar)\left(\mathcal{P}\left(x_{j}-x_{k}\right)+\frac{\eta}{\omega}\right)\right) \\
\quad+\frac{g^{2}}{2} N(N-1)\left(\mathcal{P}(\lambda)+\frac{\eta}{\omega}\right) .
\end{gathered}
$$

It is important to observe that this result differs from the classical expansion: In $A_{2,2}$ one has a coefficient $g(g-\hbar)$ and not $g^{2}$, since the partials in (4.35) do not commute with the $f_{+}$-factors. (Equivalently, the $\beta$ dependence of the coefficients in (4.37) differs from that of their classical versions.)

Next, we note that the integer

$$
n_{k} \equiv \min \left\{l \mid A_{k, l} \neq 0\right\}, \quad k=1, \ldots, N,
$$

equals $k$ in the classical case, and must be $\leq k$ in the quantum case, since we clearly have

$$
A_{k, k}=\sum_{i_{1}<\cdots<i_{k}} \hat{p}_{i_{1}} \cdots \hat{p}_{i_{k}}, \quad g=0 .
$$

Taking for granted that $n_{k}=k$ in the quantum case as well, it would follow that

$$
\left[A_{k, k}, A_{l, l}\right]=0, \quad k, l=1, \ldots, N,
$$

and since we have (using obvious notation)

$$
H_{\mathrm{nr}}\left(g^{2} \rightarrow g(g-\hbar)\right)=\frac{1}{2}\left(A_{1,1}\right)^{2}-A_{2,2}+\text { constant }
$$

integrability of the nonrelativistic Hamiltonian would follow.

Unfortunately, a direct proof that $n_{k}$ equals $k$ appears intractable. The problem is that one gets an avalanche of additional terms arising when partials act on $f_{+}$-factors. A priori, these extra terms might lower $n_{k}$ as compared to the classical case, and yield PDOs $A_{k, n_{k}}$ that are not independent for $k=1, \ldots, N$. Note also that the connection of $A_{k, k}$ to the quantized symmetric functions of $L_{\mathrm{nr}}$ is quite opaque due to the extra terms.

We shall now show that $n_{k}$ indeed equals $k$, by making a detour involving a function $w(\beta ; x)$ that is also an important ingredient for obtaining relativistic eigenfunctions (as we shall see in Section 6). In the process, we shall obtain a rather explicit formula for $A_{k, k}$.

The function $w$ is introduced in Ref. 31 . It is a solution to the first-order analytic difference equation

$$
w(x-i \hbar \beta)=\frac{f_{-}^{2}(x)}{f_{+}^{2}(x-i \hbar \beta)} w(x)
$$


that is meromorphic, even and $2 \omega$-periodic in $x$. Moreover, it has no poles for real $x$ and is positive for $x \in(0,2 \omega)$, and it satisfies

$$
\lim _{\beta \rightarrow 0} w(\beta ; x)=C \exp \left(2 \frac{g}{\hbar} \ln s(x)\right), \quad x \in(0,2 \omega) .
$$

Here, the positive constant $C$ is irrelevant for what follows, and the logarithm is chosen real. (Note that (2.63) entails positivity of $s(x)$ on $(0,2 \omega)$.) Setting

$$
\Delta(\beta ; x) \equiv C^{\prime} \prod_{j<k} w\left(\beta ; x_{j}-x_{k}\right), \quad C^{\prime}>0
$$

and using (4.37), evenness of $w$ and (4.44), we obtain transformed A $\Delta \mathrm{OS}_{\mathrm{s}}$

$$
S_{l}^{t} \equiv \Delta^{-1 / 2} S_{l} \Delta^{1 / 2}=\sum_{\substack{I \subset\{1, \ldots, N\} \\|I|=l}} \prod_{\substack{j \in I \\ k \notin I}} f_{-}^{2}\left(x_{j}-x_{k}\right) \exp \left(\beta \hat{p}_{I}\right) .
$$

Next, we replace $S_{l}$ by $S_{l}^{t}$ in (4.40), obtaining an $\mathrm{A} \Delta \mathrm{O} A_{k}^{t}(\beta)$. In view of (4.45), the formal power series expansion of this $\mathrm{A} \Delta \mathrm{O}$ has as its lowest coefficient the PDO

$$
A_{k, n_{k}}^{t}=\Pi(x)^{-1} A_{k, n_{k}} \Pi(x)
$$

with

$$
\Pi(x) \equiv \prod_{j<k}\left|s\left(x_{j}-x_{k}\right)\right|^{g / \hbar} .
$$

To prove $n_{k}=k$, it therefore suffices to show that $A_{k, l}^{t}$ vanishes for $l<k$.

The crux is now, that we need only show this for the classical version $A_{k}^{t}(x, p)$. Indeed, in $S_{k}^{t}(4.46)$ all partials occur to the right of $\hbar$-independent coefficients, so the PDO $A_{k, l}^{t}$ is obtained by substituting (4.1) in the normally ordered expansion coefficient $A_{k, l}^{t}(x, p)$. Here, normal ordering denotes the procedure of putting $x$-dependent coefficients to the left of monomials in $p_{1}, \ldots, p_{N}$ (classical case) or $\hat{p}_{1}, \ldots, \hat{p}_{N}$ (quantum case); we shall symbolize normal ordering by double dots in the sequel. In particular, we can now rewrite (4.47) as

$$
A_{k, n_{k}}=\Pi(x): A_{k, n_{k}}^{t}(x, \hat{p}): \Pi(x)^{-1} .
$$

We proceed by proving that $A_{k, l}^{t}(x, p)$ vanishes for $l<k$. To this end we first notice that the function $c_{l}(\lambda, i \beta g) S_{l}^{t}(x, p)$ is the $l$ th symmetric function of the matrix

$$
\tilde{L}_{j k} \equiv \tilde{d}_{j} D_{j k}
$$


with $D$ given by (3.47), and

$$
\tilde{d}_{j} \equiv \exp \left(\beta p_{j}\right) \prod_{l \neq j} f_{-}^{2}\left(x_{j}-x_{l}\right) .
$$

(To verify this, one need only repeat the calculation leading to (3.48), making the pertinent changes in (3.37); note that $f_{-}^{2}(x) f_{-}^{2}(-x)=f^{2}(x)$.)

Next, we observe that

$$
\tilde{L}=1_{N}+\beta\left(L_{\mathrm{nr}}+E\right)+O\left(\beta^{2}\right), \quad \beta \rightarrow 0,
$$

where the extra matrix is given by

$$
E \equiv \operatorname{diag}\left(z_{1}(x), \ldots, z_{N}(x)\right)
$$

with

$$
z_{j}(x) \equiv-i g \sum_{l \neq j} \frac{s^{\prime}\left(x_{j}-x_{l}\right)}{s\left(x_{j}-x_{l}\right)}, \quad j=1, \ldots, N .
$$

Now this entails

$$
\lim _{\beta \rightarrow 0} \beta^{-k} A_{k}^{t}(\beta)=S_{k}\left(L_{\mathrm{nr}}+E\right) .
$$

From this we deduce not only that $A_{k, l}^{t}(x, p)$ vanishes for $l<k$ (as desired), but also-returning to the quantum level-that

$$
A_{k, k}=\Pi(x): S_{k}\left(L_{\mathrm{nr}}(x, \hat{p})+E(x)\right): \Pi(x)^{-1} .
$$

The upshot is that we have now derived integrability of the nonrelativistic type IV quantum system from that of its relativistic version. Moreover, we have obtained rather explicit formulas for the commuting PDOs $A_{k, k}$. To exploit the formula (4.50), it is important to observe (recall (4.48) and (4.49))

$$
\begin{gathered}
\Pi(x) \hat{p}_{j} \Pi(x)^{-1}=\hat{p}_{j}-z_{j}(x), \\
\hat{p}_{j}\left(z_{i}\right)=-g \hbar\left(\mathcal{P}\left(x_{j}-x_{i}\right)+\frac{\eta}{\omega}\right) \equiv-\tilde{h}\left(x_{j}-x_{i}\right), \quad j \neq i .
\end{gathered}
$$

Let us call the latter formula a contraction. Expanding (4.50) and using (4.51), we may move all $\hat{p}_{j}$ 's to the right, picking up contractions along the way. Then we wind up with an expression that can be written

$$
A_{k, k}=S_{k}\left(L_{\mathrm{nr}}(x, \hat{p})\right)+R_{k}(x, \hat{p})
$$

where $R_{k}(x, p)$ is normally ordered and consists of all terms involving at least one contraction. As such, $R_{k}(x, p)$ has degree $<k-1$ in $p$. From 
this one easily deduces that $A_{k, k}(x, \hat{p})$ has the form (4.25), (4.26). (For $k=1$ and $k=2$ we reobtain (4.42) and (4.43), respectively, as should be the case, of course.) Therefore, we are now in the position to invoke the uniqueness result of Ref. 49 detailed in the paragraph containing (4.27): The commutative algebra generated by $A_{k, k}, k=1, \ldots, N$, coincides with the algebra generated by $J_{1}(h), \ldots, J_{N}(h)$, with

$$
h(x) \equiv-g(g-\hbar) \mathcal{P}(x) \text {. }
$$

It is not hard to see that $A_{k, k}$ is actually a linear combination of $J_{1}$, $\ldots, J_{k}$ : Any monomial in $A_{k, k}(x, p)$ involves a given $p_{j}$ only once, so no products of the $J_{l}$ can occur. Notice also that the contractions are responsible for changing $g^{2}$ to $g(g-\hbar)$ in (4.54). In fact, we conjecture that one has

$$
\Pi(x) \sum_{|I|=l}: \prod_{i \in I}\left(\hat{p}_{i}+z_{i}(x)\right): \Pi(x)^{-1}=J_{l}(\tilde{h}), \quad l=1, \ldots, N,
$$

with $\tilde{h}$ given by (4.52). (We have checked this for $l \leq 4$.) Clearly, (4.55) would be useful to render the formula (4.53) for $A_{k, k}$ even more explicit.

Of course, the above holds true for the type I-III systems, too. If one could prove (4.55), it would easily follow from the IV $\rightarrow \mathrm{V}$ and II $\rightarrow$ VI transitions that one has

$$
A_{T, k, k}=S_{k}\left(L_{\mathrm{nr}}(x, \hat{p})\right)
$$

in the Toda case. (Here, $A_{T, k, k}$ denotes the $k$ th expansion coefficient of the $\mathrm{A} \Delta \mathrm{O} A_{T, k}(\beta)$ that is defined via (4.40) with $c_{l} S_{l}$ replaced by $S_{l}(4.38)$ for type VI and by $\Sigma_{l}(3.52)$ (with $S_{l}$ given by (4.38)) for type V.) In particular, this would entail that the quantized symmetric functions of the Lax matrices (2.57) and (2.59) commute.

Independent of the validity of (4.56), it can be shown that $n_{k}=k$ in the Toda case [5]; the reasoning presented in Ref. 5 applies to type I-III as well, but leaves open the type IV case. (The statements in Ref. 5 concerning the relation of $A_{k, k}$ and quantized symmetric functions should be ignored, though; this is because these rely on the unproven assertions in Refs. 1 and 2 discussed above.)

\section{Action-Angle Transforms}

\subsection{Introductory Examples}

At the end of Section 2.1 we have introduced action-angle maps, a notion associated with an arbitrary Liouville integrable system

$$
\mathcal{S}=\left\langle\Omega, \omega, I_{1}, \ldots, I_{N}\right\rangle
$$


As we have seen in Sections 2 and 3, the Calogero-Moser and Toda systems and their relativistic generalizations are Liouville integrable, so one is led to the problem of constructing explicit action-angle maps for these systems. Now the Liouville-Arnol'd theorem is of little help in that enterprise, since it is merely concerned with existence and general structure under some quite subtle assumptions, whose direct verification is often intractable. Provided the joint orbits (2.14) contained in $\Omega_{i}$ are compact (so that $k_{i}=N$ in (2.16)), there exist integral representations for the action and angle variables. (These are actually the reason for the term integrable system, historically speaking.) Even so, one would really like to express these integrals in terms of known functions, or obtain at least more information on the range of variation of the actions, the functional dependence of $I_{1}, \ldots, I_{N}$ on the actions, etc. More generally, the decomposition into invariant submanifolds $\Omega_{1}, \Omega_{2}, \ldots$ should be made explicit.

In order to provide more perspective for this circle of problems, we shall consider some quite elementary examples. Our starting point is the Hamiltonian

$$
H(x, p)=\frac{p^{2}}{2}+V(x), \quad V \in C^{\infty}(\mathbb{R})
$$

defined on $\Omega=\mathbb{R}^{2}$ equipped with its standard form $\omega=d x \wedge d p$. Then we have $d H\left(x_{0}, p_{0}\right) \neq 0$ unless both $p_{0}=0$ and $V^{\prime}\left(x_{0}\right)=0$; such points yield equilibrium solutions $(x(t), p(t))=\left(x_{0}, p_{0}\right), \forall t \in \mathbb{R}$. Since $d H \neq 0$ on an open dense set, $H$ is integrable.

The $H$ flow is not complete without further restrictions on $V$, however. For instance, taking $V=-x^{4} / 2$, one gets a solution $\left(1 /(1-t), 1 /(1-t)^{2}\right)$ to Hamilton's equations (2.1) that is defined only for $t<1$. To ensure completeness (and hence Liouville integrability), let us henceforth assume that $V$ is bounded below. (By energy conservation this entails an upper bound on $|p|$; since one has $\dot{x}=p$, this prevents escape to infinity in finite time.)

Of course, the simplest external field with this property is the constant field

$$
V(x)=V_{0}
$$

Discarding the line of equilibria $\{p=0\}$, one is left with two open, connected and invariant submanifolds $\Omega_{1}$ and $\Omega_{2}$ on which $p>0$ and $p<0$, respectively. These are of the form $\mathbb{R} \times A_{i}$ with $A_{i}$ open and connected, and the flow $(x(t), p(t))=\left(x_{0}+p_{0} t, p_{0}\right)$ is obviously linear in time. Hence one can take $\widehat{\Omega}_{i}=\Omega_{i}$ and $\Phi_{i}$ equal to the identity, $i=1,2$.

Next, we consider the harmonic field

$$
V(x)=\frac{x^{2}}{2} .
$$


In this case one clearly obtains the flow

$$
e^{t H}\left(x_{0}, p_{0}\right)=\left(x_{0} \cos t+p_{0} \sin t, p_{0} \cos t-x_{0} \sin t\right) .
$$

This flow is nonlinear in $t$, but away from the equilibrium $(0,0)$, it can be linearized by a symplectic map

$$
\Phi:\left\langle\Omega_{1}, d x \wedge d p\right\rangle \rightarrow\langle\widehat{\Omega}, d \hat{x} \wedge d \hat{p}\rangle, \quad(x, p) \mapsto(\hat{x}, \hat{p}),
$$

where

$$
\begin{aligned}
\Omega_{1} & \equiv \Omega \backslash\{(0,0)\} \\
\widehat{\Omega} & \equiv \mathbb{T}^{1} \times(0, \infty) .
\end{aligned}
$$

As announced below (2.17), the torus $\mathbb{T}^{1}$ is viewed as $\mathbb{R} / 2 \pi \mathbb{Z}$ and coordinatized by $\hat{x} \in(-\pi, \pi]$; explicitly, $\Phi$ reads

$$
\hat{x} \equiv \arctan (x / p), \quad \hat{p} \equiv\left(p^{2}+x^{2}\right) / 2=H(x, p),
$$

so that the inverse $\mathcal{E}$ is given by

$$
x=(2 \hat{p})^{1 / 2} \sin \hat{x}, \quad p=(2 \hat{p})^{1 / 2} \cos \hat{x},
$$

and one has

$$
\exp (t \widehat{H})\left(\hat{x}_{0}, \hat{p}_{0}\right)=\left(\hat{x}_{0}+t, \hat{p}_{0}\right) .
$$

In this example all orbits are periodic, but in general a Hamiltonian of the form (5.2) has both periodic and nonperiodic orbits. Indeed, taking as a third example

$$
V(x)=-\exp \left(-x^{2}\right)
$$

the level set $H(x, p)=E$ yields an equilibrium for $E=-1$, a periodic orbit for $E \in(-1,0)$, and two nonperiodic orbits for $E \geq 0$. Discarding the equilibrium at $(0,0)$ and the level set $E=0$ separating bound and unbound orbits (separatrix), one obtains three open, connected and invariant submanifolds $\Omega_{1}, \Omega_{2}, \Omega_{3}$, corresponding to $E \in(-1,0)$, and to $E>0$ with $p>0$ and $p<0$, respectively.

The action variable $\hat{p}(x, p)$ on $\Omega_{1}$ is now determined (uniquely up to a constant) as the function $f(H(x, p))$ that is such that all of its orbits have primitive period $2 \pi$. It can be seen that the function

$$
\begin{gathered}
f(E)=\frac{1}{2 \pi} A(E)=\frac{2}{\pi} \int_{0}^{V^{-1}(E)} p(x, E) d x, \quad E \in(-1,0), \\
p(x, E) \equiv\left(\frac{E-V(x)}{2}\right)^{1 / 2}, \quad V(x) \leq E
\end{gathered}
$$


has this property. Here, $A(E)$ denotes the phase space area enclosed by the level curve $H(x, p)=E$. Note this yields $\hat{p}=H$ for the special case (5.3), in agreement with (5.6). For (5.8) or any other potential with the same shape (such as $-1 / \operatorname{ch}^{2} x$ ), the oscillation period is nonconstant on $\Omega_{1}$, so the transformed Hamiltonian must be a nonlinear function of $\hat{p}$. (In this regard the above harmonic oscillator example is highly nongeneric.)

There is also an integral representation for the canonically conjugate angle variable $\hat{x} \in(-\pi, \pi$ ] (cf. Ref. 9, p. 281); this variable is uniquely determined up to addition $(\bmod 2 \pi)$ of an arbitrary function of $\hat{p}$. Since we have $H=f^{-1} \circ \hat{p}$, the $H$ flow increases the angle by $2 \pi$ after a time $2 \pi \partial f / \partial E \equiv T(E)$. Using (5.9) and (5.10) to calculate $T(E)$, one now verifies that this yields the correct oscillation period.

The integral trajectories in $\Omega_{2}$ and $\Omega_{3}$ are scattering orbits, and so one has far more freedom in the choice of action coordinate. Indeed, one can take $\hat{p}=f(H)$ for any function $f \in C^{\infty}((0, \infty))$ with positive or negative derivative on $(0, \infty)$, and obtain a canonically conjugate angle variable $\hat{x}(x, p)$ taking values in $\mathbb{R}$. There is, however, a special choice that is singled out by a physical interpretation: one can choose $\hat{p}(x, p)$ equal to the limit of $p(t)$ for $t \rightarrow \infty$, so that $\hat{p}=(2 H)^{1 / 2}$ on $\Omega_{2}$ and $\hat{p}=-(2 H)^{1 / 2}$ on $\Omega_{3}$. As canonically conjugate position one can choose the asymptotic position $x^{+}$ determined by

$$
(x(t), p(t)) \sim\left(x^{+}+t \hat{p}, \hat{p}\right), \quad t \rightarrow \infty .
$$

Alternatively, one can work with the $t \rightarrow-\infty$ asymptotics, yielding actionangle variables $\left(x^{-}, \hat{p}\right)$. Restricting attention to $\Omega_{2}$, canonicity of the maps

$$
U_{ \pm}: \mathbb{R} \times(0, \infty) \equiv \widehat{\Omega}_{2} \rightarrow \Omega_{2}, \quad\left(x^{ \pm}, \hat{p}\right) \mapsto(x, p),
$$

is then a consequence of scattering theory. Indeed, they are just the wave maps

$$
\begin{gathered}
U_{ \pm}=\lim _{t \rightarrow \pm \infty} \exp (-t H) \circ \exp (t \widehat{H}), \\
\widehat{H}\left(x^{ \pm}, \hat{p}\right) \equiv \frac{\hat{p}^{2}}{2}=U_{ \pm}^{*} H
\end{gathered}
$$

which are related via the scattering map

$$
S \equiv U_{+}^{-1} U_{-}: \widehat{\Omega}_{2} \rightarrow \widehat{\Omega}_{2}, \quad\left(x^{-}, \hat{p}\right) \mapsto\left(x^{+}, \hat{p}\right) .
$$

(Writing

$$
x^{+}=x^{-}+\delta(\hat{p})
$$

the scattering is encoded in the function $\delta(\hat{p})$ : it registers how much the particle has advanced as compared to a freely moving particle with velocity 
$\hat{p}$.) Summarizing, one can choose the action-angle map $\Phi_{2}$ equal to $U_{+}^{-1}$ or $U_{-}^{-1}$.

As a fourth example, let us take

$$
V(x)=x^{2} \exp \left(-x^{2}\right)
$$

This potential has a well around the origin and two maxima $V( \pm 1)=e^{-1}$. Thus the level set $E=0$ yields an equilibrium $(0,0)$, whereas the level set $E \in\left(0, e^{-1}\right)$ splits up into three orbits: Two scattering orbits (reflection at the bumps) and one periodic orbit (oscillation around the origin). For $E>e^{-1}$ the level set has two connected components, corresponding to scattering orbits (transmission from left and right). The separatrix level set $E=e^{-1}$ is connected, but not diffeomorphic to $\mathbb{R}$ or $\mathbb{T}^{1}$. Indeed, this set splits up into eight distinct orbits: Discarding the two equilibria at $( \pm 1,0)$, one is left with six connected components, each of which yields an orbit. (Draw the phase diagram to see this.) Deleting the origin and the separatrix from $\Omega=\mathbb{R}^{2}$, one obtains a set with five connected components, viz., one bound state submanifold $\Omega_{1}$, two reflection submanifolds $\Omega_{2}, \Omega_{3}$ and two transmission submanifolds $\Omega_{4}, \Omega_{5}$. On each of these the flow can be linearized by a canonical map $\Phi_{i}$, involving the 1-torus and the integral (5.9) on $\Omega_{1}$, and scattering theory objects on $\Omega_{2}, \ldots, \Omega_{5}$.

Turning now to the case $N>1$, we note that there is a trivial, yet instructive way of manufacturing Liouville integrable systems: One can take

$$
I_{j}(x, p) \equiv \frac{p_{j}^{2}}{2}+V_{j}\left(x_{j}\right), \quad j=1, \ldots, N, V_{j} \in C^{\infty}(\mathbb{R}), V_{j} \geq 0
$$

to get $N$ commuting Hamiltonians on $\Omega=\mathbb{R}^{2 N}$ with its standard form (2.3). The submanifolds $\Omega_{1}, \Omega_{2}, \ldots$ and action-angle maps $\Phi_{1}, \Phi_{2}, \ldots$ then have a product structure, and the complement of $\Omega_{1} \cup \Omega_{2} \cup \cdots$ will consist of a union of sets that are built up from equilibria and separatrices for each of the Hamiltonians $I_{1}, \ldots, I_{N}$. The dimension of the latter excluded sets can vary from $2 N-1$ (take, e.g., $\left(x_{1}, p_{1}\right)$ on a separatrix for $I_{1}$ ) to 0 (take $\left(x_{j}, p_{j}\right)$ to be an equilibrium for $\left.I_{j}, j=1, \ldots, N\right)$. Moreover, the integer $k_{i}$ in (2.16) takes all allowed values $0, \ldots, N$ when each of $I_{1}, \ldots, I_{N}$ has both bound and scattering orbits.

From these examples one already gleans that the $N>1$ situation can be quite complicated. An additional complication for $N>1$ cannot be easily illustrated, since it is absent for the rather artificial product situation just discussed: To obtain invariant submanifolds of the form (2.15), (2.16) one may have to discard additional separatrices whose location is a matter of choice (just as branch cuts ensuring one-valuedness can be chosen at will). In geometric parlance, this phenomenon amounts to the occurrence of a nontrivial fiber bundle structure (with the fiber equal to (2.16)). We shall encounter an explicit example of this situation toward the end of 
Section 5.3. Since the general theory should cover all possibilities at once, it is clear that one must make rather intricate technical assumptions in order to obtain invariant submanifolds that are symplectically diffeomorphic to manifolds of the form (2.15), (2.16). A detailed account can be found in Ref. 11; see also Ref. 52 for general information on the way in which the invariant tori can bifurcate and degenerate, and on various related matters.

\subsection{Wave Maps and Pure Soliton Systems}

In view of the general picture sketched in the previous subsection one should be prepared to encounter considerable complications in constructing explicit action-angle transforms for a given Liouville integrable system. There exists however a physically important class of integrable systems for which neither equilibria nor separatrices occur, so that one needs to consider only one invariant submanifold, namely, all of $\Omega$. These integrable systems are defined by the Hamiltonian $H(2.13)$ on the phase space $\Omega(2.23)$, with $V(x)$ a pair potential having the salient features of the type II potential $1 / \operatorname{sh}^{2} \nu x$. Specifically, $V(x)$ is a strictly monotone decreasing function on $(0, \infty)$ with a divergence for $x \downarrow 0$ preventing collisions, and rapid decay to 0 at $\infty$. Let us denote the class of such repulsive potentials by $\mathcal{R}$, and fix $V \in \mathcal{R}$. We proceed by describing how the inverses of the wave maps from scattering theory can now be used as action-angle maps, in much the same way as for the above example; cf. (5.11)-(5.12). (In the example, however, the potential is attractive, so that transmission occurs.)

First of all, any point in $\Omega$ yields an orbit with asymptotics

$$
(x(t), p(t)) \sim\left(x^{ \pm}+t p^{ \pm}, p^{ \pm}\right), \quad t \rightarrow \pm \infty,
$$

where

$$
p_{1}^{+}>\cdots>p_{N}^{+}, \quad p_{1}^{-}<\cdots<p_{N}^{-} .
$$

Introducing incoming and outgoing phase spaces

$$
\begin{aligned}
& \Omega^{+} \equiv\left\{\left(x^{+}, p^{+}\right) \in \mathbb{R}^{2 N} \mid p_{N}^{+}<\cdots<p_{1}^{+}\right\} \\
& \Omega^{-} \equiv\left\{\left(x^{-}, p^{-}\right) \in \mathbb{R}^{2 N} \mid p_{N}^{-}>\cdots>p_{1}^{-}\right\}
\end{aligned}
$$

equipped with their canonical forms

$$
\omega^{\delta} \equiv \sum_{j=1}^{N} d x_{j}^{\delta} \wedge d p_{j}^{\delta}, \quad \delta=+,-,
$$

and Hamiltonians

$$
H^{\delta}\left(x^{\delta}, p^{\delta}\right) \equiv \frac{1}{2} \sum_{j=1}^{N}\left(p_{j}^{\delta}\right)^{2}, \quad \delta=+,-,
$$


the wave maps are given by

$$
U_{\delta} \equiv \lim _{t \rightarrow \delta \infty} \exp (-t H) \circ \exp \left(t H^{\delta}\right), \quad \delta=+,-
$$

Observe that the composition makes sense for $t \rightarrow \delta \infty$ : the restrictions (5.13) ensure that the point $\left(x^{\delta}+t p^{\delta}, p^{\delta}\right)$ belongs to $\Omega(2.23)$ for $\delta t$ large enough. Since the Hamiltonian flows at the right-hand side of (5.16) are canonical, the wave maps are symplectic maps

$$
U_{\delta}: \Omega^{\delta} \rightarrow \Omega, \quad\left(x^{\delta}, p^{\delta}\right) \mapsto(x, p) .
$$

(To prove this rigorously is not easy, though.) The intertwining relations

$$
U_{\delta} \circ \exp \left(t H^{\delta}\right)=\exp (t H) \circ U_{\delta}, \quad \delta=+,-,
$$

show that $U_{\delta}^{-1}$ can indeed be viewed as a linearizing canonical transformation $\Phi_{\delta}: \Omega \rightarrow \Omega^{\delta}$; cf. also (2.11), (2.12).

Liouville integrability of $H$ is now easily established. Indeed, defining

$$
H_{k}^{\delta}\left(x^{\delta}, p^{\delta}\right) \equiv \frac{1}{k} \sum_{j=1}^{N}\left(p_{j}^{\delta}\right)^{k}, \quad k=1, \ldots, N,
$$

the Hamiltonians $H_{1}^{\delta}, \ldots, H_{N}^{\delta}$ are obviously in involution. Moreover, they are independent; their gradients are in fact linearly independent on all of $\Omega^{\delta}$, since the relevant determinant is a Vandermonde determinant that has no zeros on $\Omega^{\delta}$. Setting

$$
\check{H}_{k}^{\delta} \equiv H_{k}^{\delta} \circ U_{\delta}^{-1}, \quad \delta=+,-, k=1, \ldots, N,
$$

it follows that $\check{H}_{1}^{\delta}, \ldots, \check{H}_{N}^{\delta}$ are in involution, and that their gradients are linearly independent on all of $\Omega$. Since we have

$$
H_{2}^{\delta}=H^{\delta}, \check{H}_{2}^{\delta}=H, \quad \delta=+,-,
$$

in view of (5.15) and the intertwining relations (5.17), it follows that $H$ is integrable. Moreover, because the map $U_{\delta}$ is canonical, (5.18) entails that it intertwines the flows $\exp \left(t H_{k}^{\delta}\right)$ and $\exp \left(t \check{H}_{k}^{\delta}\right)$. Since the former flows are manifestly complete, the latter are, too. Hence, $H$ is Liouville integrable, as claimed.

Now for a general $V \in \mathcal{R}$ it is quite unlikely that the commutative subalgebras of $\mathcal{I}_{H}$ generated by $\check{H}_{1}^{+}, \ldots, \check{H}_{N}^{+}$and $\check{H}_{1}^{-}, \ldots, \check{H}_{N}^{-}$are equal, and even more unlikely that $\check{H}_{k}^{+}$and $\breve{H}_{k}^{-}$are equal for $k>2$. (By translation invariance one does have equality for $k=1$. Indeed, in that case one obtains the total momentum $\sum_{j=1}^{N} p_{j}$.) Assuming however that they are equal, it follows that

$$
\sum_{j=1}^{N} p_{j}^{+}(x, p)^{k}=\sum_{j=1}^{N} p_{j}^{-}(x, p)^{k}, \quad k=1, \ldots, N
$$


and so we deduce that the scattering map

$$
S=U_{+}^{-1} U_{-}: \Omega^{-} \rightarrow \Omega^{+}, \quad\left(x^{-}, p^{-}\right) \mapsto\left(x^{+}, p^{+}\right)
$$

conserves momenta. More specifically, from (5.13) we deduce (2.47).

Conversely, whenever $S$ conserves momenta, one has the equalities (5.19) and so the pullback Hamiltonians $\check{H}_{k}^{+}$and $\check{H}_{k}^{-}$are equal for $k=1, \ldots, N$. A priori, these Hamiltonians need not have a polynomial dependence on the momenta $p_{1}, \ldots, p_{N}$, however. But for the only potentials in $\mathcal{R}$ for which conservation of momenta is known to hold true, this turns out to be the case. Indeed, these potentials are the type II potentials $V(x)=1 / \operatorname{sh}^{2} \nu x$, whose long-time asymptotics has already been discussed in Section 2.2 ; cf. (2.42)-(2.48). In view of (2.46) and (2.33) one has

$$
\check{H}_{k}^{\delta}=H_{k}, \quad \delta=+,-, k=1, \ldots, N,
$$

and $H_{k}$ is indeed a polynomial in $p_{1}, \ldots, p_{N}$ with $x$-dependent coefficients; cf. (2.41).

It is not known whether any other $V \in \mathcal{R}$ exist for which $S$ conserves momenta, but this seems extremely unlikely. At any rate, it makes sense to single out the integrable systems (5.1) for which not only each initial state is a scattering state, but also the momenta are conserved under the scattering. We shall call such systems pure soliton systems. As we have seen in Sections 2 and 3, the systems of type I, II, and VI are pure soliton systems, not only at the nonrelativistic, but also at the relativistic level. In the next subsection we shall obtain detailed information on the type I and II wave and scattering maps, but for brevity we do not consider the type VI case. (A study of the latter case can be found in Ref. 37.)

\subsection{Systems of Type I, II, and III}

As explained in the previous subsection, one can choose one of the two inverse wave maps $U_{+}^{-1}, U_{-}^{-1}$ as an action-angle map for the systems of type $I$ and Ii. Indeed, the action-angle map $\Phi$ we are going to construct will turn out to be equal to $U_{+}^{-1}$ for the type I systems, but for type II it is slightly different, and the difference will be crucial. In all four cases the map is written

$$
\Phi: \Omega \rightarrow \widehat{\Omega}, \quad(x, p) \mapsto(\hat{x}, \hat{p}),
$$

with $\Omega$ the type I and II phase space (2.23), and $\widehat{\Omega}$ the outgoing phase space $\Omega^{+}$(5.14). Hence, $\Omega$ and $\widehat{\Omega}$ are related by

$$
\widehat{\Omega}=I(\Omega), \quad I(x, y) \equiv(y, x), \quad x, y \in \mathbb{R}^{N} .
$$

For the type III systems we should consider three distinct phase spaces; cf. (2.24)-(2.30). As it happens, it suffices to delete a codimension-2 variety containing partial equilibria from each of these to obtain an invariant 
submanifold on which an action-angle map can be defined. Thus, no separatrices occur in this case, too. (Recall that a manifold remains connected after discarding a subset whose codimension is greater than one.)

The key tool in the construction of the action-angle map for all of the systems of type I-III is a commutation relation of the Lax matrix defined in Sections 2.2 and 3.1 with a diagonal matrix $A(x)$ given by

$$
A(x)=\operatorname{diag}\left(d\left(x_{1}\right), \ldots, d\left(x_{N}\right)\right), \quad d(y) \equiv\left\{\begin{array}{l}
y, \\
\exp (\mu y), \\
\exp (i \mu y) .
\end{array}\right.
$$

Specializing first to the type I and II cases, the symmetric functions $\check{D}_{k}(x)$ of $A(x)$ evidently give rise to an integrable system on $\Omega$, so the Hamiltonians

$$
D_{k} \equiv \check{D}_{k} \circ \mathcal{E}, \quad \mathcal{E} \equiv \Phi^{-1}
$$

yield an integrable system on the action-angle phase space $\widehat{\Omega}$. Now this would not be of much interest by itself; in fact, this observation applies to any diagonal matrix of the form (5.22) with $d^{\prime}(x)>0$, say. The crux is, however, that the integrable systems thus obtained are of physical interest: they are type I or II systems!

Specifically, denoting the dual systems just defined by a caret, they are given by

$$
\begin{aligned}
& \hat{\mathrm{I}}_{\mathrm{nr}} \simeq \mathrm{I}_{\mathrm{nr}}, \quad \hat{\mathrm{I}}_{\mathrm{rel}} \simeq \mathrm{II}_{\mathrm{nr}}, \\
& \widehat{\mathrm{II}}_{\mathrm{nr}} \simeq \mathrm{I}_{\mathrm{rel}}, \quad \widehat{\mathrm{I}}_{\mathrm{rel}} \simeq \mathrm{II}_{\mathrm{rel}} .
\end{aligned}
$$

Moreover, the inverse $\mathcal{E}$ of $\Phi$ serves as an action-angle map for these systems, as can already be seen from (5.23).

For the type III case the function $d(y)(5.22)$ is not real-valued, but now one can for example consider the symmetric functions of the matrix $A(x)+A(x)^{*}$. As it turns out, the dual systems arising from the latter Hamiltonians are once more pure soliton systems (in a slightly more general sense than described in the previous subsection), and the action-angle maps are intimately related to the wave maps for these pure soliton systems. We shall return to the dual type III systems later on.

We continue by supplying the details of the construction of $\Phi$ for the relativistic type II systems. The above-mentioned commutation relatior reads

$$
\frac{1}{2} \operatorname{coth}(z)[A, L]=e \otimes e-\frac{1}{2}(A L+L A)
$$

where we take

$$
z \equiv \frac{i \beta \mu g}{2} \in i(0, \pi)
$$


Also, $e$ is the vector-valued function given by (3.36), $L$ is the Lax matrix (3.35), and $A$ the diagonal matrix (5.22). (Note that (5.25) is invariant under taking $z, A, L \rightarrow-z, L, A$; this symmetry property will eventually lead to the self-duality of the $\mathrm{II}_{\mathrm{rel}}$ system.) Since $L$ is self-adjoint, there exists a unitary $U$ such that

$$
\hat{L} \equiv U^{*} L U=\operatorname{diag}\left(\lambda_{1}, \ldots, \lambda_{N}\right), \quad \lambda_{i} \in \mathbb{R} .
$$

Transforming (5.25) with $U$ and setting

$$
\hat{A} \equiv U^{*} A U, \quad \hat{e} \equiv U^{*} e,
$$

one readily obtains

$$
\frac{1}{2} \hat{A}_{j k}\left[\operatorname{coth}(z)\left(\lambda_{k}-\lambda_{j}\right)+\lambda_{k}+\lambda_{j}\right]=\hat{e}_{j} \overline{\hat{e}}_{k}
$$

Now $A$ has positive spectrum, and since $U$ is unitary, it follows that $\hat{A}_{j j}>$ 0 . Moreover, we have

$$
\prod_{j=1}^{N} \lambda_{j}=|\hat{L}|=|L|=\exp \left(\beta\left(p_{1}+\cdots+p_{N}\right)\right)>0
$$

so that $\lambda_{j} \neq 0, j=1, \ldots, N$. Taking $j=k$ in (5.27) we deduce $\hat{e}_{j} \neq 0$ and $\lambda_{j}>0$. Consequently, we may define a vector $\hat{p} \in \mathbb{R}^{N}$ by setting

$$
\lambda_{j} \equiv \exp \left(\beta \hat{p}_{j}\right), \quad j=1, \ldots, N .
$$

Next, we rewrite (5.27) as

$$
\hat{A}_{j k}=\hat{e}_{j} \overline{\hat{e}}_{k} \exp \left(-\frac{\beta\left(\hat{p}_{j}+\hat{p}_{k}\right)}{2}\right) \frac{\operatorname{sh} z}{\operatorname{sh}\left(\beta\left(\hat{p}_{k}-\hat{p}_{j}\right) / 2+z\right)} .
$$

Recalling (3.33), (3.34), and (3.23) we deduce

$$
|\hat{A}|=\prod_{j}\left(\left|\hat{e}_{j}\right|^{2} \exp \left(-\beta \hat{p}_{j}\right)\right) \prod_{j<k}\left(\frac{\operatorname{sh}^{2}\left(\beta\left(\hat{p}_{j}-\hat{p}_{k}\right) / 2\right)}{\operatorname{sh}^{2}\left(\beta\left(\hat{p}_{j}-\hat{p}_{k}\right) / 2\right)+\sin ^{2}(\beta \mu g / 2)}\right)
$$

Now we have $|\hat{A}|=|A|>0$ and $\sin (\beta \mu g / 2)>0$ (cf. (5.26)), so that

$$
\hat{p}_{j} \neq \hat{p}_{k}, \quad 1 \leq j<k \leq N .
$$

The upshot is, that $L$ has positive and nondegenerate spectrum. The gauge ambiguity in the diagonalizing unitary is therefore given by the product of a permutation matrix and a diagonal phase matrix. To render $U$ unique, we get rid of this gauge freedom: We require

$$
\hat{L}=\operatorname{diag}\left(\exp \left(\beta \hat{p}_{1}\right), \ldots, \exp \left(\beta \hat{p}_{N}\right)\right), \quad \hat{p}_{N}<\cdots<\hat{p}_{1},
$$


to fix the permutation matrix, and

$$
\hat{e}_{j}>0, \quad j=1, \ldots, N
$$

to fix the phase matrix. (Recall $\hat{e} \equiv U^{*} e$; also, recall we have already shown $\hat{e}_{j} \neq 0$, so (5.30) makes sense.)

Since $U$ is now uniquely determined, the vector $\hat{e}$ is uniquely determined, too. Therefore, we can now introduce a vector $\hat{x} \in \mathbb{R}^{N}$ by parametrizing $\hat{e}_{j}$ as

$$
\hat{e}_{j}=\exp \left(\frac{\beta \hat{p}_{j}+\mu \hat{x}_{j}}{2}\right) \prod_{l \neq j}\left(1+\frac{\sin ^{2}(\beta \mu g / 2)}{\operatorname{sh}^{2}\left(\beta\left(\hat{p}_{j}-\hat{p}_{l}\right) / 2\right)}\right)^{1 / 4} .
$$

This ensures that the map $\Phi(5.20)$ satisfies

$$
\hat{e}(\hat{x}, \hat{p})=e(\mu, \beta, g ; \hat{p}, \hat{x})
$$

where $e(\beta, \mu, g ; x, p)$ is given by (3.36), (3.23); recalling (5.28), this entails

$$
\hat{A}(\hat{x}, \hat{p})=L(\mu, \beta, g ; \hat{p}, \hat{x})^{t}
$$

where $L(\beta, \mu, g ; x, p)$ is given by (3.35).

The relation (5.33) amounts to the self-duality announced previously (cf. (5.24)). Indeed, it entails that the symmetric functions of $\hat{A}(\hat{x}, \hat{p})$ equal the previous Hamiltonians $S_{k}$, with $\beta$ and $\mu$ interchanged and $\hat{p}_{j}$ and $\hat{x}_{j}$ playing the role of positions and momenta, respectively.

To show that $\Phi(\beta, \mu, g ; x, p)$ is actually a bijection onto $\widehat{\Omega}(5.21)$, it suffices to construct a map $\mathcal{E}(\beta, \mu, g ; \hat{x}, \hat{p}): \widehat{\Omega} \rightarrow \Omega$ that satisfies

$$
\mathcal{E} \circ \Phi=\mathrm{id}(\Omega), \quad \Phi \circ \mathcal{E}=\mathrm{id}(\widehat{\Omega}) .
$$

In view of the self-duality relations (5.32), (5.33) this is quite straightforward: One need only run the construction backwards to obtain a map $\mathcal{E}$ with these two properties. Then it follows that $\mathcal{E}$ is the inverse of the bijection $\Phi$ and, in addition, one infers

$$
\mathcal{E}(\beta, \mu, g ; \hat{x}, \hat{p})=I \circ \Phi(\mu, \beta, g ; \hat{p}, \hat{x})
$$

where $I$ is the flip map (5.21). (The transpose in (5.33) can be traded for a sign flip of $g$, and it is not hard to see that $\Phi$ is even in $g$.)

A complete proof that the bijection $\Phi$ is in fact a canonical diffeomorphism involves more work. A crucial ingredient of the proof that can be found in Ref. 53 is the scattering theory associated to the $S_{1}$ flow, which was already sketched in Section 3.1. We shall return to the wave and scattering maps shortly, but it is convenient to obtain first a crucial finite-time result, which follows rather easily when one takes for granted that $\Phi$ is a symplectic map. 
We begin by observing that due to its spectral properties $L$ admits a logarithm. Specifically, we have

$$
\ln L=U \operatorname{diag}\left(\beta \hat{p}_{1}, \ldots, \beta \hat{p}_{N}\right) U^{*} ;
$$

cf. (5.29). For $h \in C^{\infty}(\mathbb{R})$ we can then define

$$
h\left(\beta^{-1} \ln L\right) \equiv U \operatorname{diag}\left(h\left(\hat{p}_{1}\right), \ldots, h\left(\hat{p}_{N}\right)\right) U^{*} .
$$

We now study Hamiltonians of the form

$$
H_{h} \equiv \operatorname{Tr} h\left(\beta^{-1} \ln L\right), \quad h \in C^{\infty}(\mathbb{R}) .
$$

These include the Hamiltonians $S_{ \pm 1}, H$ and $P$ from Section 3.1, since we have

$$
h(y)=\left\{\begin{array}{lll}
\exp ( \pm \beta y) & S_{ \pm 1} \\
\beta^{-2} \operatorname{ch} \beta y & \Rightarrow & H \\
\beta^{-1} \operatorname{sh} \beta y & P
\end{array}\right\}=H_{h} .
$$

Similarly, all power traces of $L$ are included (take $h(y)=\exp (k \beta y) / k$, $k=1,2, \ldots)$.

Let $t \in \mathbb{R}$ and $Q \equiv(x, p) \in \Omega$, and define the matrix

$$
A_{h}(t, Q) \equiv A(Q) \exp \left(t \mu h^{\prime}\left(\beta^{-1} \ln L(Q)\right)\right) .
$$

For $t=0$ this matrix reads $\operatorname{diag}\left(\exp \left(\mu x_{1}\right), \ldots, \exp \left(\mu x_{N}\right)\right)$, so it has manifestly positive and simple spectrum. We claim that it actually has positive and simple spectrum for all $t \in \mathbb{R}$. Furthermore, we claim that the $H_{h}$ flow is complete and that the configuration space projection of the integral curve $\exp \left(t H_{h}\right)(Q)$ is given by

$$
x_{j}(t)=\mu^{-1} \ln \alpha_{j}(t), \quad j=1, \ldots, N, 0<\alpha_{N}(t)<\cdots<\alpha_{1}(t),
$$

where $\alpha_{1}, \ldots, \alpha_{N}$ are the ordered eigenvalues of $A_{h}$.

Exploiting the above map $\Phi$ and its canonicity property, the proof of these claims is quite short. Indeed, setting

$$
\widehat{Q} \equiv \Phi(Q)=(\hat{x}, \hat{p}), \quad \widehat{H}_{h} \equiv H_{h} \circ \Phi^{-1},
$$

we have

$$
\widehat{H}_{h}(\widehat{Q})=\operatorname{Tr} U \operatorname{diag}\left(h\left(\hat{p}_{1}\right), \ldots, h\left(\hat{p}_{N}\right)\right) U^{*}=\sum_{j=1}^{N} h\left(\hat{p}_{j}\right) .
$$

Therefore, the $\widehat{H}_{h}$ flow on $\widehat{\Omega}$ reads

$$
\exp \left(t \widehat{H}_{h}\right)(\hat{x}, \hat{p})=\left(\hat{x}_{1}+t h^{\prime}\left(\hat{p}_{1}\right), \ldots, \hat{x}_{N}+t h^{\prime}\left(\hat{p}_{N}\right), \hat{p}\right)
$$


so it is manifestly complete. Since $\Phi$ is canonical, it follows from (5.37) that

$$
\exp \left(t H_{h}\right)=\mathcal{E} \circ \exp \left(t \widehat{H}_{h}\right) \circ \Phi .
$$

Hence the $H_{h}$ flow is complete, too, as claimed. Finally, denoting similarity by $\sim$, we obtain

$$
\begin{aligned}
A_{h}(t, Q) & \sim \hat{A}(\widehat{Q}) \exp \left(t \mu h^{\prime}\left(\beta^{-1} \ln \hat{L}(\widehat{Q})\right)\right) \\
& =\hat{A}(\hat{x}, \hat{p}) \operatorname{diag}\left(\exp \left(t \mu h^{\prime}\left(\hat{p}_{1}\right)\right), \ldots, \exp \left(t \mu h^{\prime}\left(\hat{p}_{N}\right)\right)\right) \\
& \sim \hat{A}\left(\hat{x}_{1}+t h^{\prime}\left(\hat{p}_{1}\right), \ldots, \hat{x}_{N}+t h^{\prime}\left(\hat{p}_{N}\right), \hat{p}\right) \\
& =\hat{A}\left(\exp \left(t \widehat{H}_{h}\right)(\widehat{Q})\right) \\
& \sim A\left(\exp \left(t H_{h}\right)(Q)\right)
\end{aligned}
$$

where we used $\hat{A}=U^{*} A U$ and (5.34) in the first and second steps, (5.28) and (5.31) in the third, (5.38) in the fourth, and (5.39) in the last step. The remaining claims are now evident from (5.40).

The results just proved yield a quite explicit description of the position part of the integral curve, which is especially useful to study its long-time asymptotics. Indeed, (5.40) entails that we can express this asymptotics in terms of the spectral asymptotics for $t \rightarrow \pm \infty$ of the matrix

$$
\hat{A}(\hat{x}, \hat{p}) \operatorname{diag}\left(\exp \left(t d_{1}\right), \ldots, \exp \left(t d_{N}\right)\right), \quad d_{j} \equiv \mu h^{\prime}\left(\hat{p}_{j}\right) .
$$

Let us now assume that $h^{\prime \prime}(y)>0$, so that $h^{\prime}(y)$ is strictly increasing. (This holds true for $S_{1}$ and $H$, for instance; cf. (5.36).) Since $\hat{A}$ is a positive matrix, we should determine the spectral asymptotics of matrices of the form

$$
E(t)=M e^{t D}, \quad M>0, D=\operatorname{diag}\left(d_{1}, \ldots, d_{N}\right), d_{N}<\cdots<d_{1} .
$$

For $t \rightarrow \infty(t \rightarrow-\infty)$, this can be expressed in terms of upper (lower) corner principal minors of $M$. Specifically, let us set

$$
\begin{gathered}
m_{1}^{+} \equiv M(1), \quad m_{2}^{+} \equiv \frac{M(1,2)}{M(1)}, \quad m_{3}^{+} \equiv \frac{M(1,2,3)}{M(1,2)}, \ldots, \\
m_{1}^{-} \equiv M(N), \quad m_{2}^{-} \equiv \frac{M(N-1, N)}{M(N)}, \ldots,
\end{gathered}
$$

so that $m_{1}^{+}=M_{11}, m_{2}^{+}=\left(M_{11} M_{22}-M_{12} M_{21}\right) / M_{11}$, etc.. Then it is proved in Appendix A of Ref. 53 that the (ordered) eigenvalues $\alpha_{1}(t), \ldots, \alpha_{N}(t)$ of $E(t)$ satisfy

$$
\exp \left(-t d_{j}\right) \alpha_{N-j+1}(t)-m_{N-j+1}^{ \pm}=O(\exp (\mp t R)), \quad t \rightarrow \pm \infty,
$$


where

$$
R \equiv \min \left(d_{1}-d_{2}, \ldots, d_{N-1}-d_{N}\right) .
$$

(Notice that these formulas are trivially true when $M$ is diagonal.)

To apply this to the concrete matrix (5.41), we need the relevant principal minors of $\hat{A}$. But these are easily calculated explicitly by using (5.33) and recalling (3.37). Proceeding in this way we find

$$
m_{j-j+1}^{ \pm}(\hat{A}(\hat{x}, \hat{p}))=\exp \left(\mu \hat{x}_{j} \mp \frac{\mu}{2} \Delta_{j}(\hat{p})\right)
$$

where

$$
\begin{aligned}
& \Delta_{j}(p)=\left(\sum_{k<j}-\sum_{k>j}\right) \delta\left(p_{j}-p_{k}\right), \\
& \delta(p)=\frac{1}{\mu} \ln \left(1+\frac{\sin ^{2}(\beta \mu g / 2)}{\operatorname{sh}^{2}(\beta p / 2)}\right) .
\end{aligned}
$$

Putting the pieces together, we obtain

$$
x_{N-j+1}(t)=\hat{x}_{j} \mp \frac{1}{2} \Delta_{j}(\hat{p})+t h^{\prime}\left(\hat{p}_{j}\right)+O(\exp (\mp t R)), \quad t \rightarrow \pm \infty
$$

and using isospectrality of $L\left(\exp \left(t H_{h}\right)(Q)\right)$ we deduce

$$
p_{j-j+1}(t)=\hat{p}_{j}+O(\exp (\mp t R)), \quad t \rightarrow \pm \infty .
$$

Now for the special case $h(y)=\exp (\beta y)$ we have $H_{h}=S_{1}$, so (5.42)(5.44) render the asymptotics $(3.22),(2.43)$ and (2.48) explicit: One has

$$
\begin{array}{rlrl}
x_{j}^{+} & =\hat{x}_{j}-\frac{1}{2} \Delta_{j}(\hat{p}), p_{j}^{+}=\hat{p}_{j}, & & j=1, \ldots, N, \\
x_{N-j+1}^{-} & =\hat{x}_{j}+\frac{1}{2} \Delta_{j}(\hat{p}), \quad p_{N-j+1}^{-}=\hat{p}_{j}, & j=1, \ldots, N,
\end{array}
$$

so that the scattering map reads

$$
x_{j}^{+}=x_{N-j+1}^{-}+\Delta_{N-j+1}\left(p^{-}\right), p_{j}^{+}=p_{N-j+1}^{-}, \quad j=1, \ldots, N .
$$

In particular, $\delta(p)(5.43)$ is the shift incurred in the two-particle interaction (as compared to a billiard ball collision). However, the results just obtained are far more general: They entail that the wave and scattering maps are shared by a vast class of dynamics, containing in particular all power traces of $L$. Note that the map $U_{+}^{-1} \circ \Phi^{-1}$ given by (5.45) differs from the identity solely by the shifts $-\Delta_{j}(\hat{p}) / 2$.

Let us now indicate how the above can be specialized to the $\mathrm{II}_{\mathrm{nr}}, \mathrm{I}_{\mathrm{rel}}$, and $\mathrm{I}_{\mathrm{nr}}$ systems. First, taking $\beta \rightarrow 0$, the matrix $\left(L-1_{N}\right) / \beta$ converges to 
the $\mathrm{II}_{\mathrm{nr}}$ Lax matrix (2.35), whereas the dual Lax matrix (5.33) converges to the (transpose of the) type $\mathrm{I}_{\text {rel }}$ Lax matrix, with $\beta$ replaced by $\mu$, and $\hat{p}_{j}$ and $\hat{x}_{j}$ playing the role of $x_{j}$ and $p_{j}$, respectively. Second, taking $\mu \rightarrow 0$, the matrix $L$ becomes the $\mathrm{I}_{\text {rel }}$ Lax matrix, whereas $\left(\hat{A}-1_{N}\right) / \mu$ converges to the $\mathrm{II}_{\mathrm{nr}}$ Lax matrix, with $\mu, x, p \rightarrow \beta, \hat{p}, \hat{x}$. Third, taking $\mu$ to 0 after the first limit, or $\beta$ to 0 after the second, one obtains the $\mathrm{I}_{\mathrm{nr}}$ matrices $L(x, p)$ and $L(\hat{p}, \hat{x})^{t}$ in a way that will be clear by now. Thus the duality properties (5.24) follow. Note that the shifts (5.42) and (5.43) vanish for the $\mathrm{I}_{\text {rel }}$ and $\mathrm{I}_{\mathrm{nr}}$ cases, whereas the $\mathrm{II}_{\mathrm{nr}}$ case yields a pair shift

$$
\delta(p)=\frac{1}{\mu} \ln \left(1+\frac{\mu^{2} g^{2}}{p^{2}}\right) .
$$

We continue by sketching how action-angle maps for the type III systems can be constructed, starting once more from the commutation relation (5.25). Of course, we should replace $\mu$ by $i \mu$ so as to obtain the relativistic type III Lax matrix. As it happens, the construction of the maps involves a lot more work than the construction of the $\mathrm{II}_{\text {rel }}$ map $\Phi$ detailed above. Therefore, we only mention some key points, referring for the details to Ref. 40 .

First, the commutation relation can be once more exploited to derive crucial spectral information: Fixing $(x, p)$ in the maximal type III phase space $\widetilde{\Omega}(2.24)$, the Lax matrix $L(x, p)$ satisfies

$$
\begin{aligned}
& L(x, p) \sim \operatorname{diag}\left(\exp \left(\beta \hat{p}_{1}\right), \ldots,\right.\left.\exp \left(\beta \hat{p}_{N}\right)\right), \\
& \hat{p}_{j}-\hat{p}_{j+1} \geq \mu g, \quad j=1, \ldots, N-1
\end{aligned}
$$

In words, its spectrum is not only positive and simple, but also has gaps. Points in $\widetilde{\Omega}$ for which one or more gaps are minimal correspond to partial equilibria. In particular, the set of points where all gaps are minimal is given by

$$
E=\left\{\begin{array}{l|l}
(x, p) \in \widetilde{\Omega} & \begin{array}{l}
x_{j}-x_{j+1}=2 \pi / N \mu, j=1, \ldots, N-1, \\
p_{k}=c, k=1, \ldots, N
\end{array}
\end{array}\right\} .
$$

For initial values in $E$ all of the commuting flows are of the form $(x(t), p(t))$ $=\left(x_{0}+c_{0}(t, \ldots, t), p_{0}\right)$; thus, all particles move uniformly along the line and no internal motion occurs. (Physically speaking, the classical molecule is in its ground state.)

A similar picture applies to the quotient phase spaces $\Omega^{\prime}$ and $\Omega$, since they may be coordinatized as subsets of $\widetilde{\Omega}$. Of course, the interpretation of the flows on (the quotients of) $E$ is now different: The $N$ particles rotate around the ring, keeping a fixed angular distance $2 \pi / N$ to nearest neighbors.

Deleting the points in the three phase spaces for which $L$ has one or more minimal spectral gaps, one is left with restricted phase spaces that serve 
as the definition domains of the respective action-angle maps. We continue by specializing to the "minimal' phase space $\Omega$ and its restriction $\Omega_{r}$. Then the action-angle map is of the form

$$
\Phi: \Omega_{r} \rightarrow \widehat{\Omega}=\mathbb{T}^{N} \times A_{N}, \quad(x, p) \mapsto(\hat{x}, \hat{p}),
$$

where

$$
A_{N} \equiv\left\{\hat{p} \in \mathbb{R}^{N} \mid \hat{p}_{j}-\hat{p}_{j+1}>\mu g, j=1, \ldots, N-1\right\} .
$$

The simplest dual dynamics is obtained by transforming

$$
\check{D}(x)=\frac{1}{2} \operatorname{Tr}\left(A(x)+A(x)^{*}\right)=\sum_{j=1}^{N} \cos \left(\mu x_{j}\right) .
$$

It reads

$$
D(\hat{x}, \hat{p})=\sum_{j=1}^{N} \cos \left(\mu \hat{x}_{j}\right) \prod_{k \neq j}\left(1-\frac{\operatorname{sh}^{2}(\beta \mu g / 2)}{\operatorname{sh}^{2}\left(\beta\left(\hat{p}_{j}-\hat{p}_{k}\right) / 2\right)}\right)^{1 / 2} \cdot\left(\hat{I I I ~}_{\mathrm{rel}}\right)
$$

From a physical point of view, the dual dynamics $D(p, x)$ describes $N$ particles on a line, whose distances $x_{j}-x_{j+1}$ are bounded below by $\mu g$, whereas their momenta $p_{j}$ vary over the first Brillouin zone $(-\pi / \mu, \pi / \mu]$. (Of course, the 1-tori might also be coordinatized by the interval $(-\pi, \pi]$, but then undesirable scale factors would crop up in the dual quantities.)

The flow generated by $D$ and the higher-power trace flows are not complete on $\widehat{\Omega}$. This is obvious from the fact that the corresponding flows on $\Omega$ (which are complete, of course) do not leave $\Omega_{r}$ invariant. However, it can be shown that the map $\Phi$ admits an extension to a symplectic map $\Phi^{\sharp}$ from $\langle\Omega, \omega\rangle$ onto a symplectic manifold $\left\langle\widehat{\Omega}^{\sharp}, \hat{\omega}^{\sharp}\right\rangle$ in which $\widehat{\Omega}$ is densely embedded, and on $\widehat{\Omega}^{\sharp}$ the flows are complete. We have dubbed this extension the harmonic oscillator transform, since it extends $\Phi$ in much the same way as the identity map in the example (5.3)-(5.7) extends the map (5.4) (when one views $\Phi$ as supplying new coordinates for the dense submanifold (5.5)). Thus, in the canonical variables coordinatizing $\widehat{\Omega}^{\sharp}$, the commuting flows have a trigonometric dependence on time (for the internal variables), and equilibria are no longer excluded.

As already mentioned, the dual flows on $\widehat{\Omega}^{\sharp}$ have a solitonic long-time asymptotics, but now this holds true only on an open dense unequal velocity subset that depends on the flow one selects. For instance, it is clear from (5.50) that the flow

$$
\exp (t \check{D})(x, p)=\left(x, p_{1}+\mu t \sin \mu x_{1}, \ldots, p_{N}+\mu t \sin \mu x_{N}\right)
$$

does not yield $\left|p_{j}(t)-p_{k}(t)\right| \rightarrow \infty$ for $j \neq k$ unless $\sin \mu x_{j} \neq \sin \mu x_{k}$, and when $x_{k}=\pi / \mu-x_{j}(\bmod 2 \pi / \mu)$ this condition is violated. A related complication is that, after deleting the equal velocity subvariety, one winds up 
with several connected components on which the ordering of the velocities is not the same.

The dynamics-dependent separatrices just described are reminiscent of the phenomenon mentioned in the last paragraph of Section 5.1. Indeed, the above setting yields a simple example for this phenomenon. The actionangle phase space $\Omega$ for the commuting Hamiltonians on $\widehat{\Omega}^{\sharp}$ is not of the product form (2.15), (2.16); only after discarding a suitable codimensionone subvariety it takes this form. For instance, when one deletes the variety $\left\{x_{1}=\pi / \mu\right\}$, one is left with a manifold that is the product of $\mathbb{R}^{N}$ and the interior of the set $F_{N}$ (2.28). (Geometrically, $\Omega$ can be viewed as a nontrivial fiber bundle: The base manifold equals $G / \Gamma_{x}$, where $G$ is the configuration space (2.24) and $\Gamma_{x}$ the restriction of the map $\Gamma$ (2.25) to $G$, the fiber equals $\mathbb{R}^{N}$, and the transition functions are cyclic permutations on the fiber.)

For the $\mathrm{III}_{\mathrm{b}}$ system described at the end of Section 3.2, a harmonic oscillator transform on the extended phase space $\mathbb{R}^{2} \times \mathbb{P}^{N-1}$ has been constructed as well [40]. This transform is self-dual, in the same sense as the $\mathrm{II}_{\mathrm{rel}}$ transform. Especially in the $\mathrm{III}_{\mathrm{b}}$ context, a new interpretation of the (analog of the) flip map $I$ (5.21) suggests itself. This reinterpretation is, however, also useful for the systems of type I-II already treated, and we proceed to describe it in that setting.

First, recall that the action variables play the role of positions for the dual systems. If we now agree to interchange the order of the factors in (2.15) and its various concretizations encountered above, then we no longer have any need for the flip map. (Though notational problems do remain: How should one choose notation making clear that the limits of $p(t)$ in (2.46) (for instance) are to be viewed as positions without creating confusion?) In particular, we are then free to identify $\Omega$ and $\widehat{\Omega}$ for the type I and II systems. Now this is acceptable, but it should be realized that there is a price to pay. One has

$$
\Phi^{*} \omega=-\omega
$$

the minus sign being caused by the flip. In words, $\Phi$ becomes an antisymplectomorphism of $\langle\Omega, \omega\rangle$.

Next, we define the involutory antisymplectomorphism

$$
\mathcal{T}(x, p) \equiv(x,-p)
$$

(time reversal) and the symplectomorphism

$$
\mathcal{F} \equiv \mathcal{T} \circ \Phi
$$

Now it is far from easy to see, but true, that one has

$$
\Phi \circ \mathcal{C}=\mathcal{T} \circ \Phi, \quad \Phi \circ \mathcal{T}=\mathcal{C} \circ \Phi,
$$


where $\mathcal{C}$ is the involutory antisymplectomorphism

$$
\mathcal{C}\left(x_{1}, \ldots, x_{N}, p_{1}, \ldots, p_{N}\right) \equiv\left(-x_{N}, \ldots,-x_{1}, p_{N}, \ldots, p_{1}\right) .
$$

(This follows by analytic continuation from results obtained in Ref. 40; cf. especially l.c. (2.118), (2.119).)

The point of the above is, that the symplectomorphism $\mathcal{F}$ on $\Omega$ transforms the flows generated by the Hamiltonians $H_{h}$ (5.35) into new flows on $\Omega$ that are linear in time. Specifically, using (5.38) one easily verifies

$$
\begin{aligned}
\left(\mathcal{F} \circ \exp \left(t H_{h}\right) \circ \mathcal{F}^{-1}\right) & (x, p) \\
& =\left(x, p_{1}-t h^{\prime}\left(-x_{1}\right), \ldots, p_{N}-t h^{\prime}\left(-x_{N}\right)\right) .
\end{aligned}
$$

The equations (5.52)-(5.55) hold true for each of the four type I and II systems, with (5.35) replaced by

$$
H_{h} \equiv \operatorname{Tr} h(L), \quad h \in C^{\infty}(\mathbb{R}), \quad\left(\mathrm{I}_{\mathrm{nr}}, \mathrm{II}_{\mathrm{nr}}\right)
$$

in the nonrelativistic case (where $\beta=0$ ).

Specializing now to the $\mathrm{II}_{\text {rel }}$ case, and taking $\beta=\mu$ so as to avoid scalings, we have additional information: Self-duality translates into $\Phi$ being an involution. From (5.53) and (5.54) we then deduce the relations

$$
\mathcal{F}^{-1}=\mathcal{P F}=\mathcal{F} \mathcal{P}=\mathcal{C} \mathcal{F C}=\mathcal{T} \mathcal{F} \mathcal{T}, \quad \mathcal{F}^{4}=\mathrm{id}(\Omega) \quad\left(\mathrm{II}_{\text {rel }}, \beta=\mu\right),
$$

where $\mathcal{P}$ (parity) is the involutory symplectomorphism

$$
\mathcal{P}\left(x_{1}, \ldots, x_{N}, p_{1}, \ldots, p_{N}\right) \equiv\left(-x_{N}, \ldots,-x_{1},-p_{N}, \ldots,-p_{1}\right) .
$$

These relations prepare us for the state of affairs on the quantum level, to which we now turn.

\section{Eigenfunction Transforms}

\subsection{Preliminaries}

As we have seen in Sections 4.2 and 4.3, the Poisson commuting Hamiltonians of the type I-VI systems admit quantizations as commuting PDOs and $A \Delta O$ s, respectively. This section is concerned with the eigenfunctions of these operators, especially inasmuch as these are relevant to the question whether the integrable PDOs and $A \Delta O$ s are Hilbert integrable--a notion introduced in Section 4.1. Before elaborating on this notion, let us delineate the PDOs and $\mathrm{A} \Delta \mathrm{O}$ s we intend to study.

First of all, we shall restrict attention to the systems of type I-IV. For information on joint eigenfunctions for Toda-type PDOs we refer to [54]. 
For the Toda-type A $\Delta \mathrm{O}$ (4.38) no eigenfunctions are known, and it may well be that these $A \Delta O$ s are not Hilbert integrable. (Note in this connection that they are not even formally symmetric. Nevertheless, it is conceivable that the enormous multiplier freedom for eigenfunctions of $\mathrm{A} \Delta \mathrm{O}$ s can be exploited to construct a unitary eigenfunction transform.)

Secondly, for type I-III we discuss the $N=2$ case in some detail, but we also present information on $N>2$ transforms, particularly for type IIi. At the relativistic level our starting point is formed by the commuting $\mathrm{A} \Delta \mathrm{Os}$ (4.35). Omitting the $\lambda$-dependent factor $c_{l}$ in (4.40) and expanding the resulting $\mathrm{A} \triangle \mathrm{O} A_{k}(\beta)$ according to (4.41), we obtain commuting PDOs $A_{1,1}, \ldots, A_{N, N}$ that will be our starting point at the nonrelativistic level.

Taking $\hbar \equiv 1$ from now on, the latter PDOs have the form

$$
\begin{gathered}
A_{1,1}=\sum_{j} \hat{p}_{j}, \quad A_{2,2}=\sum_{j<k}\left(\hat{p}_{j} \hat{p}_{k}-g(g-1) V\left(x_{j}-x_{k}\right)\right), \\
A_{k, k}=\sum_{i_{1}<\cdots<i_{k}} \hat{p}_{i_{1}} \cdots \hat{p}_{i_{k}}+\rho_{k}, \quad k>2,
\end{gathered}
$$

where $\rho_{k}$ has order $<k-1$ in $\hat{p}$. Also, $V(x)$ is given by (2.18)-(2.20), respectively. Recall that the change $g^{2} \rightarrow g(g-1)$ is a natural consequence of the nonrelativistic limit. (As will be seen, the eigenfunctions would have a quite awkward dependence on $g$ without this change.)

Thirdly, for type IV we consider again the A $\Delta \mathrm{O}$ s (4.35) at the relativistic level. Here, however, there is no obvious choice for the spectral parameter entering (4.40), and so we wind up with $\lambda$-dependent PDOs $A_{k, k}, k=1$, $\ldots, N$, when we take the nonrelativistic limit. In particular, $A_{2,2}$ is given by (4.43). We shall in fact restrict attention to the $N=2$ case. Indeed, eigenfunctions for $N>2$ are known only for $g=0,1$; observe in this connection that the A $\Delta$ Os are "free" for $g=1$, too; cf. (4.37), (4.34) with $g=\hbar$. (There are also some preliminary results for $N>2$ and $g=2,3$, ... in the nonrelativistic case [55].)

We now turn to some general remarks on the problem of proving Hilbert integrability for the commuting $\mathrm{PDO}$ and $\mathrm{A} \Delta \mathrm{O}$ s. Let us notice first that this problem has a distinctly analytic flavor-as opposed to the questions associated with the weaker notion of integrability, which are of an algebraic character. This parallels the situation at the classical level, where the completeness assumption defining Liouville integrability belongs to global analysis-as opposed to the local, algebraic notion of involutivity. In the classical setting, however, the extra requirement is quite easily verified for the models of type I-VI (as we have seen in Sections 2 and 3). By contrast, Hilbert integrability can only be verified by actually constructing a unitary joint eigenfunction transform - the quantum analog of the (inverse of the) action-angle transform.

A natural question is, therefore, whether there exists a more easily verified criterion from which the existence of such a diagonalizing transform 
would follow. For instance, one might try and show first that the pertinent operators have a well-defined action on a common dense invariant domain in $L^{2}(G, d x)$. But even when such a domain would exist, and the operators would commute and would be essentially self-adjoint on it, it would not follow that the corresponding time evolutions commute - which is necessary if a joint eigenfunction transform (as defined in Section 4.1) is to exist. (In case the operators are Hilbert integrable, a domain with all of the above properties does exist, viz., the subspace $\mathcal{E}\left(C_{0}^{\infty}(\Lambda)\right)$. This readily follows from Nelson's analytic vector theorem; cf. Ref. 43, p. 202.)

To get some feeling for what is involved here, we continue with some simple examples. First, we recall that the Fourier transform (4.7) diagonalizes both constant coefficient PDOs and the A $\Delta$ Os (4.8); cf. (4.9), (4.10). Specializing to the type I and II systems, we can use this transform to obtain free transforms of the form (4.11)-(4.13), as follows.

We begin by noting that wave functions belonging to the symmetric or antisymmetric subspaces $L_{s}^{2}\left(\mathbb{R}^{N}\right)$ and $L_{a}^{2}\left(\mathbb{R}^{N}\right)$, respectively, are uniquely determined by their restrictions to the wedge $G$ (2.23) (Weyl chamber). Obviously, $\mathcal{E}(4.7)$ intertwines the permutation operators (4.17) and their counterparts on $L^{2}\left(\mathbb{R}^{N}, d p\right)$, so it gives rise to unitary transforms

$$
\mathcal{E}_{s}, \mathcal{E}_{a}: L^{2}(G, d p) \rightarrow L^{2}(G, d x) .
$$

Explicitly, one obtains

$$
\begin{array}{ll}
\left(\mathcal{E}_{s} \phi\right)(x)=(2 \pi)^{-N / 2} \sum_{\sigma \in S_{N}} \int_{G} d p \exp (i p \cdot \sigma(x)) \phi(p), & x \in G, \\
\left(\mathcal{E}_{a} \phi\right)(x)=(2 \pi)^{-N / 2} \sum_{\sigma \in S_{N}}(-)^{\sigma} \int_{G} d p \exp (i p \cdot \sigma(x)) \phi(p), & x \in G .
\end{array}
$$

In this way we get two examples for a unitary of the form (4.11)-(4.13): $\Lambda$ equals $G(2.23), \mu$ equals Lebesgue measure, $M_{k}(p)$ in (4.13) is given by $e_{k}(p)(4.10), M_{1}(p)$ in (4.11) is given by $\sum_{j} p_{j}^{2} / 2$, and $M_{k}(p)$ in (4.11) depends, for $k>1$, on the choice of commuting PDOs $I_{2}, \ldots, I_{N}$. The domains of the two distinct self-adjoint operators on $L^{2}(G, d x)$ obtained via $\mathcal{E}_{s}$ and $\mathcal{E}_{a}$ (both associated with the PDO $H_{\mathrm{nr}}=\sum_{j} \hat{p}_{j}^{2} / 2$ ) then consist of restrictions to $G$ of symmetric and antisymmetric functions (respectively) in so-called Sobolev spaces. Similarly, the domains of the two self-adjoint operators associated via $\mathcal{E}_{s}\left(\mathcal{E}_{a}\right)$ with the $\mathrm{A} \Delta \mathrm{O} H_{\mathrm{rel}}=\sum_{j} \operatorname{ch}\left(\beta \hat{p}_{j}\right) / \beta^{2}$ consist of restrictions to $G$ of (anti)symmetric functions that admit analytic continuation to the polystrip $(\mathbb{R}+i(-\beta, \beta))^{N}$ and that have some further $L^{2}$-properties.

Due to the singularities of the potentials on the walls of $G$, the interacting Hamiltonians do not have a well-defined action on either of the two pertinent domains, however. For the same reason, it is not at all clear that a dense domain exists on which the interacting $H_{\text {rel }}$ is symmetric-as opposed to the interacting Hamiltonian $H_{\mathrm{nr}}$ (Schrödinger operator), which is 
symmetric on the dense subspace $C_{0}^{\infty}(G)$ (cf. the discussion at the end of Section 4.1).

Returning to the above free transforms, let us identify $L^{2}(G, d p)$ and $L^{2}(G, d x)$ via the antilinear map $f(p) \mapsto \overline{f(x)}$. This is the quantum version of the identification of $\Omega$ and $\widehat{\Omega}$ explained in the paragraph containing (5.52): It entails that $\mathcal{E}_{s}$ and $\mathcal{E}_{a}$ become antiunitary operators from $L^{2}(G, d x)$ onto itself. Introducing time reversal

$$
(\mathcal{T} \psi)(x) \equiv \overline{\psi(x)}, \quad \psi \in L^{2}(G, d x)
$$

parity

$$
(\mathcal{P} \psi)\left(x_{1}, \ldots, x_{N}\right) \equiv \psi\left(-x_{N}, \ldots,-x_{1}\right), \quad \psi \in L^{2}(G, d x),
$$

and the conjugation

$$
\mathcal{C} \equiv \mathcal{T P}
$$

it is then clear that one has

$$
\begin{gathered}
\mathcal{F}^{-1}=\mathcal{P} \mathcal{F}=\mathcal{F P}=\mathcal{C} \mathcal{F C}=\mathcal{T} \mathcal{F} \mathcal{T} \\
\mathcal{F}^{4}=1, \quad \mathcal{F} \equiv \mathcal{T} \mathcal{E}_{s}^{-1}, \mathcal{T} \mathcal{E}_{a}^{-1}
\end{gathered}
$$

This should be compared to (5.56).

We conjecture that for suitably restricted $g \geq 0$ there exists a unitary $\mathrm{II}_{\text {rel }}$ eigenfunction transform $\mathcal{E}_{g}$ given by (4.12), (4.13), with $\Lambda=G, \mu=d p$, $M_{k}=e_{k}(4.10)$, and $\mathcal{E}_{0}=\mathcal{E}_{s}, \mathcal{E}_{1}=\mathcal{E}_{a}$; with the above identification in effect, the unitary $\mathcal{F} \equiv \mathcal{T} \mathcal{E}_{g}^{-1}$ on $L^{2}(G, d x)$ obeys (6.3), provided $\beta=\mu$. To date, we have only shown this for $N=2$ and

$$
g \in\left[0,1+\frac{2 \pi}{\beta \mu}\right] .
$$

(We have also obtained some fragmentary results on eigenfunctions for $N>2$, but here the unitarity region is still unclear.) We shall present the pertinent $N=2$ eigenfunctions in Section 6.3. We intend to return to the corresponding transforms elsewhere.

\subsection{Type III Eigenfunctions for Arbitrary $N$}

We proceed by discussing eigenfunction transforms for the type III systems. Recall from Section 2.2 that one can distinguish three phase spaces for these systems, the choice depending on the physical interpretation. Accordingly, there are three state spaces at the quantum level, namely, the $L^{2}$-spaces over the sets $G, F_{N}^{\prime}$ and $F_{N}$; cf. (2.24)-(2.30).

We shall focus attention on the latter choice, which corresponds to indistinguishable particles on a ring. (Once the transforms on $L^{2}\left(F_{N}\right)$ are 
known, one can obtain transforms on $L^{2}\left(F_{N}^{\prime}\right)$ and $L^{2}(G)$ via suitable coordinate changes.) To ease the notation we take $\mu \equiv 1$ throughout this subsection.

Consider first the free case. Then we can exploit the well-known Fourier series orthonormal base for $L^{2}\left(\mathbb{T}^{N}\right) \simeq L^{2}\left((-\pi, \pi]^{N}, d x\right)$, viz., the functions

$$
K(x, n) \equiv(2 \pi)^{-N / 2} \exp (i x \cdot n), \quad x \in(-\pi, \pi]^{N}, n \in \mathbb{Z}^{N} .
$$

Specifically, the transform

$$
\mathcal{E}: l^{2}\left(\mathbb{Z}^{N}\right) \rightarrow L^{2}\left(\mathbb{T}^{N}\right), \quad \phi(n) \mapsto \sum_{n \in \mathbb{Z}^{N}} K(x, n) \phi(n),
$$

is a unitary operator that intertwines the permutation operators on the two Hilbert spaces involved, so it gives rise to two unitaries $\mathcal{E}_{s}$ and $\mathcal{E}_{a}$ mapping the symmetric and antisymmetric subspaces (respectively) of $l^{2}\left(\mathbb{Z}^{N}\right)$ onto those of $L^{2}\left(\mathbb{T}^{N}\right)$. Now the symmetric and antisymmetric functions in $L^{2}\left(\mathbb{T}^{N}\right)$ are uniquely determined by their restrictions to $F_{N}$; indeed, one has

$$
F_{N} \simeq \mathbb{T}_{\neq}^{N} / S_{N}, \quad \mathbb{T}_{\neq}^{N} \equiv\left\{x \in(-\pi, \pi]^{N} \mid x_{i} \neq x_{j}, 1 \leq i<j \leq N\right\} .
$$

That is, $F_{N}$ can be viewed as a fundamental set for the action of $S_{N}$ on $\mathbb{T}_{\not}^{N}$. Likewise,

$$
\mathbb{Z}_{+}^{N} \equiv\left\{n \in \mathbb{Z}^{N} \mid n_{N} \leq \cdots \leq n_{1}\right\}
$$

is a fundamental set for the $S_{N}$-action on $\mathbb{Z}^{N}$.

Specializing to the symmetric case first, we therefore obtain an orthonormal base

$$
M_{n}(x) \equiv r_{n} \sum_{\sigma \in S_{N}} \exp (i n \cdot \sigma(x)), \quad x \in F_{N}, n \in \mathbb{Z}_{+}^{N},
$$

for $L^{2}\left(F_{N}, d x\right)$, provided the normalization constant $r_{n}>0$ is suitably chosen. Equivalently, we obtain a unitary

$$
\mathcal{E}_{s}: l^{2}\left(\mathbb{Z}_{+}^{N}\right) \rightarrow L^{2}\left(F_{N}, d x\right), \quad \phi(n) \mapsto \sum_{n \in \mathbb{Z}_{+}^{N}} M_{n}(x) \phi(n)
$$

which obviously diagonalizes the free $\mathrm{A} \Delta \mathrm{O}$ s and PDOs.

Similarly, the antisymmetrized base functions $K(x, n)$ can be used. Specifically, we need here

$$
\mathbb{Z}_{+, \neq}^{N} \equiv\left\{n \in \mathbb{Z}^{N} \mid n_{N}<\cdots<n_{1}\right\}
$$

and the functions

$$
A_{n}(x) \equiv r \sum_{\sigma \in S_{N}}(-)^{\sigma} \exp (i n \cdot \sigma(x)), \quad x \in F_{N}, n \in \mathbb{Z}_{+, \neq}^{N},
$$


to obtain an orthonormal base for $L^{2}\left(F_{N}, d x\right)$ (for a suitable constant $r>$ $0)$. Hence we get a unitary

$$
\mathcal{E}_{a}: l^{2}\left(\mathbb{Z}_{+, \neq}^{N}\right) \rightarrow L^{2}\left(F_{N}, d x\right), \quad \phi(n) \mapsto \sum_{n \in \mathbb{Z}_{+, \neq}^{N}} A_{n}(x) \phi(n)
$$

which also diagonalizes the free $\mathrm{A} \Delta \mathrm{O}$ s and PDOs.

With these elementary examples and the associated notation at our disposal, we can now turn to a description of the $g \geq 0$ type III transforms $\mathcal{E}_{g}$. The pertinent eigenfunctions were introduced (in somewhat different guises) in Refs. 14 and 15 for $\beta=0$ and in Ref. 16 for $\beta>0$; cf. also Ref. 17 . Not surprisingly, one has $\mathcal{E}_{0}=\mathcal{E}_{s}$ for all $N$, but $\mathcal{E}_{1}$ is only equal to $\mathcal{E}_{a}$ for $N$ odd. Of course, $\mathcal{E}_{1}$ is a free transform for $N$ even, too, but in this case it is the antisymmetric reduction of the Fourier base for $L^{2}\left(\mathbb{T}^{N}\right)$ with antiperiodic boundary conditions, i.e., the functions (6.4) with $n \in(\mathbb{Z}+1 / 2)^{N}$.

To handle the general case, it is convenient to introduce the vector

$$
\rho(g) \equiv \frac{g}{2}(N-1, N-3, \ldots,-N+1)
$$

and the set

$$
\Lambda_{g} \equiv\left\{p \in \mathbb{R}^{N} \mid p-\rho(g) \in \mathbb{Z}_{+}^{N}\right\}
$$

Then the transforms are unitaries of the form

$$
\mathcal{E}_{g}: l^{2}\left(\Lambda_{g}\right) \rightarrow L^{2}\left(F_{N}, d x\right), \quad \phi(p) \mapsto \sum_{p \in \Lambda_{g}} E(x, p) \phi(p) .
$$

Here, the $\beta$-dependence is suppressed; in the relativistic case $\beta>0$ the kernel $E(x, p)$ is a joint eigenfunction of the $\mathrm{A} \Delta \mathrm{O}$ s (4.35), satisfying

$$
S_{ \pm k} E(x, p)=\sum_{|I|=k} \exp \left( \pm \beta p_{I}\right) E(x, p), \quad k=1, \ldots, N
$$

and taking $\beta \rightarrow 0$ one then deduces that for the PDOs (6.1), (6.2) one has

$$
A_{k, k} E(x, p)=\sum_{i_{1}<\cdots<i_{k}} p_{i_{1}} \cdots p_{i_{k}} E(x, p) .
$$

As a consequence, the type III $\mathrm{A} \Delta \mathrm{O}$ s and PDOs are Hilbert integrable: (4.11)-(4.13) hold true, with $\mu$ the counting measure having support on $\Lambda_{g}$.

The eigenfunctions $E(x, p)$ can be written

$$
E(x, p)=\Delta(x)^{1 / 2} P_{p-\rho(g)}(x), \quad x \in F_{N}, p \in \Lambda_{g}
$$

Using a tilde from now on to denote equality up to a positive constant, $\Delta(x)$ is of the form

$$
\Delta(x) \sim \prod_{\substack{j, k=1 \\ j<k}}^{N} w\left(x_{j}-x_{k}\right)
$$


Furthermore, $P_{n}(x), n \in \Lambda_{0}$, is a finite linear combination of the above polynomials $M_{n}(x)$. We continue by providing more details on the weight function $w(x)$ and the linear combination involved.

For $\beta>0$ the $w$-function is proportional to an infinite product, namely,

$$
w(x) \sim \prod_{l=0}^{\infty} \frac{(1-\exp [i x-l \beta])(x \rightarrow-x)}{(1-\exp [i x-(l+g) \beta])(x \rightarrow-x)}
$$

while for $\beta=0$ one has

$$
w(x) \sim\left|\sin \frac{x}{2}\right|^{2 g} . \quad(\beta=0)
$$

The $w$-function (6.10) is the trigonometric degeneration of the elliptic $w$ function from Ref. [31], which was used in Section 4.3; cf. (4.44)-(4.46). As such, the function $\Delta(x)(6.9)$ gives rise to $\mathrm{A} \Delta \mathrm{Os} S_{k}^{t}$ of the form (4.46). (This is easily verified directly.) On account of (6.6) and (6.8), these $A \Delta O \mathrm{~s}$ satisfy

$$
S_{k}^{t} P_{n}(x)=\sum_{|I|=k} \exp \left(\beta \sum_{i \in I}\left(n_{i}+\rho(g)_{i}\right)\right) P_{n}(x), \quad n \in \Lambda_{0},
$$

i.e., they admit multivariable polynomials as joint eigenfunctions. Taking $\beta \rightarrow 0$, this holds true for the correspondingly transformed PDOs $A_{k, k}^{t}$, too (recall (4.45), (4.47), and (4.48) in this connection).

Let us now describe the structure of the polynomials $P_{n}(x)$ in more detail. To this end we need a partial order on the set $\Lambda_{0}=\mathbb{Z}_{+}^{N}$ :

$$
\begin{aligned}
n & \leq m \\
& \Longleftrightarrow \sum_{j=1}^{N}\left(n_{j}-m_{j}\right)=0, \quad \sum_{j=1}^{k}\left(n_{j}-m_{j}\right) \leq 0, k=1, \ldots, N-1 .
\end{aligned}
$$

Thus, for a fixed $m \in \Lambda_{0}$ there are only finitely many $n \in \Lambda_{0}$ satisfying $n \leq m$; in particular, for $m_{j}$ of the form $(j, \ldots, j)$ there is only one such $n$, namely $n=m_{j}$. The polynomials are now of the triangular form

$$
P_{m}(x)=\sum_{n \leq m} c_{m n} M_{n}(x), \quad c_{m m}>0
$$

with coefficients $c_{m n}$ depending on $\beta$ and $g$.

Before explaining how the coefficients $c_{m n}$ are determined, we insert a remark on the (in)significance of fractional statistics for the eigenfunctions $E(x, p)(6.8)$ at hand. Obviously, the polynomials $P_{m}(x), x \in F_{N}$, extend to functions that are entire and symmetric in $x_{1}, \ldots, x_{N}$, both in the 
nonrelativistic $(\beta=0)$ and the relativistic $(\beta>0)$ case. Now consider the factor (cf. (6.9), (6.11))

$$
\Delta(x)^{1 / 2} \sim \prod_{j<k} \sin \frac{1}{2}\left(x_{j}-x_{k}\right)^{g}, \quad x \in F_{N} . \quad(\beta=0)
$$

We have omitted the bars in (6.11), since all of the sines occurring in (6.15) are positive on $F_{N}(2.28)$. Clearly, the function at the right-hand side (and hence $E(x, p)$ as well) extends to a function that is entire and symmetric (antisymmetric) in $x_{1}, \ldots, x_{N}$ for $g$ even (odd), whereas it has logarithmic branch points on the walls of the Weyl alcove $F_{N}$ for noninteger $g$. This is the so-called fractional statistics phenomenon: The particles are viewed as bosons (fermions) for $g$ even (odd), and as anyons for noninteger $g$.

We would like to point out that this state of affairs is an artefact of the nonrelativistic limit. For $\beta>0$ and $g>0$ one gets from (6.10) (by splitting off the $l=0$ numerator factor at the right-hand side)

$$
\Delta(x)^{1 / 2} \sim \prod_{j<k} \sin \frac{1}{2}\left(x_{j}-x_{k}\right) w_{r}\left(x_{j}-x_{k}\right)^{1 / 2}, \quad x \in F_{N}, \quad(\beta>0)
$$

where $w_{r}(x)$ extends to a meromorphic, even function that is positive on $\mathbb{R}$. (In fact, from $(6.10)$ one reads off that $w_{r}(x)$ has no zeros and poles in the strip $|\operatorname{Im} x|<\min (\beta, g)$.) As a consequence, no trace of fractional statistics remains: For all $g>0$ the particles are fermions. (Of course, for $g=0$ the function $E(x, p), x \in F_{N}$, extends to a symmetric function; there are noncommuting operations involved here.)

A similar change of anyons to fermions occurs for the $\beta>0$ eigenfunctions of type II-inasmuch as these are known. It is caused by the same phenomenon as for type III: fixing $g \notin \mathbb{N}$, the (meromorphic) $w$-function has an infinite number of poles and zeros coalescing on the imaginary axis for $\beta \downarrow 0$ [31].

We continue by explaining how the coefficients in (6.14) are determined. To this end we introduce the renormalized polynomials

$$
\begin{gathered}
Q_{m}(x) \equiv \frac{P_{m}(x)}{c_{m m}}=M_{m}(x)-R_{m}(x), \\
R_{m}(x)=\sum_{n<m} d_{m n} M_{n}(x), \quad d_{m n} \equiv-\frac{c_{m n}}{c_{m m}},
\end{gathered}
$$

the point being that $R_{m}(x)$ equals the orthogonal projection of $M_{m}(x)$ onto the subspace

$$
\mathcal{H}_{m} \equiv\left\{\sum_{n<m} c_{n} M_{n} \mid c_{n} \in \mathbb{C}\right\}
$$

in the Hilbert space $L^{2}\left(F_{N}, \Delta(x) d x\right)$. This determines the coefficients $d_{m n 2}$ uniquely, and then $c_{m m}$ is determined by requiring that $P_{m}(x)$ be a unit 
vector in the latter Hilbert space. In particular, for $g=0$ the function $\Delta(x)$ reduces to a positive constant (cf. (6.9)-(6.11)), and so $R_{m}(x)$ vanishes. Hence one obtains $\mathcal{E}_{0}=\mathcal{E}_{s}$, as announced above.

It is obvious from the previous paragraph that $\Delta(x)$ uniquely determines the polynomials $Q_{m}(x)$, and that one has

$$
\int_{F_{N}} d x \Delta(x) \bar{Q}_{m}(x) Q_{n}(x)=0, \quad n<m .
$$

However, this much is true for a quite arbitrary weight function. Indeed, for $N=2$ and $\Delta(x) \equiv f\left(x_{1}-x_{2}\right)$ with $f(x)$ any positive, continuous and even function on $[-2 \pi, 2 \pi]$, the above characterization yields an orthonormal base. (It amounts to the well-known Gram-Schmidt procedure.) But for $N>2$ the vectors $M_{n}$ in the subspace $\mathcal{H}_{m}(6.16)$ are not totally ordered. (For example, taking

$$
m=(5,4,0), \quad n_{1}=(5,2,2), \quad n_{2}=(4,4,1),
$$

one has $n_{1}<m$ and $n_{2}<m$, yet neither $n_{1}<n_{2}$ nor $n_{2}<n_{1}$ holds true.) The above weight functions have the (very restrictive) property that (6.17) holds not only for $n<m$ but for all pairs $n \neq m$.

We proceed by sketching how the (transformed) A $\triangle$ Os and PDOs can be used to prove this orthogonality property; in the process it will become clear why $Q_{m}$ is a joint eigenfunction. Let us denote a fixed operator among the A $\triangle$ Os or PDOs by $A$. Each of the operators has two key properties. First, it satisfies

$$
A M_{m}=E_{m} M_{m}+\sum_{n<m} \alpha_{m n} M_{n}
$$

That is, it is triangular w.r.t. the partial order (6.13) on the free boson eigenstates $M_{m}$. Second, it is symmetric on the dense subspace of $L^{2}\left(F_{N}, \Delta(x) d x\right)$ spanned by these functions; this amounts to

$$
\left(A M_{m}, M_{n}\right)=\left(M_{m}, A M_{n}\right), \quad \forall m, n \in \Lambda_{0},
$$

where $(\cdot, \cdot)$ denotes the scalar product in $L^{2}\left(F_{N}, \Delta(x) d x\right)$.

These two features, combined with the above definition of the polynomials $Q_{m}$, entail that $Q_{m}$ is an eigenfunction of $A$ with eigenvalue $E_{m}$. Indeed, by virtue of triangularity of $A$ and $Q_{m}$ one obtains

$$
A Q_{m}=E_{m} Q_{m}+\sum_{n<m} a_{m n} Q_{n} \equiv E_{m} Q_{m}+L_{m}
$$

so it suffices to show $L_{m}=0$. Now (6.17) entails $\left(Q_{m}, L_{m}\right)=0$ and so one infers (using also symmetry of $A$ )

$$
\begin{aligned}
\left(L_{m}, L_{m}\right) & =\left(A Q_{m}, L_{m}\right)=\left(Q_{m}, A L_{m}\right)=\sum_{n<m} a_{m n}\left(Q_{m}, A Q_{n}\right) \\
& =\sum_{n<m} a_{m n} \sum_{j \leq n} a_{n j}\left(Q_{m}, Q_{j}\right)=0
\end{aligned}
$$


since $j \leq n<m$. Therefore, $L_{m}$ vanishes.

The upshot is that the eigenfunction property of the polynomials $Q_{m}$ has been reduced to the triangularity and symmetry relations (6.18) and (6.19). Taking these relations for granted-their proofs are not difficult, but would take us too far afield -it is not hard to see that the eigenvalues $E_{m}$ are in fact given by (6.12) in the $\mathrm{A} \Delta \mathrm{O}$ case and, therefore, by (6.7) in the PDO case. Fixing a pair $m \neq n$, we shall now show how this eigenvalue structure entails the announced orthogonality $\left(Q_{m}, Q_{n}\right)=0$.

Clearly, we need only prove that at least one of the A $\triangle$ Os or PDOs $A_{k}$, $k=1, \ldots, N$, yields eigenvalues $E_{m}^{(k)} \neq E_{n}^{(k)}$ on $Q_{m}$ and $Q_{n}$, respectively. (Indeed, orthogonality then follows from symmetry in a well-known way.) Let us assume all eigenvalues are equal. Now the eigenvalues are the symmetric functions of a matrix of the form $\operatorname{diag}\left(\lambda_{1}, \ldots, \lambda_{N}\right)$ with $\lambda_{N}<\cdots<\lambda_{1}$. But then equality of all eigenvalues entails that the two vectors $\lambda(m), \lambda(n)$ involved are equal, so that $m=n$, a contradiction.

With orthogonality established, one need only normalize to obtain the orthonormal base $\left\{P_{n}(x)\right\}_{n \in \Lambda_{0}}$ of $L^{2}\left(F_{N}, \Delta(x) d x\right)$ and the corresponding orthonormal base $\{E(x, p)\}_{p \in \Lambda_{g}}$ of $L^{2}\left(F_{N}, d x\right)$. Note that the argument in the previous paragraph not only proves orthogonality, but also-using standard quantum-mechanical parlance-completeness of the set of observables $\left\{A_{1}, \ldots, A_{N}\right\}$. Rephrased in functional analytic language, the von Neumann algebra, generated by the bounded functions of the self-adjoint operators on $L^{2}\left(F_{N}, d x\right)$ associated with $A_{1}, \ldots, A_{N}$ via the unitary $\mathcal{E}_{g}$, is maximal Abelian.

The crucial triangularity property (6.18) was first observed and exploited by Sutherland $[14,36]$, who used the PDO $A=H_{\mathrm{nr}}^{t}=\left(A_{1,1}^{t}\right)^{2} / 2-A_{2,2}^{t}$. For $\beta>0$ the A $\Delta$ Os $S_{k}^{t}$ and the polynomials $Q_{m}$ were first introduced by Macdonald [16, 17]. To be more specific, he considers several root systems at once and accordingly works, with the center-of-mass versions of the $S_{k}^{t}$ and $Q_{m}$; for the detailed relation we refer to Section 5.2 of Ref. 6 .

We conclude this subsection by pointing out some illuminating relations between the classical and quantum diagonalizing transforms. First, denoting the closure of the range of variation $A_{N}$ (5.49) of the actions by $A_{N}^{c l}$, the set $\Lambda_{g}(6.5)$ is a lattice-type subset of $A_{N}^{c l}$, whose boundary points (vectors $p$ for which $p_{j}-p_{j+1}=g$ for some $j$ ) are also boundary points of $A_{N}^{c l}$ (partial equilibria). Moreover, the eigenvalues of the quantum Hamiltonians on a joint eigenfunction $E(x, p)$ are obtained by evaluating their classical counterparts in points of $\Omega$ whose action vector equals $p \in \Lambda_{g} \subset A_{N}^{c l}$. (Semiclassical quantization is exact.)

Second, in agreement with the correspondence just sketched, the quantum ground state is obtained by choosing $p=\rho(g)$; cf. (6.8). (This choice yields the minimal eigenvalue for the defining Hamiltonians, as is readily verified.) In fact, the triangular structure (6.14) entails

$$
P_{m_{0}}(x)=c_{0}>0, \quad m_{0} \equiv(0, \ldots, 0),
$$


so that the ground state reads explicitly

$$
E(x, \rho(g))=c_{0} \Delta(x)^{1 / 2} .
$$

Third, the joint eigenfunction property of $P_{m_{0}}$ can be translated into a set of $N$ functional equations. Indeed, recalling (4.46) and the definition of $f_{-}(x)^{2}$ (cf. (4.34) and (2.65)), we see that (6.12) with $n=m_{0}$ amounts to

$$
\sum_{\substack{I \subset\{1, \ldots, N\} \\|I|=k}} \prod_{\substack{j \in I \\ l \notin I}} \frac{\sin \left(x_{j}-x_{l}-i \beta g\right) / 2}{\sin \left(x_{j}-x_{l}\right) / 2}=\sum_{\substack{I \subset\{1, \ldots, N\} \\|I|=k}} \exp \left(\frac{1}{2} \beta g \sum_{j \in I}(N+1-2 j)\right) .
$$

These functional equations can also be proved directly; cf. Lemma A.5 in Ref. 40 . In the classical context they are exploited to prove that the type III Lax matrix has minimal spectral gaps on the set $E$ (5.48). Consequently, they encode both the classical and the quantum ground-state properties; the quantum ground-state (6.20) corresponds to the classical equilibrium subset $E_{0}$ of $E$ obtained by putting $c=0$ at the right-hand side of (5.48).

\subsection{Type II and IV Eigenfunctions for $N=2$}

From now on we specialize to the $N=2$ case. It is convenient to separate off the center-of-mass motion by employing new coordinates

$$
\begin{array}{ll}
X \equiv \frac{x_{1}+x_{2}}{2}, & P \equiv p_{1}+p_{2}, \\
x \equiv x_{1}-x_{2}, & p \equiv \frac{p_{1}-p_{2}}{2} .
\end{array}
$$

Then the dependence of the eigenfunctions on the center-of-mass coordinates $X, P$ and internal coordinates $x, p$ can be factorized:

$$
E_{2}\left(\left(x_{1}, x_{2}\right),\left(p_{1}, p_{2}\right)\right)=\exp (i X P) E(x, p) .
$$

Indeed, in this way we obtain an eigenfunction of the A $\Delta$ Os $S_{ \pm 2}$ and PDO $A_{1,1}$ with eigenvalues $\exp ( \pm \beta P)$ and $P$, respectively, and it remains to consider the operators $S_{ \pm 1}$ and $A_{2,2}(6.1)$.

Specializing first to the type II case, we restrict the choice of the spectral variables $p_{1}, p_{2}$ by insisting that for $\beta>0$ the $\mathrm{A} \Delta \mathrm{Os} S_{ \pm 1}$ yield eigenvalues

$$
\exp \left( \pm \beta p_{1}\right)+\exp \left( \pm \beta p_{2}\right)=2 \exp \left( \pm \beta \frac{P}{2}\right) \operatorname{ch}(\beta p)
$$

and that for $\beta=0$ the $\mathrm{PDO} A_{2,2}$ yields eigenvalue

$$
p_{1} p_{2}=\frac{P^{2}}{4}-p^{2}
$$


on $E_{2}$ (6.21). Using the new parameter $\nu \equiv \mu / 2$ (which minimizes the occurrence of numerical factors), it follows that the function $E(x, p)$ at the right-hand side of (6.21) should satisfy

$$
\begin{gathered}
\left(\frac{\operatorname{sh} \nu(x-i \beta g) \operatorname{sh} \nu(x-i \beta+i \beta g)}{\operatorname{sh} \nu x \operatorname{sh} \nu(x-i \beta)}\right)^{1 / 2} E(x-i \beta, p)+(\beta \rightarrow-\beta) \\
=2 \operatorname{ch}(\beta p) E(x, p), \\
-\partial_{x}^{2} E(x, p)+g(g-1) \frac{\nu^{2}}{\operatorname{sh}^{2} \nu x} E(x, p)=p^{2} E(x, p),
\end{gathered}
$$

for $\beta>0$ and $\beta=0$, respectively.

We write the elliptic generalizations of the operators at the left-hand sides as

$$
\begin{aligned}
& H_{\mathrm{rel}}=\left(\frac{s(x-i \beta g)}{s(x)}\right)^{1 / 2} T_{i \beta}^{x}\left(\frac{s(x+i \beta g)}{s(x)}\right)^{1 / 2}+(\beta \rightarrow-\beta) \\
& H_{\mathrm{nr}}=-\frac{d^{2}}{d x^{2}}+g(g-1)\left(\mathcal{P}(x)+\frac{\eta}{\omega}\right)
\end{aligned}
$$

Here, we have introduced the shift

$$
\left(T_{\xi}^{x} f\right)(x) \equiv f(x-\xi), \quad \xi \in \mathbb{C}
$$

and we are discarding a $\lambda$-dependent constant in (4.43). In this case there is no obvious parametrization for eigenvalues, as will be seen below. Just as for the type III case considered in Section 6.2 , it is in fact more convenient to work with the measure $w(x) d x$ instead of Lebesgue measure $d x$, where $w(x)$ is the $w$-function that already appeared in Section 4.3; cf. (4.44), (4.45). After the corresponding similarity transformation, one obtains from (6.22) and (6.23) the operators (recall (4.46)-(4.48))

$$
\begin{aligned}
& H_{\mathrm{rel}}^{t}=\frac{s(x-i \beta g)}{s(x)} T_{i \beta}^{x}+(\beta \rightarrow-\beta), \\
& H_{\mathrm{nr}}^{t}=-\frac{d^{2}}{d x^{2}}-2 g \frac{s^{\prime}(x)}{s(x)} \frac{d}{d x}-g \frac{s^{\prime \prime}(x)}{s(x)}+g(g-1)\left(\mathcal{P}(x)+\frac{\eta}{\omega}-\frac{s^{\prime}(x)^{2}}{s(x)^{2}}\right) .
\end{aligned}
$$

For the hyperbolic specialization,

$$
\begin{aligned}
& H_{\mathrm{rel}}^{t}=\frac{\operatorname{sh} \nu(x-i \beta g)}{\operatorname{sh} \nu x} T_{i \beta}^{x}+(\beta \rightarrow-\beta), \\
& H_{\mathrm{nr}}^{t}=-\frac{d^{2}}{d x^{2}}-2 g \nu \operatorname{coth} \nu x \frac{d}{d x}-g^{2} \nu^{2},
\end{aligned}
$$

the dual operators expected from the classical level read (recall (5.24))

$$
\begin{aligned}
\widehat{H}_{\mathrm{rel}}^{t} & =\frac{\operatorname{sh} \beta(p-i \nu g)}{\operatorname{sh} \beta p} T_{i \nu}^{p}+(\nu \rightarrow-\nu), \\
\widehat{H}_{\mathrm{nr}}^{t} & =\frac{p-i \nu g}{p} T_{i \nu}^{p}+(\nu \rightarrow-\nu) .
\end{aligned}
$$


More specifically, writing

$$
E(x, p) \equiv C w(x)^{1 / 2} R(x, p) \hat{w}(p)^{1 / 2}
$$

(where $C>0$ is a normalization constant), one should have

$$
\begin{aligned}
H_{\mathrm{rel}}^{t} R(x, p) & =2 \operatorname{ch}(\beta p) R(x, p), & \widehat{H}_{\mathrm{rel}}^{t} R(x, p) & =2 \operatorname{ch}(\nu x) R(x, p), \\
H_{\mathrm{nr}}^{t} R(x, p) & =p^{2} R(x, p), & \widehat{H}_{\mathrm{nr}}^{t} R(x, p) & =2 \operatorname{ch}(\nu x) R(x, p) .
\end{aligned}
$$

In our survey [5] we have already pointed out that this expectation is satisfied in the nonrelativistic case. Indeed, here one has

$$
R(x, p)={ }_{2} F_{1}\left(\frac{1}{2}\left(g+i \frac{p}{\nu}\right), \frac{1}{2}\left(g-i \frac{p}{\nu}\right), g+\frac{1}{2} ;-\operatorname{sh}^{2} \nu x\right)
$$

cf. l.c. (3.35), and the dual equation follows from the contiguous relations for the Gauss hypergeometric function ${ }_{2} F_{1}$. In l.c. (3.37) (3.42), we have also presented solutions to (6.28) with all of the expected properties, taking, however, $g \in \mathbb{N}$.

The general solution (which we have obtained in recent years) reduces to this special case for $g \in \mathbb{N}$, but it has a quite different appearance. As mentioned in the introduction to this chapter, its structure is actually such as to admit a straightforward generalization to a simultaneous eigenfunction for four Askey-Wilson-type hyperbolic A $\Delta \mathrm{Os}$, each of which depends on four coupling constants. We shall describe these $\mathrm{A} \Delta \mathrm{O}$ s and the general solution first, using notation that is convenient to bring out the symmetry properties, and then detail the pertinent specializations. We write the four couplings involved as

$$
\mathbf{c}=\left(c_{0}, c_{1}, c_{2}, c_{3}\right) .
$$

It is convenient to introduce matrices

$$
I \equiv\left(\begin{array}{llll}
1 & 0 & 0 & 0 \\
0 & 0 & 1 & 0 \\
0 & 1 & 0 & 0 \\
0 & 0 & 0 & 1
\end{array}\right), \quad J \equiv \frac{1}{2}\left(\begin{array}{cccc}
1 & 1 & 1 & 1 \\
1 & 1 & -1 & -1 \\
1 & -1 & 1 & -1 \\
1 & -1 & -1 & 1
\end{array}\right)
$$

satisfying

$$
I J=J I, \quad K=K^{*}=K^{-1}, K=I, J,
$$

and dual couplings given by

$$
\hat{\mathbf{c}} \equiv J \mathbf{c}=\left(\hat{c}_{0}, \hat{c}_{1}, \hat{c}_{2}, \hat{c}_{3}\right) .
$$

To define the four $\mathrm{A} \Delta \mathrm{O}$ s, we use the functions

$$
s_{\delta}(z) \equiv \operatorname{sh}\left(\frac{\pi z}{a_{\delta}}\right), \quad c_{\delta}(z) \equiv \operatorname{ch}\left(\frac{\pi z}{a_{\delta}}\right), \quad \delta=+,-, a_{+}, a_{-}>0,
$$


and the shift (6.24). Now we introduce the $\mathrm{A} \Delta \mathrm{O}$

$$
A_{\delta}(\mathbf{c} ; z) \equiv C_{\delta}(z)\left(T_{i a_{-} \delta}^{z}-1\right)+C_{\delta}(-z)\left(T_{-i a_{-} \delta}^{z}-1\right)+2 c_{\delta}\left(2 i \hat{c}_{0}\right), \delta=+,-,
$$

where

$$
C_{\delta}(z) \equiv \frac{s_{\delta}\left(z-i c_{0}\right)}{s_{\delta}(z)} \frac{c_{\delta}\left(z-i c_{1}\right)}{c_{\delta}(z)} \frac{s_{\delta}\left(z-i c_{2}-i a_{-\delta} / 2\right)}{s_{\delta}\left(z-i a_{-\delta} / 2\right)} \frac{c_{\delta}\left(z-i c_{3}-i a_{-\delta} / 2\right)}{c_{\delta}\left(z-i a_{-\delta} / 2\right)}
$$

The function $R$ we are about to introduce is a joint eigenfunction of the $\mathrm{A} \Delta \mathrm{Os}$

$$
A_{+}(\mathbf{c} ; v), \quad A_{-}(I \mathbf{c} ; v), \quad A_{+}(\hat{\mathbf{c}} ; \hat{v}), \quad A_{-}(I \hat{\mathbf{c}} ; \hat{v})
$$

with eigenvalues

$$
2 c_{+}(2 \hat{v}), \quad 2 c_{-}(2 \hat{v}), \quad 2 c_{+}(2 v), \quad 2 c_{-}(2 v),
$$

respectively. (Note in this connection that the two $\mathrm{A} \Delta \mathrm{O}$ s acting on $v$ or on $\hat{v}$ commute; cf. also the paragraph containing (4.14).) It is given by an integral involving products of the function

$$
G\left(a_{+}, a_{-} ; z\right) \equiv \exp \left(i \int_{0}^{\infty} \frac{d y}{y}\left(\frac{\sin 2 y z}{2 \operatorname{sh}\left(a_{+} y\right) \operatorname{sh}\left(a_{-} y\right)}-\frac{z}{a_{+} a_{-} y}\right)\right)
$$

where $|\operatorname{Im} z|<\frac{1}{2}\left(a_{+}+a_{-}\right)$. This building block is studied in detail in Section 3.1 of Ref. 31 . The $G$-function extends to a meromorphic function that satisfies three elementary first-order analytic difference equations. It may be viewed as a generalization of the gamma function, and the integral representation for the eigenfunction $R$ may be viewed as a generalization of the Barnes representation for the hypergeometric function.

The $G$-function is manifestly symmetric in $a_{+}, a_{-}$; we shall suppress these parameters. Taking from now on

$$
\begin{gathered}
v, \hat{v}, c_{0}, \hat{c}_{0}>0, c_{0}+c_{1}<\frac{1}{2} a_{+}+a_{-}, \\
c_{0}+c_{2}<a_{+}+\frac{1}{2} a_{-}, c_{0}+c_{3}<\frac{1}{2}\left(a_{+}+a_{-}\right)
\end{gathered}
$$

(this restriction is imposed to ease the exposition), we define

$$
R\left(a_{+}, a_{-}, \mathbf{c} ; v, \hat{v}\right) \equiv\left(a_{+} a_{-}\right)^{-1 / 2} \int_{\Gamma} d z I\left(a_{+}, a_{-}, \mathbf{c} ; v, \hat{v}, z\right)
$$

where $\Gamma$ is a contour along the real axis, indented downward near the origin so as to avoid a simple pole. The integrand is given by

$$
I \equiv F\left(c_{0} ; v, z\right) K\left(a_{+}, a_{-}, \mathbf{c} ; z\right) F\left(\hat{c}_{0} ; \hat{v}, z\right) .
$$


The function $F$ is symmetric in $a_{+}, a_{-}$:

$$
F(c ; y, z) \equiv\left(\frac{G\left(y+z+i c-i\left(a_{+}+a_{-}\right) / 2\right)}{G\left(y+i c-i\left(a_{+}+a_{-}\right) / 2\right)}\right)(y \rightarrow-y) .
$$

By contrast, the kernel function $K$ is not symmetric:

$$
\begin{array}{r}
K\left(a_{+}, a_{-}, \mathbf{c} ; z\right) \equiv \frac{1}{G\left(z+i\left(a_{+}+a_{-}\right) / 2\right)} \frac{G\left(i\left(c_{0}+c_{1}\right)-i a_{-} / 2\right)}{G\left(z+i\left(c_{0}+c_{1}\right)-i a_{-} / 2\right)} \\
\cdot \frac{G\left(i\left(c_{0}+c_{2}\right)-i a_{+} / 2\right)}{G\left(z+i\left(c_{0}+c_{2}\right)-i a_{+} / 2\right)} \frac{G\left(i\left(c_{0}+c_{3}\right)\right)}{G\left(z+i\left(c_{0}+c_{3}\right)\right)} .
\end{array}
$$

However, it satisfies

$$
K\left(a_{+}, a_{-}, \mathbf{c} ; z\right)=K\left(a_{-}, a_{+}, I \mathbf{c} ; z\right)
$$

which entails

$$
R\left(a_{+}, a_{-}, \mathbf{c} ; v, \hat{v}\right)=R\left(a_{-}, a_{+}, I \mathbf{c} ; v, \hat{v}\right) .
$$

It is also clear from the above that one has

$$
\begin{aligned}
& R\left(a_{+}, a_{-}, \mathbf{c} ; v, \hat{v}\right)=R\left(a_{+}, a_{-}, \hat{\mathbf{c}} ; \hat{v}, v\right), \\
& R\left(a_{+}, a_{-}, \mathbf{c} ; v, \hat{v}\right)=R\left(a_{-}, a_{+}, I \hat{\mathbf{c}} ; \hat{v}, v\right)
\end{aligned}
$$

From these symmetry properties it is immediate that the $A \Delta O$ s (6.30) have eigenvalues (6.31) on $R$ iff one of the $\mathrm{A} \Delta$ Os yields the respective eigenvalue.

Even a sketch of the proof of the eigenfunction property would carry us too far afield-among other things, it involves various properties of the $G$ function [31]. To proceed, we detail one simple way to obtain the relativistic type II eigenfunction $R(x, p)$ : One can take

$$
R(x, p) \equiv R(2 \pi, \beta \nu,(\beta \nu g, \beta \nu g, 0,0) ; \nu x, \beta p) .
$$

Indeed, with this choice of variables the $\mathrm{A} \Delta \mathrm{Os} A_{+}(\mathbf{c} ; v)$ and $A_{+}(\hat{\mathbf{c}} ; \hat{v})$ turn into $H_{\text {rel }}^{t}(6.26)$ and $\widehat{H}_{\text {rel }}^{t}(6.27)$, respectively, and (6.28) and self-duality (symmetry under $\nu, \beta, x, p \rightarrow \beta, \nu, p, x$ ) follow by specialization from (6.30), (6.31), and (6.32), respectively.

As our final topic, we turn to relativistic type IV eigenfunctions, taking

$$
g=M+1=2,3,4, \ldots
$$

The $w$-function corresponding to this choice reads [31]

$$
w_{M}(x)=C s^{2}(x) \prod_{j=1}^{M} s(x+i j \beta) s(x-i j \beta), \quad C>0,
$$


and the corresponding $\mathrm{A} \Delta \mathrm{O} H_{\text {rel }}^{t}(6.25)$ will be written $A_{M}$. Anticipating the outcome of an Ansatz to be detailed shortly, we find eigenfunctions

$$
A_{M} R_{M}(x, \lambda)=E_{M}(\lambda) R_{M}(x, \lambda)
$$

depending on a complex parameter $\lambda$ that plays the role of $p$ for the previous case. (It does not reduce to $p$ for the hyperbolic specialization, however.) For an infinite sequence of $\lambda$-values the function $R_{M}(x, \lambda)$ is $2 \omega$ periodic or $2 \omega$-antiperiodic; taking $\beta(M+1) \in\left(0,-2 i \omega^{\prime}\right)$ (for simplicity), this countable infinity of functions spans a subspace $\mathcal{D}$ in the Hilbert space $\mathcal{H} \equiv L^{2}\left([0,2 \omega], w_{M}(x) d x\right)$ on which the $\mathrm{A} \Delta \mathrm{O} A_{M}$ is symmetric; in particular, the eigenvalues of $A_{M}$ on the sequence of functions are real. (Probably, $\mathcal{D}$ is dense in $\mathcal{H}$; if so, $A_{M}$ is Hilbert integrable.)

The function $R_{M}$ is of the form

$$
R_{M}(x, \lambda)=F_{M}(x, \lambda)+F_{M}(-x, \lambda)
$$

where $F_{M}( \pm x, \lambda)$ are linearly independent eigenfunctions with eigenvalue $E_{M}(\lambda)$. Since $A_{M}$ is parity-invariant, we need only discuss $F_{M}(x, \lambda)$. This eigenfunction is found via an Ansatz of the form

$$
\begin{aligned}
F_{M}(x, \lambda)=\left(\prod_{k=-M}^{M} s(x+i \beta k)\right)^{-1} & \prod_{j=1}^{M} s\left(x-z_{j}\right) \\
& \times \exp \left(\frac{i x}{2 \beta} \ln \left(\frac{s\left(z_{j}-i \beta\right)}{s\left(z_{j}+i \beta\right)}\right)\right)
\end{aligned}
$$

(This Ansatz was inspired by our previous results for the hyperbolic $g \in \mathbb{N}$ case [5].) Requiring that (6.34) with $R \rightarrow F$ hold true, one readily obtains

$$
E_{M}(\lambda)=C_{M}(x) \prod_{j=1}^{M}\left(s\left(i \beta+z_{j}\right) s\left(i \beta-z_{j}\right)\right)^{-1 / 2}
$$

where

$$
C_{M}(x) \equiv i^{M} \frac{s(x+i \beta M)}{s(x)} \prod_{j=1}^{M} \frac{s\left(x-i \beta-z_{j}\right)}{s\left(x-z_{j}\right)} s\left(i \beta-z_{j}\right)+(\beta \rightarrow-\beta) .
$$

Therefore, we obtain an eigenfunction whenever $C_{M}(x)$ does not depend on $x$.

We proceed by studying if and when the zeros $z_{1}, \ldots, z_{M}$ can be chosen such that this happens. We begin with the simplest case by far, namely, $M=1$. We take $z_{1}$ incongruent to 0 (modulo the period lattice), so that the poles at $x \equiv 0$ and $x \equiv z_{1}$ of the two summands of $C_{1}(x)$ are simple. Now $C_{1}(x)$ is elliptic in $x$, and since the residues at $x=0$ and $x=z_{1}$ 
cancel, $C_{1}(x)$ is constant. Thus we may put $x=i \beta$ to obtain (taking $\lambda$ equal to $z_{1}$ from now on)

$$
E_{1}(\lambda)=\frac{i s(2 i \beta)}{s(i \beta)^{2}}\left(\frac{1}{\mathcal{P}(\lambda)-\mathcal{P}(i \beta)}\right)^{1 / 2} .
$$

Summarizing, $F_{1}(x, \lambda)(6.35)$ is an eigenfunction of $A_{1}$ with eigenvalue $E_{1}(\lambda)(6.36)$. Letting the zero $z_{1}=\lambda$ vary over the line segment between $i \beta$ and $\omega^{\prime}$, the eigenvalue $E_{1}(\lambda)$ is positive and decreases monotonically from $\infty$ to a finite limit.

Next, we take $M>1$. Again, $C_{M}(x)$ is readily seen to be elliptic, but now $C_{M}(x)$ is not constant in general. However, we can proceed as follows. First, choose $z_{1}, \ldots, z_{M}$ pairwise incongruent, and incongruent to 0 . Then the two summands of $C_{M}(x)$ have only simple poles, and the residues at $x \equiv 0$ cancel. Requiring now that the residue sum at the poles $x \equiv z_{k}$ vanish, too, we obtain

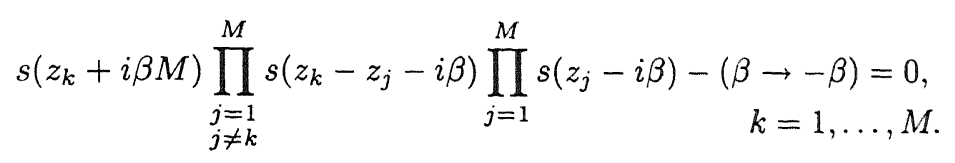

This constraint system of $M$ equations for $M$ unknowns $Z \equiv\left(z_{1}, \ldots, z_{M}\right)$ admits the solution

$$
Z_{0} \equiv(i \beta, 2 i \beta, \ldots, M i \beta)
$$

as is readily verified. An application of the implicit function theorem now shows that there exists a solution curve $\lambda \mapsto Z(\lambda)$ near $Z_{0}$, with $\lambda$ equal to $z_{1}$. Hence $C_{M}(x)$ is constant on the curve, and so we obtain eigenfunctions and eigenvalues depending on the curve parameter $\lambda$.

We intend to elaborate on the above assertions in Ref. 28. We conclude our sketch of type IV results by detailing the relation to the Lame functions that solve the eigenfunction problem for the operator $H_{\mathrm{nr}}(6.23)$, with the restriction (6.33) in force.

First, taking $\beta$ to 0 in (6.35) yields

$$
F_{M}(x, \lambda) \rightarrow s(x)^{-2 M-1} \prod_{j=1}^{M} s\left(x-z_{j}\right) \exp \left(\frac{x s^{\prime}\left(z_{j}\right)}{s\left(z_{j}\right)}\right) .
$$

Second, dividing the constraints (6.37) by $\beta$ and letting $\beta \rightarrow 0$, we obtain

$$
M \frac{s^{\prime}\left(z_{k}\right)}{s\left(z_{k}\right)}-\sum_{\substack{j=1 \\ j \neq k}}^{M} \frac{s^{\prime}\left(z_{k}-z_{j}\right)}{s\left(z_{k}-z_{j}\right)}-\sum_{j=1}^{M} \frac{s^{\prime}\left(z_{j}\right)}{s\left(z_{j}\right)}=0, \quad k=1, \ldots, M
$$


Third, recalling (2.50), we have

$$
\frac{s^{\prime}(x)}{s(x)}=\zeta(x)-\frac{\eta x}{\omega}
$$

where $\zeta$ is the Weierstrass $\zeta$-function. Therefore, the limit functions (6.38) and constraints (6.39) amount to the Lame functions and associated constraints that are specified by Whittaker and Watson; cf. p. 572 and p. 574 respectively, of Ref. 12. Quite recently, Etingof and Kirillov have tied in the latter functions with the representation theory of affine Lie algebras [20].

\section{REFERENCES}

1. M. A. Olshanetsky and A. M. Perelomov. Classical integrable finitedimensional systems related to Lie algebras. Phys. Rep., 71 (5): 313 $400,1981$.

2. M. A. Olshanetsky and A. M. Perelomov. Quantum integrable systems related to Lie algebras. Phys. Rep., 94 (6): 313-404, 1983.

3. S. N. M. Ruijsenaars and H. Schneider. A new class of integrable systems and its relation to solitons. Ann. Phys., 170 (2): 370-405, 1986.

4. S. N. M. Ruijsenaars. Complete integrability of relativistic CalogeroMoser systems and elliptic function identities. Commun. Math. Phys., 110 (2): 191-213, 1987.

5. S. N. M. Ruijsenaars. Finite-dimensional soliton systems. In B. Kupershmidt, ed., Integrable and Superintegrable Systems. World Scientific, Teaneck, NJ, pages 165-206, 1990.

6. J. F. van Diejen. Commuting difference operators with polynomial eigenfunctions. Comp. Math., 95 (2): 183-233, 1995.

7. J. F. van Diejen. Integrability of difference Calogero-Moser systems. J. Math. Phys., 35 (6): 2983-3004, 1994.

8. J. F. van Diejen. Difference Calogero-Moser systems and finite Toda chains. J. Math. Phys., 36 (3): 1299-1323, 1995.

9. V. I. Arnol'd. Mathematical Methods of Classical Mechanics, volume 60 of Graduate Texts in Mathematics. Springer-Verlag, New York, 1978.

10. W. Thirring. Lehrbuch der mathematischen Physik. I. Klassische dynamische Systeme. Springer-Verlag, Vienna, 1977. 
11. R. Abraham and J. E. Marsden. Foundations of Mechanics, 2nd edition. Benjamin-Cummings, Reading, MA, 1978.

12. E. T. Whittaker and G. N. Watson. A Course of Modern Analysis. Cambridge Univ. Press, Cambridge, 1973.

13. M. Reed and B. Simon. Methods of Modern Mathematical Physics III. Scattering Theory. Academic, New York, 1979.

14. B. Sutherland. Exact results for a quantum many-body problem in one dimension II. Phys. Rev., A5: 1372-1376, 1972.

15. G. J. Heckman. Root systems and hypergeometric functions II. Comp. Math., 64 (3): 353-373, 1987.

16. I. G. Macdonald. Orthogonal polynomials associated with root systems. preprint, 1988.

17. I. G. Macdonald. Orthogonal polynomials associated with root systems. In P. Nevai, ed., Orthogonal Polynomials, (Columbus, $\mathrm{OH}$, 1989), volume C294 of NATO ASI, 1990. Kluwer, Dordrecht, pages 311-318.

18. M. F. E. de Jeu. The Dunkl transform. Invent. Math., 113 (1): 147 $162,1993$.

19. P. I. Etingof and A. A. Kirillov, Jr. Macdonald's polynomials and representations of quantum groups. Math. Res. Lett., 1 (3): 279-296, 1994.

20. P. I. Etingof and A. A. Kirillov, Jr. Representations of affine Lie algebras, parabolic differential equations, and Lamé functions. Duke Math. J., 74 (3): 585-614, 1994.

21. M. Noumi. Macdonald's symmetric polynomials as zonal spherical functions on some quantum homogeneous spaces. Adv. Math., to appear.

22. I. Cherednik. Difference-elliptic operators and root systems. Internat. Math. Res. Notices, 1995 (1): 43-58, 1995.

23. R. Askey and J. Wilson. Some Basic Hypergeometric Orthogonal Polynomials that Generalize Jacobi Polynomials, volume 319 of Mem. Amer. Math. Soc. Amer. Math. Soc., Providence, RI, 1985.

24. R. Floreanini and L. Vinet. Quantum algebras and $q$-special functions. Ann. Phys., 221 (1): 53-70, 1993. 
25. T. H. Koornwinder. Orthogonal polynomials in connection with quantum groups. In P. Nevai, ed., Orthogonal Polynomials, (Columbus, $\mathrm{OH}, 1989$ ), volume C294 of NATO ASI, 1990. Kluwer, Dordrecht, pages 257-292.

26. T. H. Koornwinder. Askey-Wilson polynomials as zonal spherical functions on the SU(2) quantum group. SIAM J. Math. Anal., 24 (3): 795-813, 1993.

27. N. J. Vilenkin and A. U. Klimyk. Representation of Lie Groups and Special Functions III. Classical and Quantum Groups and Special Functions, volume 75 of Mathematics and Its Applications (Soviet Series). Kluwer, Dordrecht, 1992.

28. S. N. M. Ruijsenaars. Generalized Lamé functions. J. Math. Phys., to appear.

29. S. N. M. Ruijsenaars. Relativistic Calogero-Moser systems and solitons. In M. Ablowitz, B. Fuchssteiner, and M. Kruskal, eds., Topics in Soliton Theory and Exactly Solvable Nonlinear Equations, (Oberwolfach, 1986), 1987. World Scientific, Singapore, pages 182-190.

30. S. N. M. Ruijsenaars. Action-angle maps and scattering theory for some finite-dimensional integrable systems II. Solitons, antisolitons, and their bound states. Publ. RIMS, Kyoto Univ., 30 (6): 865-1008, 1994.

31. S. N. M. Ruijsenaars. First-order analytic difference equations and integrable quantum systems. J. Math. Phys., 38: 1069-1146, 1997.

32. G. Felder and A. P. Veselov. Shift operators for the quantum Calogero-Sutherland problems via the Knizhnik-Zamolodchikov equation. Commun. Math. Phys., 160 (2): 259-273, 1994.

33. J. Moser. Three integrable Hamiltonian systems connected with isospectral deformations. Adv. Math., 16: 197-220, 1975.

34. I. M. Krichever. Elliptic solutions of the Kadomtsev-Petviashvili equation and integrable systems of particles. Func. Anal. Appl., 14 (4): 282-290, 1980.

35. V. I. Inozemtsev. The finite Toda lattices. Commun. Math. Phys., 121 (4): 629-638, 1989.

36. B. Sutherland. An introduction to the Bethe ansatz. In B. S. Shastry, S. S. Jha, and V. Singh, eds., Exactly Solvable Problems in Condensed Matter and Relativistic Field Theory, (Panchgani, 1985), volume 242 of Lecture Notes in Physics, 1985. Springer-Verlag, Berlin, pages 1-95. 
37. S. N. M. Ruijsenaars. Relativistic Toda systems. Commun. Math. Phys., 133 (2): 217-247, 1990.

38. G. Frobenius. Über die elliptischen functionen zweiter art. J. Reine und Angew. Math., 93: 53-68, 1882.

39. A. K. Raina. An algebraic geometry study of the b-c system with arbitrary twist fields and arbitrary statistics. Commun. Math. Phys., 140 (2): 373-397, 1991.

40. S. N. M. Ruijsenaars. Action-angle maps and scattering theory for some finite-dimensional integrable systems III. Sutherland type systems and their duals. Publ. RIMS, Kyoto Univ., 31 (2): 247-353, 1995.

41. J. von Neumann. Mathematical Foundations of Quantum Mechanics. Princeton Univ. Press, Princeton, NJ, 1955.

42. M. Reed and B. Simon. Methods of Modern Mathematical Physics I. Functional Analysis. Academic, New York, 1972.

43. M. Reed and B. Simon. Methods of Modern Mathematical Physics II. Fourier Analysis, Self-Adjointness. Academic, New York, 1975.

44. M. Reed and B. Simon. Methods of Modern Mathematical Physics IV. Analysis of Operators. Academic, New York, 1978.

45. W. Thirring. Lehrbuch der mathematischen Physik. III. Quantenmechanik von Atomen und Molekülen. Springer-Verlag, Vienna, 1979.

46. E. M. Opdam. Root systems and hypergeometric functions III. Comp. Math., 67 (1): 21-49, 1988.

47. E. M. Opdam. Root systems and hypergeometric functions IV. Comp. Math., 67 (2): 191-209, 1988.

48. G. J. Heckman and E. M. Opdam. Root systems and hypergeometric functions I. Comp. Math., 64 (3): 329-352, 1987.

49. T. Oshima and H. Sekiguchi. Commuting families of differential operators invariant under the action of a Weyl group. J. Math. Sci. Univ. Tokyo, 2 (1): 1-75, 1995.

50. K. Sawada and T. Kotera. Integrability and a solution for the onedimensional $N$-particle system with inversely quadratic pair potentials. J. Phys. Soc. Japan, 39 (6): 1614-1618, 1975.

51. S. Wojciechowski. Involutive set of integrals for completely integrable many-body problems with pair interaction. Lett. Nuovo Cim., 18: $1 \cap 3-107 \quad 1977$ 
52. A. T. Fomenko. Integrability and Nonintegrability in Geometry and Mechanics, volume 31 of Mathematics and Its Applications (Soviet Series). Kluwer, Dordrecht, 1988.

53. S. N. M. Ruijsenaars. Action-angle maps and scattering theory for some finite-dimensional integrable systems I. The pure soliton case. Commun. Math. Phys., 115 (1): 127-165, 1988.

54. R. Goodman and N. R. Wallach. Classical and quantum-mechanical systems of Toda lattice-type III. Joint eigenfunctions of the quantized systems. Commun. Math. Phys., 105 (3): 473-509, 1986.

55. J. Dittrich and V. I. Inozemtsev. On the structure of eigenvectors of the multidimensional Lamé operator. J. Phys A: Math. Gen., 26 (16): L753-L756, 1993. 